Aus der Klinik für Neurologie und klinische Neurophysiologie (Chefarzt: Prof. Dr. med. F. Tergau) des St. Bernward Krankenhauses in Hildesheim

\title{
Sprachlateralisierung bei Epilepsiepatienten: Ein Vergleich der Ergebnisse funktioneller Magnetresonanztomografie (BOLD MRT) mit denen der Diffusionstensorbildgebung (DTI)
}

\author{
INAUGURAL-DISSERTATION \\ zur Erlangung des Doktorgrades \\ der Medizinischen Fakultät \\ der Georg-August-Universität zu Göttingen
}

vorgelegt von

Dorothee Bonnkirch (geb. Zingel)

aus

Meppen

Göttingen 2012 
Dekan:

I. Berichterstatter:

II. Berichterstatter:

III. Berichterstatter:

Tag der mündlichen Prüfung:
Prof. Dr. rer. nat. H. K. Kroemer

Prof. Dr. med. F. Tergau

Priv.-Doz. Dr. rer. nat. G. Helms

Prof. Dr. med. O. Gruber 


\section{INHALT}

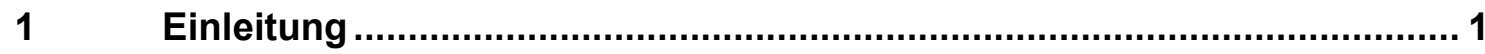

1.1 Einführung in Problemlage und Aufgabenstellung....................................... 1

1.2 Neuroanatomische Grundlagen der Sprache ............................................ 2

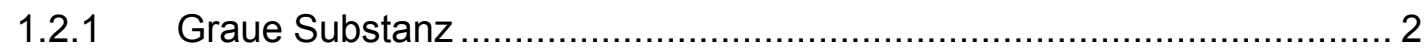

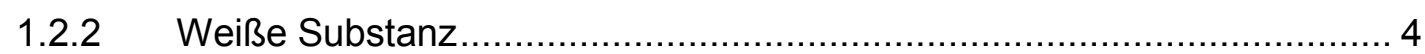

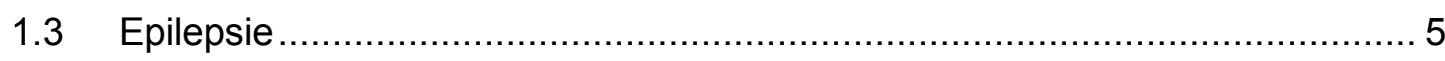

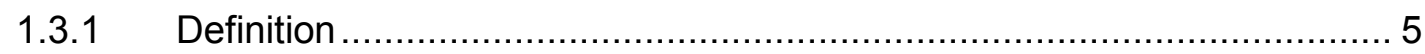

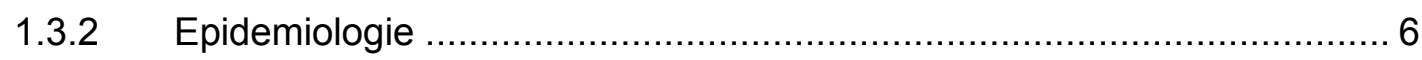

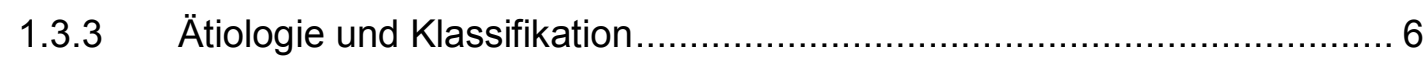

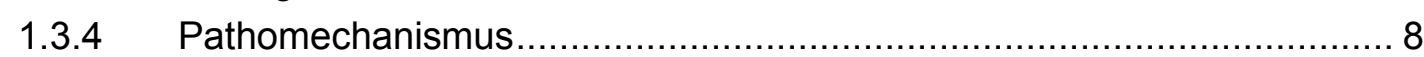

1.3.5 Krankheitsverlauf, Epilepsiediagnostik, Epilepsietherapie ...................... 9

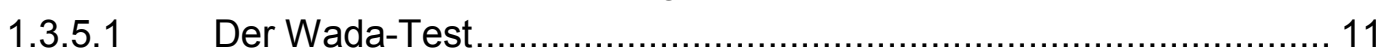

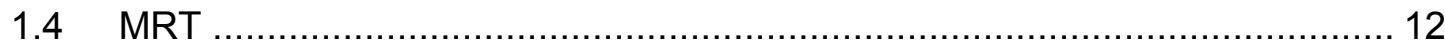

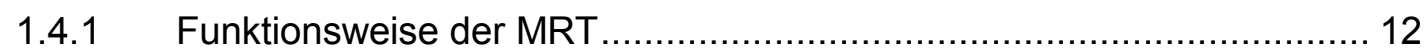

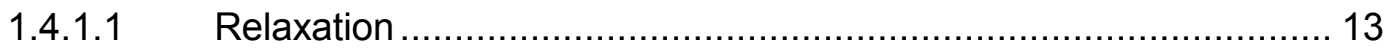

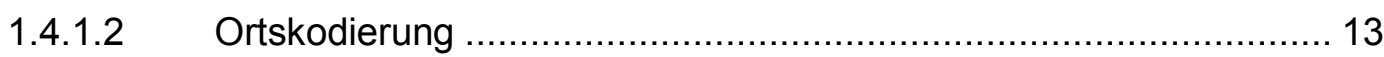

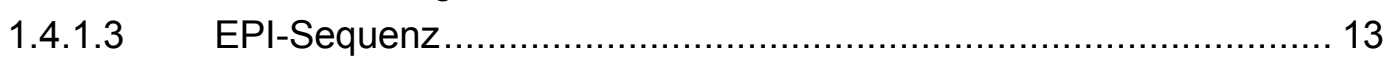

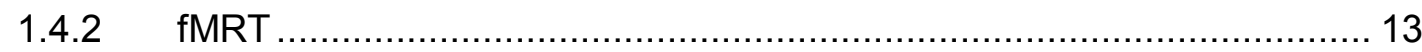

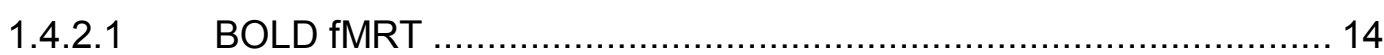

1.4.2.2 Die hämodynamische Antwortfunktion ........................................ 14

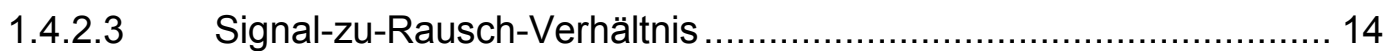

1.4.3 Auswertung von MRT-Daten …………………….............................. 15

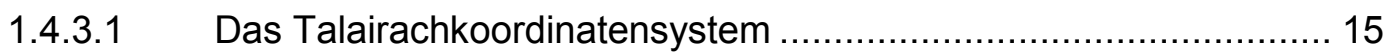

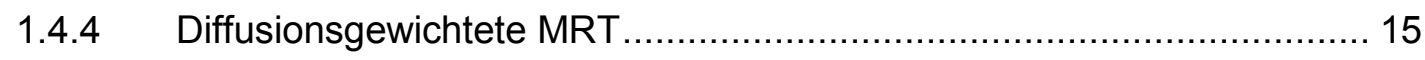

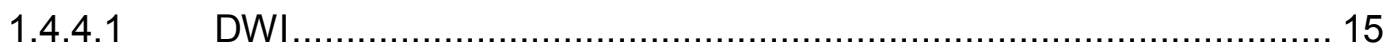

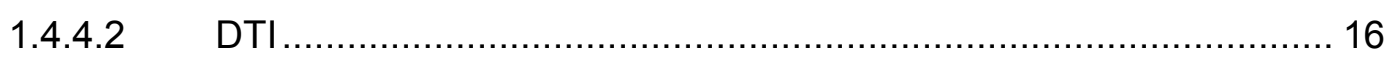

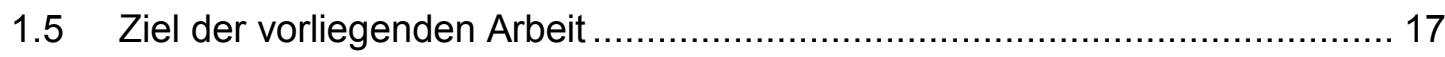

$2 \quad$ Material und Methode

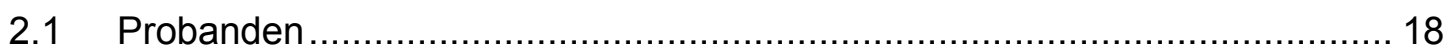

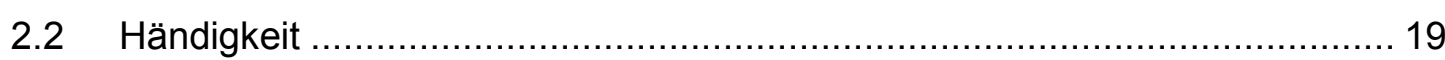

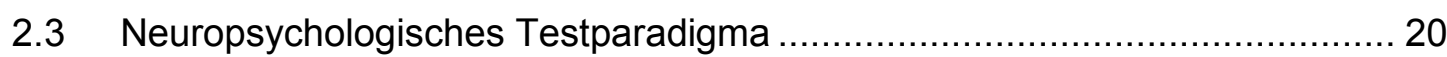

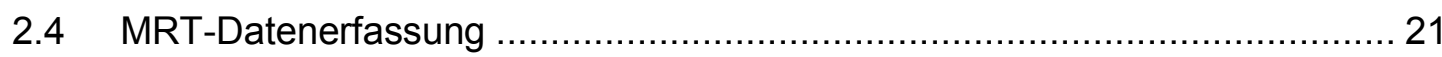

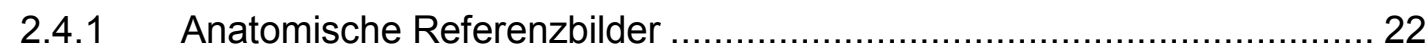

2.4.2 Funktionelle MRT-Bilder ................................................................. 22

2.4.3 Diffusionsgewichtete MRT-Bilder .................................................. 22

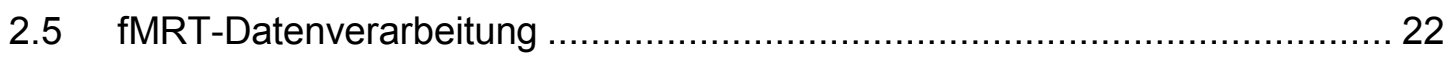

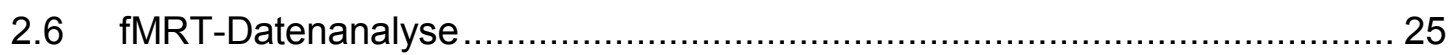




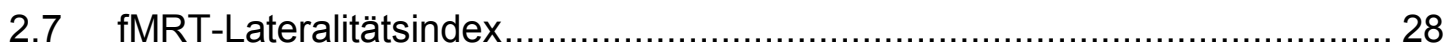

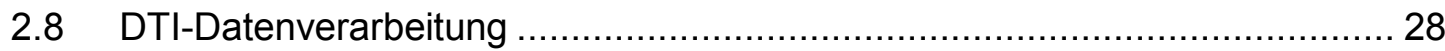

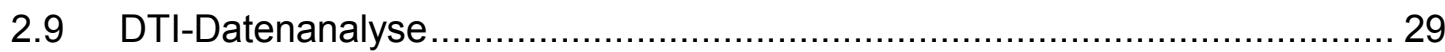

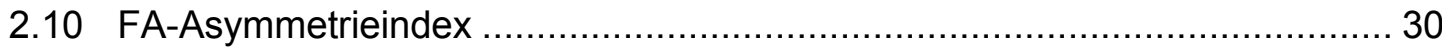

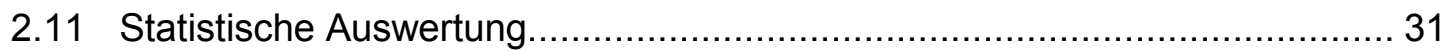

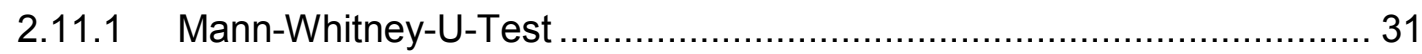

2.11.2 Vorzeichentest (nichtparametrisch) ............................................. 32

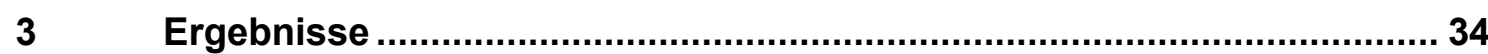

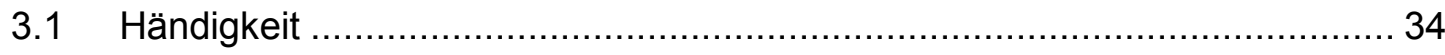

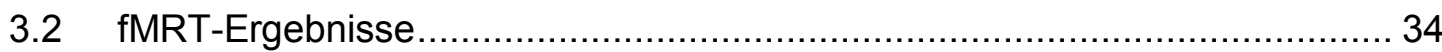

3.2.1 Sprachtest „Wortgenerierung“ in der Broca-Region ............................. 35

3.2.1.1 Broca-Region links im Kollektiv N........................................... 35

3.2.1.2 Broca-Region rechts im Kollektiv N ......................................... 35

3.2.1.3 Broca-Region links im Kollektiv E............................................... 36

3.2.1.4 Broca-Region rechts im Kollektiv E .......................................... 36

3.2.1.5 Vergleich der LIs in der Broca-Region während des Sprachtests

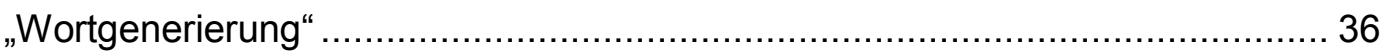

3.2.2 Sprachtest „Wortgenerierung“ in der Wernicke-Region ...................... 37

3.2.2.1 Wernicke-Region links im Kollektiv N ...................................... 38

3.2.2.2 Wernicke-Region rechts im Kollektiv N................................... 38

3.2.2.3 Wernicke-Region links im Kollektiv E ................................... 38

3.2.2.4 Wernicke-Region rechts im Kollektiv E................................... 39

3.2.2.5 Vergleich der LIs in der Wernicke-Region während des Sprachtests

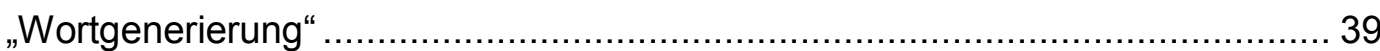

3.2.3 Sprachtest „Satzgenerierung“ in der Broca-Region ............................ 40

3.2.3.1 Broca-Region links im Kollektiv N............................................. 40

3.2.3.2 Broca-Region rechts im Kollektiv N ........................................... 41

3.2.3.3 Broca-Region links im Kollektiv E .......................................... 41

3.2.3.4 Broca-Region rechts im Kollektiv E ............................................ 42

3.2.3.5 Vergleich der LIs in der Broca-Region während des Sprachtests

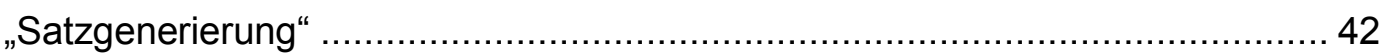

3.2.4 Sprachtest „Satzgenerierung“ in der Wernicke-Region........................ 43

3.2.4.1 Wernicke-Region links im Kollektiv N .......................................... 43

3.2.4.2 Wernicke-Region rechts im Kollektiv N.................................... 43

3.2.4.3 Wernicke-Region links im Kollektiv E ........................................... 44

3.2.4.4 Wernicke-Region rechts im Kollektiv E...................................... 44

3.2.4.5 Vergleich der LIs in der Wernicke-Region während des Sprachtests

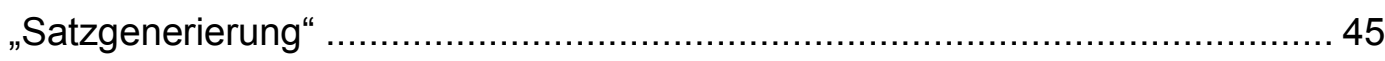

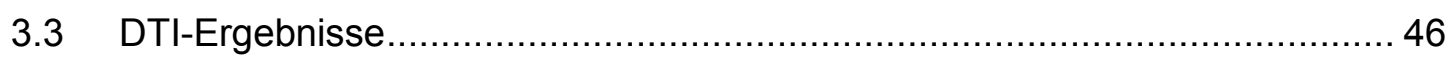

3.3.1 DTI-Auswertung der Broca-Region.................................................. 46

3.3.1.1 Asymmetrieindizes der strukturellen ROI „Wort Broca“.................. 46 
3.3.1.2 Vergleich der Als in der strukturellen ROI „Wort Broca“................. 47

3.3.1.3 Asymmetrieindizes der strukturellen ROI „Satz Broca“.................. 47

3.3.1.4 Vergleich der Als in der strukturellen ROI „Satz Broca“................. 48

3.3.2 Asymmetrieindizes in der Wernicke-Region .................................. 48

3.3.2.1 Asymmetrieindizes der strukturellen ROI „Wort Wernicke“............. 48

3.3.2.2 Vergleich der Als in der strukturellen ROI „Wort Wernicke“............ 49

3.3.2.3 Asymmetrieindizes der strukturellen ROI „Satz Wernicke“ ............. 49

3.3.2.4 Vergleich der Als in der strukturellen ROI „Satz Wernicke“ ............ 50

3.4 Kombinierte Analyse von fMRT und DTI ............................................... 50

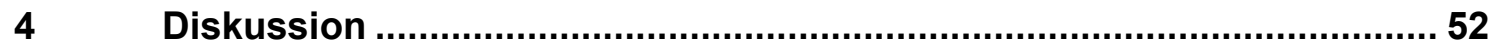

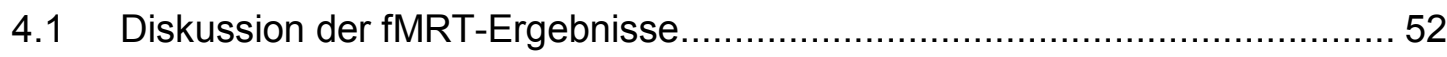

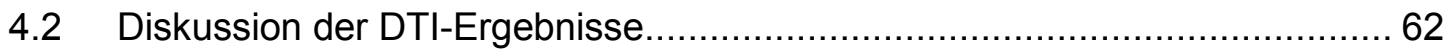

4.3 Abschließende Bewertung und Ausblick ............................................. 70

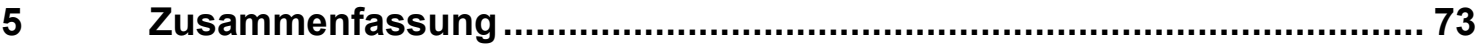

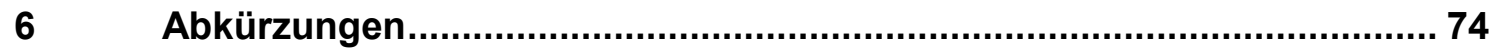

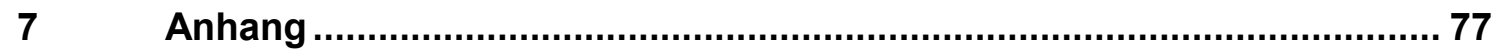

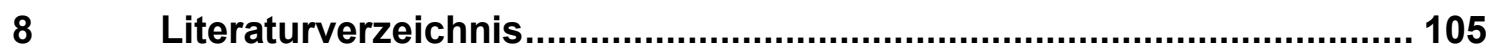




\section{EINLEITUNG}

\subsection{Einführung in Problemlage und Aufgabenstellung}

Kognitive Funktionen, wie z. B. Sprache, sind in den beiden Hirnhälften unterschiedlich ausgeprägt, wobei eine Hemisphäre sprachdominant ist. Bei Patienten mit langjähriger Temporallappenepilepsie weicht die Lokalisation der sprachrelevanten Kortexareale häufig von der typischen Lokalisation gesunder Probanden ab (Adcock et al. 2003; Springer et al. 1999). Klinisch relevant ist die exakte Seitenbestimmung der sprachrelevanten Areale in der Diagnostik von Patienten mit Temporallappenepilepsie, bei denen ein epilepsiechirurgischer Eingriff geplant ist. Nur durch eine exakte Bestimmung der Sprachlateralität kann das Verletzungsrisiko sprachrelevanter Strukturen während der Operation eingeschätzt und das Risiko postoperativer Sprachdefizite minimiert werden.

Der Gold-Standard zur Bestimmung der Sprachlateralisierung ist die kathetergestützte selektive Narkotisierung einer Hemisphäre im Rahmen des Wada-Tests. Der WadaTest ist sehr invasiv und stellt eine hohe Belastung für den Patienten dar. Alternativ kann die Sprachlateralisierung mit nichtinvasiven bildgebenden Verfahren, wie funktionelle Magnetresonanztomografie (fMRT) und Diffusions-Tensor-Bildgebung (DTI), untersucht werden (Detre 2004; Sundgren et al. 2004).

Darüber hinaus könnten fMRT-gestützte Verfahren in Kombination mit anatomischen Daten präzise Angaben über die Lokalisation von Kortexarealen machen. Auf diese Weise könnten sie zukünftig für die Planung von Resektionsgrenzen in der Epilepsiechirurgie von großem Nutzen sein. Eine Voraussetzung dafür ist jedoch die verlässliche Identifizierung der sprachrelevanten Kortexareale.

In der vorliegenden Arbeit wurde untersucht, ob die DTI-basierte Bildgebung eine pathologisch bedingte atypische Sprachlateralisierung bei Patienten mit fokaler Epilepsie darstellen kann und die nichtinvasive Diagnostik mittels fMRT dadurch sinnvoll ergänzt werden kann. Die strukturellen Informationen der DTI-Bildgebung wurden mit den fMRT-Ergebnissen verglichen und auf eine Assoziation zwischen struktureller Asymmetrie und funktioneller Sprachlateralisierung geprüft. Durch die Kombination dieser beiden Magnetresonanzverfahren sollte eine verlässliche Methode zur Lokalisation sprachrelevanter Hirnregionen etabliert werden, die eine praktikable Alternative zum Wada-Test darstellen könnte.

Die Fragestellung wurde durch den Vergleich eines Patientenkollektivs mit fokaler Epilepsie und einem altersentsprechenden Kollektiv gesunder Versuchspersonen bearbeitet. Bei allen Patienten mit fokaler Epilepsie wurde im Vorfeld der Studie durch 
eine Video-Elektroenzephalogramm-Untersuchung die Seitenlokalisation der Epileptogenese sicher bestimmt. Als Stimulationsparadigmen während der funktionellen Bildgebung wurden etablierte Sprachtests der neuropsychologischen Magnetresonanzforschung angewendet.

\subsection{Neuroanatomische Grundlagen der Sprache}

Die Neuroanatomie der Sprache besteht aus verschiedenen sprachassoziierten Kortexarealen der grauen Substanz und Assoziationsfasern der weißen Substanz.

\subsubsection{Graue Substanz}

Die primären sprachassoziierten Kortexareale können durch ein makroskopisches und ein mikroskopisches Konzept beschrieben werden. In der makroskopischen Einteilung stehen das Broca- und das Wernicke-Sprachzentrum im Vordergrund, während die mikroskopische Betrachtungsweise auf der Zytoarchitektur des Neokortex und seiner Einteilung in Brodmann-Areale (BA) beruht.

Die makroskopische Einteilung der beiden wichtigsten die Sprache repräsentierenden Kortexareale in das motorische Broca-Sprachzentrum und das sensorische WernickeSprachzentrum ist historisch und beruht auf Fallstudien:

Der französischer Anthropologe und Arzt Pierre Paul Broca (1824-1880) untersuchte postmortale Hirnläsionen bei Patienten mit Aphasie, d.h. der Unfähigkeit, Sprache zu produzieren oder zu verstehen. Er beschrieb erstmals den Zusammenhang zwischen Aphasie und einer Hirnläsion im linken vorderen unteren Frontallappen. Nach inm ist das motorische Broca-Sprachzentrum benannt, welches die Pars opercularis und zum Teil die Pars triangularis des Gyrus frontalis inferior umfasst. Neuere Erkenntnisse lassen vermuten, dass auch umliegende Regionen des Gyrus frontalis inferior zum motorischen Sprachzentrum gehören (Bookheimer 2002). Broca begründete außerdem das Konzept der Sprachdominanz. Er entdeckte, dass das motorische Sprachzentrum nur in einer Hemisphäre seine Funktion ausübt, und erklärte diese Hemisphäre für dominant. In der Broca-Region wird die Sprache in Wort und Satzbau geformt. Das motorische Sprachzentrum erhält afferente Informationen von der primären und sekundären Hörrinde, von Assoziationsfeldern und vom Gyrus angularis. Die Efferenzen der Broca-Region gelangen direkt oder über Verschaltung mit den Basalganglien, dem Kleinhirn und dem Thalamus zum Gyrus praecentralis, der die Impulse an die lautbildenden Muskeln weiterleitet.

Der deutsche Neurologe und Psychiater Carl Wernicke (1848-1904) entdeckte, dass neben dem Broca-Areal noch ein weiteres Sprachzentrum existiert: das sensorische Wernicke-Sprachzentrum. Dieses Zentrum ist ebenfalls in der sprachdominanten 
Hemisphäre lokalisiert und umfasst den hinteren Anteil des Gyrus temporalis superior, einen Bestandteil der sekundären Hörrinde. In der Wernicke-Region wird das Sprachverständnis durch Erkennung und Interpretation von auditorischen Impulsen ausgebildet. Dazu erhält das sensorische Sprachzentrum afferente Informationen von der primären Hörrinde und dem Gyrus angularis. Die primäre Hörrinde verarbeitet das Bewusstwerden von Sprache, während der Gyrus angularis Impulse aus dem sekundär visuellen Kortex erhält und für die Zuordnung sprachlicher Begriffe zu visuellen Eindrücken zuständig ist (Dronkers et al. 2000).

Als sprachdominante Hemisphäre wird diejenige Hemisphäre bezeichnet, in der die motorische und sensorische Sprachverarbeitung stattfindet. Bei Rechtshändern ist dieses in der Regel die linke Hemisphäre, während bei Linkshändern häufiger die rechte Hemisphäre dominant ist oder die Sprachverarbeitung gleichmäßig in beiden Hemisphären stattfindet (Lurito und Dzemidzic 2001).

Die mikroskopische Einteilung der sprachassoziierten Kortexareale geht auf den deutschen Neuroanatom und Psychiater Korbinian Brodmann (1868 - 1918) zurück. In histologischen Schnitten entdeckte er Unterschiede im Schichtaufbau des Neokortex, die die Grundlage für die Einteilung des Neokortex in 52 zytoarchitektonische Felder, die Brodmann-Areale (BA), bilden (Brodmann 1909) (Abbildung 1).

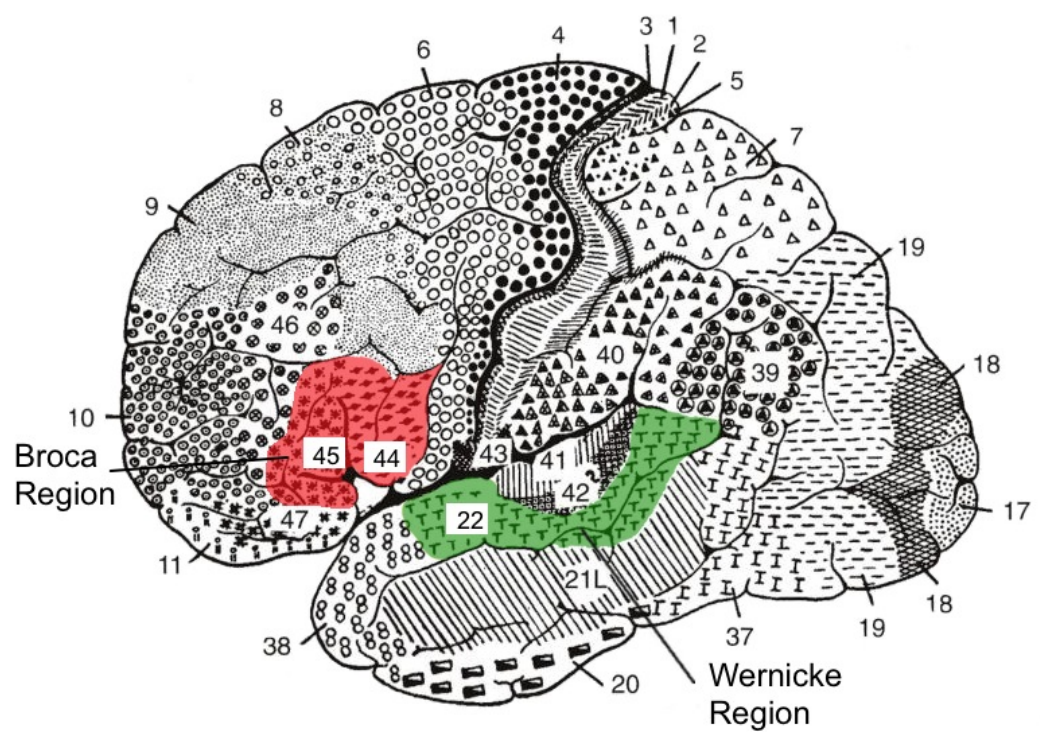

Abbildung 1: Zytoarchitektonische Gliederung des Kortex nach Brodmann (Brodmann 1909, S. 131). BA 44 und 45 entsprechen der Broca-Region (rot), BA 22 entspricht der Wernicke-Region (grün).

Eine Zuordnung zytoarchitektonisch definierter BA zu den makroskopisch definierten Broca- und Wernicke-Sprachzentren ist aufgrund der großen interindividuellen und interhemisphärischen Variabilität der Ausdehnung von BA innerhalb von Gyri und Sulci nur ungefähr möglich. Da sich die Grenzen der zytoarchitektonischen Areale nicht an die Grenzen der Gyri und Sulci halten und die BA und Sulcusmuster unabhängig voneinander variieren, geben Sulci, die die makroskopischen Grenzen der Broca- und 
Wernicke-Region bilden, nur eine ungefähre Orientierungshilfe für die Lage der BA innerhalb dieser Sprachregionen.

Die Zuordnung von BA zur Broca- und Wernicke-Region ist Gegenstand intensiver wissenschaftlicher Forschungen. Konsens besteht darüber, dass die Broca-Region dem BA 44 und BA 45 entspricht (Aboitiz und Garcia 1997). Eventuell kann auch BA 47 der Broca-Region zugeordnet werden (Amunts et al. 1999). Bei der Zuordnung des BA 22 zur Wernicke-Region herrscht Übereinstimmung. Eventuell gehören auch das BA 40, BA 39 und BA 37 funktionell zur Wernicke-Region (Aboitiz und Garcia 1997) (Abbildung 1).

Es existieren weitere sprachassoziierte Areale, auf die an dieser Stelle jedoch nicht eingegangen wird, da nur die primären Sprachareale für das Verständnis der vorliegenden Arbeit von Bedeutung sind.

Die exakte Lokalisation der sprachrelevanten Kortexareale in vivo ist aufgrund der beschriebenen individuellen Ausprägung der BA äußerst schwierig. Eine verlässliche diagnostische Methode ist für die Planung epilepsiechirurgischer Eingriffe jedoch von größter Bedeutung. Diese Arbeit versucht ein standardisiertes und zuverlässiges bildgebendes Verfahren zur Lokalisation sprachrelevanter Kortexareale zu entwickeln.

\subsubsection{Weiße Substanz}

Die weiße Substanz des zentralen Nervensystems (ZNS) wird der grauen Substanz gegenübergestellt und besteht aus Nervenfasern und neuronalem Bindegewebe (Gliazellen etc.). Je nach ihrem Verlauf können die Faserbahnsysteme der weißen Substanz in drei verschiedene Kategorien eingeteilt werden: Kommissurenfasern verbinden beide Hemisphären miteinander, Projektionsfasern verbinden die Hirnrinde mit subkortikalen Kernen und Assoziationsfasern verbinden unterschiedliche Areale einer Hemisphäre miteinander.

Norman Geschwind (1926-1984), ein US-amerikanischer Neurologe, untersuchte Prinzipien der Sprachverarbeitung und etablierte das Wernicke-Geschwind-Modell (Geschwind 1970). Anhand von Untersuchungen an aphasischen Patienten erweiterte Geschwind die Konzepte von Broca und Wernicke und fasste die Ergebnisse in einem vereinfachten Modell zur Sprachverarbeitung zusammen, das immer noch als Grundlage für neuere Modelle gilt.

Geschwind (1970) postulierte, dass neben der Broca- und der Wernicke-Region auch Strukturen der weißen Substanz eine essentielle Bedeutung in der Sprachverarbeitung besitzen. So verbindet z. B. der Fasciculus arcuatus, ein Assoziationsbündel der weißen Substanz, bidirektional das Wernicke-Sprachzentrum mit dem BrocaSprachzentrum. Auf diese Weise sind Sprachbildung und Sprachverständnis miteinander assoziiert (Abbildung 2). 


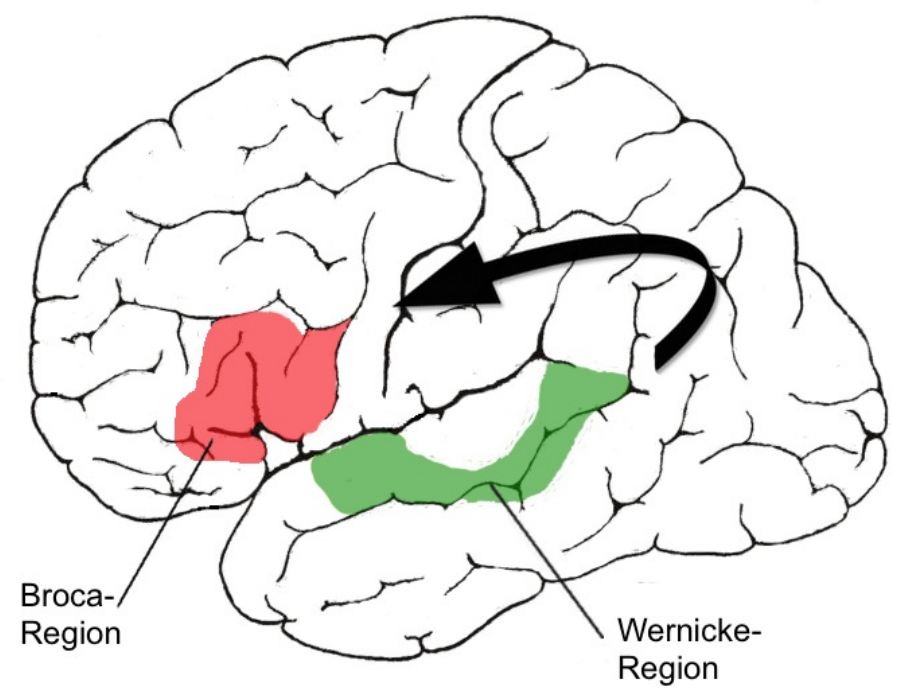

Abbildung 2: Schematische Darstellung der an der kognitiven Funktion Sprache beteiligten Strukturen. Der Fasciculus arcuatus (schwarzer Pfeil) bildet eine Verbindung zwischen der Broca- (rot) und der Wernicke-Region (grün).

\subsection{Epilepsie}

\subsubsection{DEFINITION}

Die Epilepsie ist eine der häufigsten neurologischen Erkrankungen weltweit. Ein epileptischer Anfall zeichnet sich durch das vorübergehende Auftreten abnorm exzessiver und synchroner neuronaler Aktivität des ZNS aus, welches sich je nach betroffenem Hirnareal in verschiedenen klinischen Symptomen äußert. Es kommt zu veränderter sensorischer, motorischer und autonomer Funktion, Veränderungen in Bewusstsein, Emotionen, Gedächtnis, Kognition und Verhalten. Diese Symptome können einzeln oder zusammen auftreten.

Kommt es zu rezidivierenden epileptischen Anfällen, besteht eine anhaltende erhöhte Prädisposition, epileptische Anfälle zu erleiden und es liegt eine Epilepsie vor. Die Ursachen einer Epilepsie können dabei sehr unterschiedlich sein. Eine Epilepsie hat weitreichende sozialpsychologische Konsequenzen für die Patienten und ihre Familien, denn sie führt zu Verhaltensveränderungen, häufig zu kognitiven Defiziten, Stigmatisierung, Ausschluss, Einschränkungen und Isolation. 


\subsubsection{EPIDEMIOLOGIE}

$5 \%$ der Bevölkerung erleiden während ihres Lebens einen Gelegenheitsanfall, der jedoch laut Definition der International League Against Epilepsy (ILAE) von einer Epilepsie zu differenzieren ist (Fisher et al. 2005).

Die Prävalenz der Epilepsie entspricht $0,5-1 \%$ der Bevölkerung. Die jährliche Inzidenz der Epilepsie, d.h. die Anzahl der Neuerkrankungen in einem Jahr, beträgt 50 pro 100.000 Einwohner, wobei die Erstmanifestation am häufigsten im Kindesalter sowie nach dem 60. Lebensjahr auftritt (Hufschmidt et al. 2009).

Da nicht alle Epilepsiepatienten auf eine medikamentöse Therapie ausreichend ansprechen, kann bei einer fokalen Epilepsie (s.u.) ein neurochirurgischer Eingriff in Betracht gezogen werden. Die Inzidenz der Epilepsiepatienten, welche die Kriterien für eine epilepsiechirurgische Maßnahme erfüllen, beläuft sich in Deutschland auf 200 bis 1.000 Patienten pro Jahr (May und Pfäffin 2000).

\subsection{3 Ätiologie UND KLASSIFIKATION}

Epilepsien werden je nach Ursprungsort und Ausbreitungsform der abnormen neuronalen Aktivität in fokale oder generalisierte Epilepsien eingeteilt.

$60 \%$ der Epilepsien sind fokale Epilepsien. Diese sind durch Anfälle charakterisiert, die einen fokalen Beginn haben und auf Teile des Gehirns beschränkt bleiben und somit nicht generalisiert sind. Die verbleibenden 40\% machen generalisierte Epilepsie aus (Hauser et al. 1991). Für die verschiedenen Epilepsieformen gelten altersspezifische Prävalenzen. So treten generalisierte Epilepsien vermehrt im Kleinkindalter auf, fokale dagegen eher im Erwachsenenalter. Eine der häufigsten fokalen Epilepsien im Erwachsenenalter, die als Syndrom gut charakterisiert ist, ist die Temporallappenepilepsie (Engel 2001).

Fokale Anfälle können, abhängig von der Bewusstseinslage des Patienten während eines epileptischen Anfalls und dem klinischen Anfallsverlauf, in einfach-fokale, komplex-fokale und sekundär generalisierte Anfälle unterschieden werden, die ineinander übergehen können.

Komplex-fokale Anfälle gehen immer mit einer Bewusstseinsveränderung einher; bei einfach-fokalen Anfällen liegt dagegen keine Bewusstseinsstörung vor. Bewusstseinsstörungen sind alle Zustände eines Patienten, in denen dieser vermindert kontakt- oder reaktionsfähig ist (Stefan 1995). Sekundär generalisierte Anfälle beginnen fokal, gehen jedoch im Verlauf in eine bilaterale tonisch-klonische Phase über.

Hinsichtlich ihrer Ätiologie können Epilepsien bislang in idiopathische, symptomatische oder kryptogene Epilepsien unterschieden werden. Viele idiopathische Epilepsien 
zeichnen sich durch eine genetische Disposition aus mit polygenem Erbgang und multifaktorieller Genese. Symptomatischen Epilepsien hingegen liegt ein klar zu diagnostizierender, meist nicht genetischer Fokus zugrunde, von dem die epileptischen Anfälle ausgehen. Dazu zählen z.B. erworbene Hirnläsionen aufgrund eines Tumors, eines Traumas, eines entzündlichen Prozesses oder einer Ischämie. Auch Fehlbildungen bzw. Entwicklungsstörungen des Gehirns oder neuronale Migrationsstörungen in der Embryonalphase, wie z.B. fokale Dysplasien oder Heterotopien, zählen zur Ätiologie symptomatischer Epilepsien. Als kryptogen werden hingegen alle Epilepsien bezeichnet, denen (noch) keine zugrunde liegende Ursache nachgewiesen werden kann, jedoch eine symptomatische Ursache angenommen werden muss.

Aufgrund des Fortschritts der neurologischen Diagnostik und der Bildgebung sowie der daraus gewonnenen Erkenntnisse über die Entstehung von Epilepsien wird gegenwärtig die Überarbeitung dieser Klassifikation diskutiert. Eine von 2009 stammende Einteilung der ILEA schlägt eine Klassifikation der Epilepsien auf Grundlage ihrer Ätiologie in genetisch, strukturell/metabolisch und unbekannter Ursache vor (Berg et al. 2010). Genetische Epilepsien sind in dieser Einteilung die direkte Folge von bekannten oder angenommenen genetischen Defekten, die als Hauptsymptom epileptische Anfälle bedingen. Strukturellen/metabolischen Epilepsien liegen andere Zustände oder Erkrankungen zugrunde, die mit einem erhöhten Risiko für die Entstehung einer Epilepsie assoziiert sind. Dazu gehören auch erworbene Hirnläsionen wie Schlaganfall, Trauma oder Infektion und genetisch bedingte Malformationen wie die Hippokampussklerose. Bei Epilepsien mit unbekannter Ursache liegt möglicherweise ein genetischer Defekt oder eine andere Erkrankung, die bisher noch nicht entdeckt wurde, zugrunde.

Da bei fokalen Epilepsien die paroxysmalen synchronen Entladungen immer an einem umschriebenen Ort (epileptogener Fokus) entstehen, können sie nach der Fokuslokalisation in frontale, temporale, okzipitale oder parietale fokale Epilepsien eingeteilt werden. Ziel der Epilepsiediagnostik ist immer auch die genaue räumliche Zuordnung der Epileptogenese. Dabei ist der elektroenzephalographische Fokus unabhängig von der Identifikation einer strukturellen Läsion an diesem Fokus.

Die exakte Klassifikation einer Epilepsie ist entscheidend für die Prognose und Therapie des Patienten. So sprechen generalisierte und fokale Epilepsien in der Regel auf Medikamente unterschiedlich gut an und die Option eines kurativen epilepsiechirurgischen Eingriffs ergibt sich nur bei fokalen Epilepsien.

Diese Arbeit betrachtet ausschließlich Patienten mit einer gesicherten fokalen Epilepsie strukturell/metabolischer oder unbekannter Ursache und damit einhergehenden charakteristischen Anfällen. Diese Patientengruppe stellt den größten Anteil der erwachsenen Patienten, die an Epilepsie leiden, dar. 


\subsubsection{Pathomechanismus}

Der Pathomechanismus von Epilepsien ist noch nicht in jeglicher Hinsicht geklärt. Soweit für die Bearbeitung der Fragestellung dieser Arbeit notwendig, werden die zum aktuellen Stand der Forschung bekannten Entstehungsfaktoren epileptischer Anfälle hier vorgestellt.

Epileptische Anfälle entstehen aufgrund einer Störung des Gleichgewichts zwischen Exzitation und Inhibition von Neuronen, welche zu exzessiven neuronalen Entladungen im ZNS führt. Geschieht dieses in einem Areal oder mehreren umschriebenen Arealen (Fokus), folgt ein fokaler epileptischer Anfall (Stefan 1995).

Neurone eines epileptischen Fokus zeigen eine von gesunden Neuronen abweichende Funktion, die sich interiktal vom Zellinneren ableiten lässt. Die Depolarisation geht mit einer hochfrequenten Serie von Aktionspotenzialen einher. Dieses Phänomen führt zu einer übermäßig langen Depolarisation im Vergleich zu einem gesunden Neuron. Die Depolarisation wird durch eine Hyperpolarisation beendet. Dieses Phänomen des „Paroxysmalen Depolarisationsshift“ (PDS) wurde zuerst von Matsumoto und AjmoneMarsan (1964) beschrieben. Es existieren jedoch Regulationsmechanismen, welche die Ausbreitung der Erregung inhibieren und einen epileptischen Anfall verhindern. Zum einen besteht um den Fokus herum ein konzentrischer Hemmungshof (surround inhibition) (Prince und Wilder 1967), zum anderen tritt eine vertikale Inhibition auf (Elger und Speckmann 1983), die die Ausbreitung in die Tiefe des Gehirns unterbindet.

Aktuell existieren mehrere Hypothesen zur Epileptogenese fokaler Epilepsien. Auf molekularer Ebene kommt dem $\mathrm{GABA}_{\mathrm{A}}$-Rezeptor (GABA: Gamma-Aminobuttersäure) eine große Bedeutung zu. So könnten verminderte Synthese und Wirkung des inhibitorischen Transmitters GABA an den Zellen eines epileptischen Fokus sowie eine Verstärkung der Depolarisation durch die exzitatorische Aminosäure Glutaminsäure eine Ursache darstellen (Avoli und Gloor 1987).

Bei der Hippokampussklerose, einer häufigen Ursache einer Temporallappenepilepsie (Mathern et al. 1997), spielen vor allem morphologische Veränderungen eine Rolle. Durch strukturelle Reorganisation infolge der Hippokampussklerose könnten rekurrente Erregungskreise entstehen (Okazaki et al. 1995). Des Weitern könnte ein selektiver Verlust von Interneuronen zu einer Hyperexzitation im Temporallappen führen (Sloviter 1987). Auch die Neurogenese von Progenitor-Zellen im Hippokampus mit fehlerhafter Integration könnte in einem Ungleichgewicht zwischen Exzitation und Inhibition resultieren (Scharfman et al. 2000).

Diese morphologischen Vorgänge können zu fokaler Veränderung des Hirnaufbaus mit Funktionseinbußen der betroffenen Kortexareale führen, deren neurophysiologische Dokumentation möglich ist (Helmstaedter et al. 2009). Im Zuge der Neuroplastizität 
besteht die Möglichkeit, dass andere Hirnareale die Funktion der geschädigten Kortexareale übernehmen.

Neuroplastizität ist ein Prozess, bei dem sich synaptische Systeme durch molekulare und zelluläre Faktoren verändern, um die Kommunikationseffizienz neuronaler Netzwerke zu verbessern (Demonet et al. 2005). Der Mechanismus der Neuroplastizität ermöglicht eine postläsionale Erholung von Hirnfunktionen wie z. B. der Sprache. Dabei übernehmen Kortexareale, die ursprünglich keine Funktion für die beeinträchtigte kognitive Fähigkeit besaßen, die funktionellen Aufgaben geschädigter Hirnareale. Auch epileptische Dysfunktionen können auf diese Weise eine Verschiebung sprachrelevanter Kortexareale bewirken (Helmstaedter et al. 1997).

Da bei Rechtshändern in der Regel der linke Temporallappen in die Sprache involviert ist, zeigen insbesondere Patienten mit chronischer Temporallappenepilepsie eine Reorganisation von temporalen Sprachnetzwerken (Breier et al. 2005; Pataraia et al. 2004). Die Reorganisation von Sprache kann dabei auf zwei Arten geschehen. Bei der intrahemisphärischen Reorganisation übernehmen nicht betroffene Kortexareale der Hemisphäre des epileptogenen Fokus die Aufgaben der sprachrelevanten Kortexareale. Bei der interhemisphärischen Reorganisation übernehmen dagegen homologe Kortexareale der kontralateralen Hemisphäre die Aufgaben der geschädigten Sprachregionen (Bell et al. 2002; Breier et al. 2005; Muller et al. 1999; Pataraia et al. 2004). Aufgrund dieser Mechanismen kann es bei Patienten mit Temporallappenepilepsie zu einer atypischen Repräsentation der Sprache mit bilateraler oder rechtsseitiger Hemisphärendominanz kommen (Adcock et al. 2003). Auch Cousin et al. (2008) konnten nachweisen, dass Patienten mit einer Temporallappenepilepsie und ursprünglicher linkshemisphärischer Sprachdominanz ein geringeres Maß an Hemisphärenlateralisierung sowie eine zusätzliche Beteiligung der rechten Hemisphäre zeigen. Die zerebrale Reorganisation bei Patienten mit Temporallappenepilepsie ist unter anderem vom Alter während des Krankheitsbeginns und dem Vorliegen einer Hippokampussklerose abhängig. Bei frühem Krankheitsbeginn liegt eine erhöhte Tendenz zur intra- und interhemisphärischen Reorganisation vor. Außerdem fördert das Vorliegen einer Hippokampussklerose die interhemisphärische Verschiebung temporaler Sprachaktivierung (Bell et al. 2002; Muller et al. 1999).

Diese atypische Sprachrepräsentation ist auch mit den in der vorliegenden Arbeit verwendeten bildgebenden Verfahren nachweisbar und zeigt die Notwendigkeit einer verlässlichen Lokalisation der individuell ausgeprägten sprachrelevanten Kortexareale.

\subsubsection{KRANKHEITSVERLAUF, EPILEPSIEDIAGNOSTIK, EPILEPSIETHERAPIE}

Bei Auftreten eines ersten epileptischen Anfalls wird zunächst untersucht, ob dieser epileptische Anfall ein durch äußere Faktoren wie Schlafmangel oder Alkohol 
provozierter Gelegenheitsanfall ist, oder ob eine behandlungsbedürftige Ursache dem Anfall zugrunde liegt. Außerdem wird untersucht, ob der erste Anfall bereits den Beginn einer Epilepsie darstellt und einem Epilepsiesyndrom zugeordnet werden kann.

Da ein epileptischer Anfall das Symptom einer strukturellen neurologischen Erkrankung sein kann, wird in der Epilepsiediagnostik eine MR-Aufnahme erstellt. Diese kann strukturelle Hirnveränderungen, wie z.B. einen raumfordernden Prozess oder eine Ammonshornsklerose, zeigen. Außerdem ist das Elektroenzephalogramm (EEG) essentieller Bestandteil der Epilepsiediagnostik. Das EEG kann auch interiktal pathologische Aktivität in Neuronenverbänden zeigen und eventuell schon eine Zuordnung zu einem Epilepsiesyndrom ermöglichen. Eine besondere Bedeutung kommt dem Langzeit-EEG mit Videoüberwachung des Patienten zu, da nur durch die Korrelation des klinischen Bildes mit dem EEG-Befund eine genaue Lokalisation der Epileptogenese möglich ist.

Ziel der Epilepsietherapie ist, die Anfallsfreiheit des Patienten zu erreichen. Mittel der ersten Wahl sind dabei antikonvulsive Medikamente, die die Anfallschwelle modifizieren und damit Anfälle verhindern. Sie stellen somit keine kausale Therapie dar. Die Patienten werden unter Berücksichtigung von Wirksamkeit, Nebenwirkungen und Verträglichkeit zunächst auf eine Monotherapie mit einem Antikonvulsivum eingestellt. Darunter kommt es, unabhängig von dem eingesetzten Antikonvulsivum, bereits bei ca. 50 \% der Patienten zur Anfallsfreiheit. Bei Versagen der Therapie erfolgt in der Regel eine Umstellung des Patienten auf eine Monotherapie mit einem anderen Antikonvulsivum, die bei weiteren $20 \%$ der Patienten zu einer Anfallsfreiheit führt (Kwan und Brodie 2000).

Prinzipiell ist bei Versagen der medikamentösen Therapie einer fokalen Epilepsie (Pharmakoresistenz) auch die Möglichkeit eines epilepsiechirurgischen Eingriffs zu nennen, der die Entfernung des Epilepsiefokus ohne die Verursachung zusätzlicher neurologischer Defekte zum Ziel hat und dabei einen kausalen Ansatz verfolgt.

In den Empfehlungen der neuesten Leitlinie der Deutschen Gesellschaft für Neurologie (DGN) wird der Stellenwert der Epilepsiechirurgie gestärkt. Laut DGN-Leitlinie erfolgt die Vorstellung von Epilepsiepatienten zu einem epilepsiechirurgischen Eingriff bisher sehr spät. Der Begriff der Pharmakoresistenz wurde in Anlehnung an die Empfehlung der ILEA neu definiert. Demzufolge besteht eine Pharmokoresistenz, wenn die Therapie mit zwei verschiedenen geeigneten Antikonvulsiva erfolglos war (Kwan et al. 2010). Die neue DGN-Leitlinie fordert die Vorstellung von Patienten mit einer Pharmakoresistenz in einem Zentrum zur Evaluation der Operabilität (Diner und Weimar 2012).

Voraussetzung für einen epilepsiechirurgischen Eingriff ist eine umfassende prächirurgische Diagnostik. Besondere Bedeutung liegt dabei auf der genauen Fokuslokalisation und der Fragestellung, ob der epileptogene Fokus, ohne zusätzliche 
neurologische Schäden zu verursachen, entfernt werden kann. Neben speziellen MRTUntersuchungen und neuropsychologischen Testverfahren ist bei bestimmten Patienten auch die vorübergehende pharmakologische Inaktivierung bestimmter Hirnareale durch den Wada-Tests indiziert (Stefan 2000).

\subsubsection{DER WADA-TEST}

Einige kognitive Hirnleistungen wie Sprache und Gedächtnis sind auf die beiden Hemisphären verteilt, wobei meist eine Hemisphäre dominant bezüglich dieser Funktion ist. Eine genaue präoperative Bestimmung der Sprachlateralisierung ist für die Einschätzung des Verletzungsrisikos wichtiger Strukturen während eines epilepsiechirurgischen Eingriffs zur Minimierung der Gefahr postoperativer Sprachdefizite von großer Bedeutung.

Der Wada-Test ist der Goldstandard zur Untersuchung der Lateralität kognitiver Hirnleistungen. Bei dem wachen Patienten wird zunächst eine Angiographie durchgeführt und anschließend ein Barbiturat über eine der beiden Aa. carotis internae in die A. cerebri media einer Hemisphäre injiziert. Die betreffende Hemisphäre wird narkotisiert, so dass die kognitive Funktion der Hemisphäre indirekt durch den Ausfall der Hirnleistung in der narkotisierten Hemisphäre bzw. im Vergleich mit der kontralateralen Hemisphäre identifiziert werden kann (Wada und Rasmussen 1960).

Eine Weiterentwicklung des oben beschriebenen konventionellen Wada-Tests stellt der selektive Wada-Test dar. Durch die Injektion des Barbiturates in eine kleinere Hirnarterie, wie die A. cerebri posterior, die A. choroidea anterior oder kleinere Äste der A. cerebri media, können einzelne Regionen einer Hemisphäre narkotisiert werden. Neben der Bestimmung der Lateralität kognitiver Funktionen ist dadurch auch die Untersuchung der Funktion einzelner Hirnregionen möglich (Urbach et al. 2002).

Nachteile des Wada-Tests sind jedoch die Invasivität dieses Tests mit der Gefahr von assoziierten Komplikationen wie Blutungen oder Thrombosen mit daraus resultierenden neurologischen Ausfällen. In der Regel ist dadurch die Wiederholung des Tests nicht möglich. Darüber hinaus können Informationen, die über die relative Verteilung der Sprachfunktion hinausgehen, mit dem Wada-Test nicht gewonnen werden. Diese sind jedoch für eventuelle neurochirurgische Eingriffe von großer Bedeutung.

Als Alternative zum Wada-Test ermöglichen bildgebende Verfahren die nichtinvasive Untersuchung der Lateralität kognitiver Hirnfunktionen. Neue Techniken der Magnetresonanztomografie wie fMRT und DTI (siehe Kapitel 1.4.2 Seite 13 und 1.4.4 Seite 15) können ohne Risiko mehrfach wiederholt werden. Sie bieten außerdem in Kombination mit anatomischen Daten die Möglichkeit der genauen Lokalisation sprachrelevanter Kortexareale und könnten somit zukünftig auch für die Planung von Resektionsgrenzen in der Epilepsiechirurgie von großem Nutzen sein. 


\subsection{MRT}

Die Magnetresonanztomografie (MRT), auch Kernspintomografie genannt, erlaubt eine Schnittbildrekonstruktion des menschlichen Körpers ohne die Verwendung von Röntgenstrahlung.

\subsubsection{FUNKTIONSWEISE DER MRT}

Atome mit unpaarigen Protonen und Neutronen besitzen einen Eigendrehimpuls (spin), aus dem ein magnetisches Dipolmoment resultiert. Dieses Phänomen des Kernspins ist die Grundlage für die MRT. Die MRT arbeitet vor allem mit dem spin des Kerns des Wasserstoffatoms $(\mathrm{H}+)$, der aus einem einzelnen Proton besteht. Wasserstoff ist Bestandteil von Fett und Wasser und macht daher einen großen Anteil des menschlichen Organismus aus.

Wird der Körper in das starke äußere Magnetfeld eines Tomografen bewegt, richten sich die Protonen und damit auch die Dipolmomente parallel und antiparallel zum äußeren Magnetfeld aus. Der Anteil der parallel ausgerichteten Protonen überwiegt dabei leicht aufgrund der energetisch günstigeren Ausrichtung (Boltzmann-Verteilung). Dadurch entsteht die Längsmagnetisierung, eine parallel zum äußeren Magnetfeld ausgerichtete Gesamtmagnetisierung. Unpaarige Atome in einem äußeren Magnetfeld führen außerdem eine Kreiselbewegung um die Hauptfeldlinie dieses äußeren Magnetfeldes aus (Präzession). Die Kreiselfrequenz wird auch als Präzessionsfrequenz oder Lamorfrequenz bezeichnet und ist vom Magnetfeld und den Teilchen abhängig.

Während einer MR-Messung wird den Protonen Energie in Form eines Hochfrequenzimpulses zugeführt. Bei Übereinstimmen der Frequenz des elektromagnetischen Impulses mit der Lamorfrequenz der Wasserstoffprotonen herrschen Resonanzbedingungen vor, unter denen eine Energieübertragung auf Protonen möglich wird. Die angeregten Protonen ändern ihre Richtung, so dass die ursprüngliche Längsmagnetisierung ausgelenkt wird und eine Quermagnetisierung entsteht. Zudem wird die Präzession der Protonen synchronisiert. Sobald der Impuls abgeschaltet wird, relaxieren die angeregten Protonen und kehren, unter Freisetzung der zuvor absorbierten Energie, wieder in ihren Urzustand zurück (Relaxation). Die freigesetzte Energie kann als Relaxationssignal von einer Empfangsspule detektiert und zur Bildgebung verwendet werden.

Um ein ausreichend großes Messsignal zu erhalten, müssen mehrere Impulse in Folge an den Körper abgegeben werden. Die Zeit zwischen zwei Impulsen ist die Repetitionszeit (TR: time of repetition), die Zeit zwischen Impuls und Messung des Signals ist die Echozeit (TE: time of echo). 


\subsubsection{RELAXATION}

Die Relaxation wird durch zwei Zeitkonstanten beschrieben: die Längsrelaxation (T1) und die Querrelaxation (T2).

Der Kontrast einer MR-Bildgebung wird durch die T1-Relaxation und die T2-Relaxation sowie die Protonendichte bestimmt. Durch Variation der Echozeit und der Repetitionszeit kann die Betonung eines dieser Kontrastparameter (Wichtung) vorgenommen werden, so dass kontrastreiche Bilder entstehen, die zu diagnostischen Zwecken genutzt werden können.

\subsubsection{ORTSKODIERUNG}

Die Positionsbestimmung der Signale der relaxierenden Kerne geschieht mit Hilfe der Ortskodierung. Dazu werden Gradientenspulen geschaltet, die das statische Magnetfeld des Tomografen mit Magnetfeldern geringerer Feldstärke überlagern. Die Gradienten können in allen drei Raumebenen geschaltet werden und verändern auf diese Weise gezielt die Stärke des statischen Magnetfeldes und damit auch die Larmorfrequenz einer umschriebenen Schicht. Ein Hochfrequenzimpuls mit der übereinstimmenden Frequenz regt auf diese Weise nur die Protonen dieser Schicht an. Die Position der angeregten Protonen kann mit Kenntnis der geschalteten Gradienten bestimmt werden.

\subsubsection{EPI-SEQUENZ}

Die Abfolge von Hochfrequenzimpulsen und geschalteten Gradienten zur Ortskodierung wird als Sequenz bezeichnet. Die echoplanare Bildgebung (EPI: echoplanar imaging) bedient sich einer speziellen Sequenz, die zu einer Verkürzung der Bildaufnahmezeiten in den Millisekundenbereich führt. Mit der EPI-Sequenz ist eine hohe zeitliche Auflösung der MRT möglich, die es erlaubt, Kortexaktivierung darzustellen. Daher wurde diese Sequenz in der vorliegenden Arbeit verwendet.

\subsubsection{FMRT}

Die fMRT ist ein nicht-invasives Verfahren zur Darstellung individueller neuronaler Aktivität der grauen Substanz. Das Prinzip der fMRT beruht auf der Darstellung der Signalunterschiede zwischen aktivierten und nicht aktivierten Kortexarealen. Die Aktivitätsunterschiede können in der fMRT durch Kontrastmittel, die dem Patienten exogen zugeführt werden, oder mit Hilfe des zerebralen Blutflusses, der als endogenes Kontrastmittel dient, abgebildet werden. Die Methode, die sich des zerebralen Blutflusses bedient, wird als BOLD (blood oxygen level dependent) fMRT bezeichnet und ist eine etablierte und häufig angewendete Methode in der funktionellen Hirnforschung. 


\subsubsection{BOLD FMRT}

Die Grundlage der BOLD fMRT bilden die Kopplung der neuronalen Aktivität an die Hämodynamik sowie die gut untersuchte Annahme, dass das fMRT-Signal proportional zum Maß der lokalen neuronalen Aktivität ist (Logothetis et al. 2001). Auf diese Weise kann Hirnaktivität ohne die Verwendung exogener Kontrastmittel dargestellt werden.

Mit Sauerstoff beladenes Hämoglobin (Oxyhämoglobin, Oxy-Hb) und Hämoglobin, das den Sauerstoff wieder abgegeben hat (Desoxyhämoglobin, Desoxy-Hb), besitzen unterschiedliche magnetische Eigenschaften, die durch Wechselwirkungen des Eisenatoms des Hämoglobins mit einem Sauerstoffmolekül bestimmt werden (Ogawa et al. 1990). Oxy-Hb besitzt schwach diamagnetische Eigenschaften, die zu einer Verstärkung des MR-Signals führen, während Desoxy-Hb paramagnetische Eigenschaften besitzt, die im angrenzenden Gewebe eine Inhomogenität des magnetischen Feldes erzeugen und zu einer Verminderung des MR-Signals führen.

Neuronale Aktivität bewirkt einen gesteigerten Sauerstoffverbrauch, der durch einen erhöhten regionalen Blutfluss überkompensiert wird. Aufgrund der Trägheit der Hämodynamik erfolgt die Überkompensation mit einer Verzögerung von wenigen Sekunden. Durch den erhöhten regionalen Blutfluss und das erhöhte Blutvolumen steigt auch die Menge des $\mathrm{Oxy}-\mathrm{Hbs}$ in der aktivierten Hirnregion. Diese drei Komponenten können mit dem BOLD-Verfahren als Verstärkung des MR-Signals registriert werden. Die BOLD fMRT stellt also nicht direkt die Aktivierung von Neuronen dar, sondern hämodynamische Veränderungen im Gehirn aufgrund eines Stimulus.

\subsubsection{DIE hÄMODYNAMISCHE ANTWORTFUNKTION}

Das BOLD-Signal repräsentiert die hämodynamische Antwort auf einen Stimulus und folgt dabei einer typischen Funktion, der hämodynamischen Antwortfunktion (HRF: haemodynamic response function).

Der Kontrast zwischen aktiviertem Hirngewebe und umgebendem Gewebe im BOLD fMRT ist so gering, dass er lediglich im Vergleich zu Ruhephasen derselben Hirnregion sichtbar wird. Daher werden für BOLD-Untersuchungen häufig Testparadigmen im Blockdesign verwendet, in denen sich Aktivitätsphasen mit Ruhephasen abwechseln. Die Signaldifferenz der unterschiedlichen Aktivierungsphasen kann dann zur Generierung von funktionellen Aktivierungskarten genutzt werden.

\subsubsection{SIGNAL-ZU-RAUSCH-VERHÄLTNIS}

Das Signal einer MR-Messung wird zu einem bestimmten Grad vom Hintergrundrauschen, das durch zufällige Signalschwankungen entsteht, verunreinigt.

Ein Maß für die Bildqualität von MR-Messungen ist daher das Signal-zu-RauschVerhältnis (SNR: signal to noise ratio). Das SNR wird vor allem durch die Auswahl der MR-Sequenz beeinflusst, da die Sequenzen sich in ihren Repetitionszeiten und Auflösungen voneinander unterscheiden. Die Auflösung einer fMRT wird z. B. niedriger 
gewählt als die Auflösung von anatomischen Aufnahmen, da das SNR sonst nicht ausreichen würde, um den BOLD-Kontrast abzubilden. Eine deutliche Erhöhung des SNR lässt sich über Messwiederholungen in einem Blockdesign erreichen.

\subsubsection{AUSWERTUNG VON MRT-DATEN}

Die Aktivierung kann auf Basis einzelner Voxel oder auf Grundlage ganzer Voxelverbände, den sogenannten Regionen von Interesse (ROI: region of interest), ausgewertet werden. Eine ROI ist eine eng umschriebene Menge an Voxeln einer Aktivierungskarte, die untersucht und zur statistischen Auswertung herangezogen werden.

\subsubsection{DAS TALAIRACHKOORDINATENSYSTEM}

Gehirne einzelner Individuen unterscheiden sich voneinander in ihrer Länge, Breite und Höhe. Durch eine räumliche Normalisierung können Ergebnisse bildgebender Studien trotz dieser interindividuellen anatomischen Unterschiede vergleichbar gemacht werden.

Jean Talairach und Pierre Tournoux erarbeiteten das Talairachkoordinatensystem, ein räumlich standardisiertes Koordinatensystem, an das die anatomischen Strukturen jedes menschlichen Gehirns proportional angepasst werden können. Der Talairachatlas erschien erstmals 1988 in englischer Sprache und dient seitdem als weltweit anerkanntes Standardkoordinatensystem, mit dessen Hilfe funktionelle bildgebende Studien über das menschliche Gehirn durchgeführt werden (Talairach und Tournoux 1988). Erst die Anpassung an ein Standardkoordinatensystem ermöglicht, interindividuelle Unterschiede in der Neuroanatomie auszugleichen und Gruppenanalysen über die Funktion von Gehirnarealen durchzuführen.

\subsubsection{DiffusionsgeWICHTETE MRT}

Mit Hilfe der diffusionsgewichteten MRT können Informationen über die Mikrostruktur der weißen Substanz des Gehirns gewonnen werden. Die Diffusionseigenschaften in der weißen Substanz können in Karten visualisiert werden und erlauben Rückschlüsse auf die Faserverläufe zwischen verschiedenen Kortexregionen, wie z.B. dem Brocaund dem Wernicke-Sprachzentrum.

\subsubsection{DWI}

Diffusionsgewichtete MRT (DWI: diffusion weighted imaging) ist ein MR-Verfahren, das in vivo Bilder organischer Gewebe auf Grundlage der Diffusionseigenschaften von Wassermolekülen erstellt (Le Bihan et al. 1986).

Die Diffusion von Wassermolekülen wird durch die Brown'sche Molekularbewegung beschrieben. Ihr zufolge bewegen sich Wassermoleküle ohne äußere Grenzen frei in 
alle Richtungen. Herrschen Bedingungen vor, unter denen eine freie Diffusion in alle Richtungen möglich ist, so wird dieser Zustand Isotropie genannt.

In der weißen Substanz bilden die Zellmembranen der Nervenfasern jedoch physische Grenzen, die zu einer Diffusionseinschränkung der Wassermoleküle führen. Die Diffusionseinschränkung durch die Membranen der Axone ist dabei senkrecht zum Axonverlauf größer als parallel zu ihrem Verlauf. Die eingeschränkte und damit gerichtete Diffusion wird als Anisotropie bezeichnet (Moseley et al. 1991). Aus diesen Diffusionsunterschieden lassen sich Hinweise auf die Struktur der weißen Substanz ableiten.

\subsubsection{DTI}

Die Diffusions-Tensor-Bildgebung (DTI: diffusion tensor imaging) ist eine komplexere Variante der DWI. Sie erfasst neben der Stärke der Diffusion von Wassermolekülen, die in dem Diffusionskoeffizienten (ADC = apparent diffusion coefficient) ausgedrückt werden kann, auch die Diffusionsrichtung und erlaubt Untersuchungen der Mikrostruktur der weißen Substanz in einer Vielzahl von Raumrichtungen. Über einen Tensor wird das dreidimensionale Diffusionsverhalten in einem Voxel charakterisiert und die Richtung der größten Diffusion angegeben. Die Fraktionelle Anisotropie (FA) ist ein Index, der die Eigenschaften dieses Tensors abstrahiert und damit ermöglicht, Informationen über das Ausmaß der Anisotropie zu berechnen (Mori und van Zijl 2002). Die FA umfasst dabei einen Wertebereich von 0 bis 1 , wobei 0 maximale Isotropie und 1 maximale Anisotropie beschreibt. Regionen, in denen aufgrund vieler paralleler Diffusionsbarrieren eine hohe gerichtete Diffusion stattfindet, wie z.B. in Faserbündeln der weißen Substanz, zeigen hohe Anisotropiewerte. Hirnstrukturen, die hingegen aus vielen Zellkörpern bestehen, wie die graue Substanz, zeigen aufgrund der wenigen parallelen Diffusionsbarrieren niedrige Anisotropiewerte (Abbildung 3).
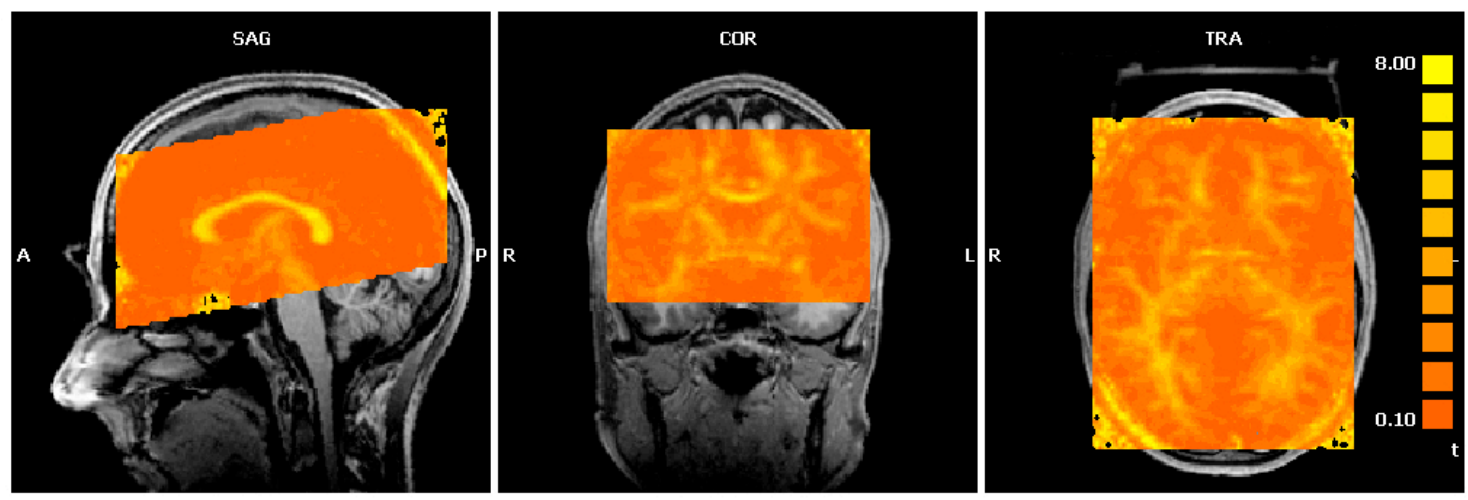

Abbildung 3: FA-Karte. Regionen mit höherer Anisotropie (z.B. der Balken) werden mehr gelb dargestellt, Regionen mit geringerer Anisotropie (z.B. die graue Substanz) werden mehr orange dargestellt. Achtung: für eine bessere Darstellung wurde die FA-Skala in dieser Abbildung um den Faktor 10 vergrößert. Eine $\mathrm{FA}=8$ auf der Karte entspricht einer wirklichen $\mathrm{FA}=0,8$. 
In farbkodierten FA-Karten kann auch die Richtung der Diffusion wiedergegeben werden (Abbildung 4).
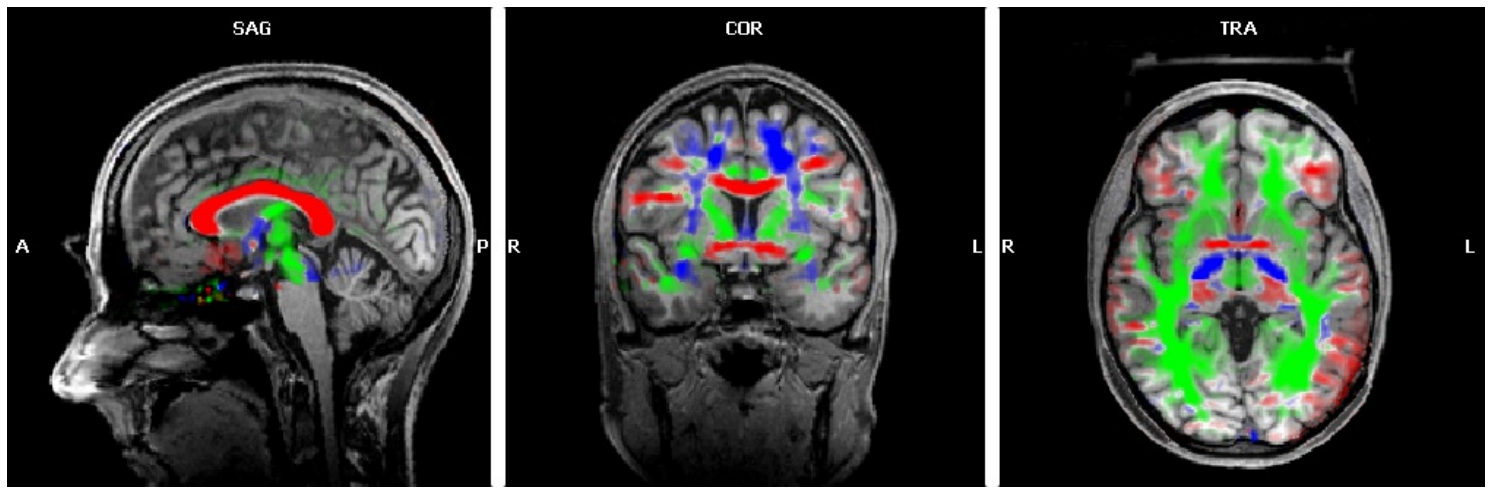

Abbildung 4: Farbkodierte FA-Karte. Blau zeigt Diffusion in Richtung der superior-inferioren Achse an, grün in Richtung der anterior-posterioren Achse und rot in Richtung der Links-Rechts-Achse.

\subsection{Ziel der vorliegenden Arbeit}

Ziel der vorliegenden Arbeit war die exakte Bestimmung der sprachdominanten Hemisphäre bei Patienten mit fokaler Epilepsie mit Hilfe von nichtinvasiven bildgebenden Verfahren.

Bislang gilt der Wada-Test als Goldstandard in der Lateralitätsbestimmung. Die fMRT ist eine nichtinvasive Alternative in der Lateralitätsbestimmung, die darüber hinaus auch die Darstellung der funktionellen Sprachareale ermöglicht. Studien haben eine 90\%ige Übereinstimmung der Ergebnisse des Wada-Tests mit denen der fMRT hinsichtlich der Bestimmung der sprachdominanten Hemisphäre gezeigt (Adcock et al. 2003; Binder et al. 1996; Woermann et al. 2003). Diese Übereinstimmung ist jedoch in der präoperativen Diagnostik der Epilepsiechirurgie nicht ausreichend, so dass fMRTVerfahren in dieser Form den Wada-Test noch nicht ersetzen können.

In der vorliegenden Arbeit wurde ein fMRT-Verfahren mit einem DTI-Verfahren kombiniert. Mit dieser Methode sollte gezeigt werden, ob in der weißen Substanz eine strukturelle Asymmetrie besteht, welche die Sprachlateralisierung der grauen Substanz widerspiegelt und eine geeignete Ergänzung in der Diagnostik darstellt.

Außerdem wurde erforscht, wie sich die Lokalisation der sprachrelevanten Kortexareale bei Patienten mit fokaler Epilepsie von der Lokalisation bei gesunden Kontrollprobanden unterscheidet und ob sich bei Patienten mit fokaler Epilepsie ein anatomisches Korrelat der weißen Substanz für die in der fMRT veränderte funktionelle Kortexaufteilung findet. 


\section{Material und Methode}

Die vorliegende Methode ist im Rahmen einer Studiengruppe erarbeitet worden, welche die Sprachlateralisierung mittels funktioneller Magnetresonanztomografie und Diffusionstensorbildgebung erforschte. Die vorliegende Dissertation untersuchte die Sprachlateralisierung bei Epilepsiepatienten. Die Daten des gesunden Kontrollkollektivs (Gruppe N) wurden dissertationsübergreifend in der Studiengruppe erhoben (siehe Tabelle 40) und in einer weiteren Dissertation, welche die Sprachlateralisierung bei Patienten mit idiopathischem Stottern untersuchte, verwendet (Bonnkirch (in Vorbereitung)). Die Erhebung und Bearbeitung der Daten des Patientenkollektivs mit fokaler Epilepsie (Gruppe E) sowie die statistische Auswertung und Gegenüberstellung beider Kollektive erfolgten in dieser Dissertation unabhängig und eigenständig von der Kooperation in der Studiengruppe.

\subsection{Probanden}

Voraussetzung zur Aufnahme der Probanden in das Patientenkollektiv (Gruppe E) war die gesicherte Diagnose einer Temporallappenepilepsie. Zur Klassifikation der Epilepsieform wurde bei allen Patienten mit fokaler Epilepsie vor Beginn der Studie eine Epilepsiediagnostik nach dem Standard der Abteilung für Klinische Neurophysiologie der Universitätsmedizin Göttingen (UMG) durchgeführt. Diese beinhaltete eine fachärztliche Untersuchung in der Epilepsieambulanz der Klinischen Neurophysiologie der UMG und die sichere Lokalisation des Epilepsieherdes durch ein Routine-EEG, ein Video-EEG und ein kraniales MRT.

Das Patientenkollektiv bestand aus 12 Probanden (3 Frauen und 9 Männer) mit sicher diagnostizierter fokaler Epilepsie im Alter zwischen 21 und 57 Jahren und einem Durchschnittsalter von 39,6 Jahren (Tabelle 1). Alle Teilnehmer des Patientenkollektivs sprachen Deutsch als Muttersprache.

Neun der untersuchten Patienten litten an einer linksseitigen Temporallappenepilepsie (Tabelle 2). Als Ursache für die fokale Epilepsie konnte bei fünf der Patienten eine linksseitige Hippokampussklerose im MRT nachgewiesen werden und bei einem weiteren Patienten zeigte sich im MRT eine Seitenasymmetrie der Hippokampusformation zuungunsten der linken Hemisphäre. Des Weiteren konnte bei einem Patienten linkstemporal ein Abszess bei bekannter Meningoenzephalitis in der Vorgeschichte im MRT nachgewiesen werden. Die Ätiologie der fokalen Epilepsie der übrigen beiden Patienten mit linkshemisphärischer Epileptogenese war bisher unbekannt. 
Die übrigen drei Patienten wiesen keine linkshemisphärische Epileptogenese auf. Bei zwei von innen bestand am ehesten eine rechtshemisphärische Epileptogenese. Lediglich einer Patientin konnte bezüglich der Epileptogenese keine Hemisphäre zugeordnet werden. Die Ätiologie der fokalen Epilepsien von zwei Patienten war bislang unklar. Bei einem Patienten war im MRT eine postentzündliche Läsion rechts temporal nach bekannter Varizella-Zoster-Enzephalitis in der Vergangenheit aufgefallen.

Fünf Patienten waren medikamentös auf eine Monotherapie, drei Patienten auf eine Zweifach-Kombination und vier Patienten auf eine Kombination aus drei verschiedenen Antikonvulsiva eingestellt. Insgesamt wurden vier Patienten des untersuchten Kollektivs für einen epilepsiechirurgischen Eingriff in Betracht gezogen.

Die Anfallsfrequenz der untersuchten Patienten schwankte zwischen ein Mal pro Jahr und drei bis vier Mal pro Woche.

Das Kontrollkollektiv (Gruppe N) bestand aus 23 Probanden (6 Frauen und 17 Männer) im Alter zwischen 22 und 59 Jahren und einem Durchschnittsalter von 33,8 Jahren, bei denen anamnestisch keine neurologischen Erkrankungen vorlagen (Tabelle 1). Ebenso sprachen alle Teilnehmer des Kontrollkollektivs Deutsch als Muttersprache.

Die Studiendurchführung wurde von der Ethikkommission der UMG geprüft und bewilligt (laufende Antragsnummer 01/04/06). Alle Probanden wurden vor Beginn der MRT-Messung über die Ziele der Studie, den Ablauf der Messung und die Risiken einer MRT-Untersuchung, sowohl mündlich als auch schriftlich, aufgeklärt und die Einwilligung wurde schriftlich dokumentiert.

\subsection{Händigkeit}

Zur Ermittlung der Händigkeit der Probanden wurde vor Beginn der MRTUntersuchung eine deutsche Übersetzung des Edinburgh-Händigkeitsinventars (EHI) (Oldfield 1971) bei jedem Probanden durchgeführt.

Die Händigkeit wurde durch einen Fragebogen über 10 alltägliche Tätigkeiten berechnet, deren Durchführung neutral für Geschlecht, Nationalität und sozioökonomischen sowie kulturellen Hintergrund war. Für jede Tätigkeit kreuzten die Probanden an, ob sie diese Tätigkeit nur mit rechts (++ für rechts), nur mit links (++ für links), eher mit rechts (+ für rechts), eher mit links (+ für links) oder mit beiden Händen gleich häufig (+ für rechts und + für links) durchführten. Aus diesen Angaben wurde dann der Lateralitätsquotient nach folgender Formel gebildet:

$H=100 \times(R-L) /(R+L)$ 
$R$ gibt dabei die Anzahl der Kreuze für rechts und $L$ die Anzahl der Kreuze für links an. Die Händigkeit $(H)$ wurde auf einer quantitativen Skala mit Werten von -100 bis +100 angegeben, wobei -100 einem absoluten Linkshänder und +100 einem absoluten

Rechtshänder entspricht. Schließlich wurden die Probanden in die Gruppen Rechtshänder $(H \geq+50)$ und Nicht-Rechtshänder $(H<+50)$ eingeteilt (Szaflarski et al. 2002).

Die Händigkeit wurde mit Hilfe folgender Internetadresse berechnet:

http://www.cse.yorku.ca/course_archive/2006-07/W/4441/Edinburghlnventory.html

\subsection{Neuropsychologisches Testparadigma}

Zur Darstellung der funktionellen Sprachlateralisierung wurde ein etablierter Sprachtest im Blockdesign verwendet (Stippich et al. 2003). Dieser Test setzte sich aus zwei verschieden Stimulationsparadigmen zusammen, bei denen der Stimulus während der MRT-Messung den Probanden visuell über eine LCD-Brille präsentiert wurde. Jedes Paradigma bestand aus Phasen, in denen sich aktive Stimuli und passiver Kontrollstimulus abwechselten, sowie einer vorangehenden passiven Kontrollphase zu Beginn eines jeden Paradigmas. Jede dieser Phasen dauerte 36 Sekunden, so dass pro Paradigma eine Gesamtmessdauer von 5:40 Minuten bestand.

Das erste Paradigma "Satz" testete die Satzgenerierung. Den Probanden wurden in der aktiven Phase Stimuli in Form von pseudorandomisierten Bildern mit einfachen Gegenständen über die LCD-Brille eingespielt. Alle drei Sekunden erschien ein neues Bild und die Probanden sollten zu dem jeweils aktuellen Bild Sätze "laut" im Kopf denken, diese aber nicht laut aussprechen. Der aktiven Phase folgte eine passive Kontrollphase mit einem unveränderten Ruhebild, das aus einem grauen Bildschirm mit Fixationshilfe in Form eines Punktes bestand. In dieser Phase sollten die Probanden entspannen und möglichst keine Worte oder Sätze denken.

Das zweite Paradigma „Wort" testete die Wortgenerierung. Den Probanden wurden in der aktiven Phase geschriebene Substantive über die LCD-Brille gezeigt, zu denen möglichst viele assoziierte Substantive „laut“ im Kopf gedacht, aber wiederum nicht laut ausgesprochen werden sollten. Auch in diesem Paradigma erschien alle drei Sekunden ein neues Substantiv. Der aktiven Phase folgte eine passive Kontrollphase mit einem unveränderten Ruhebild (Abbildung 5). 

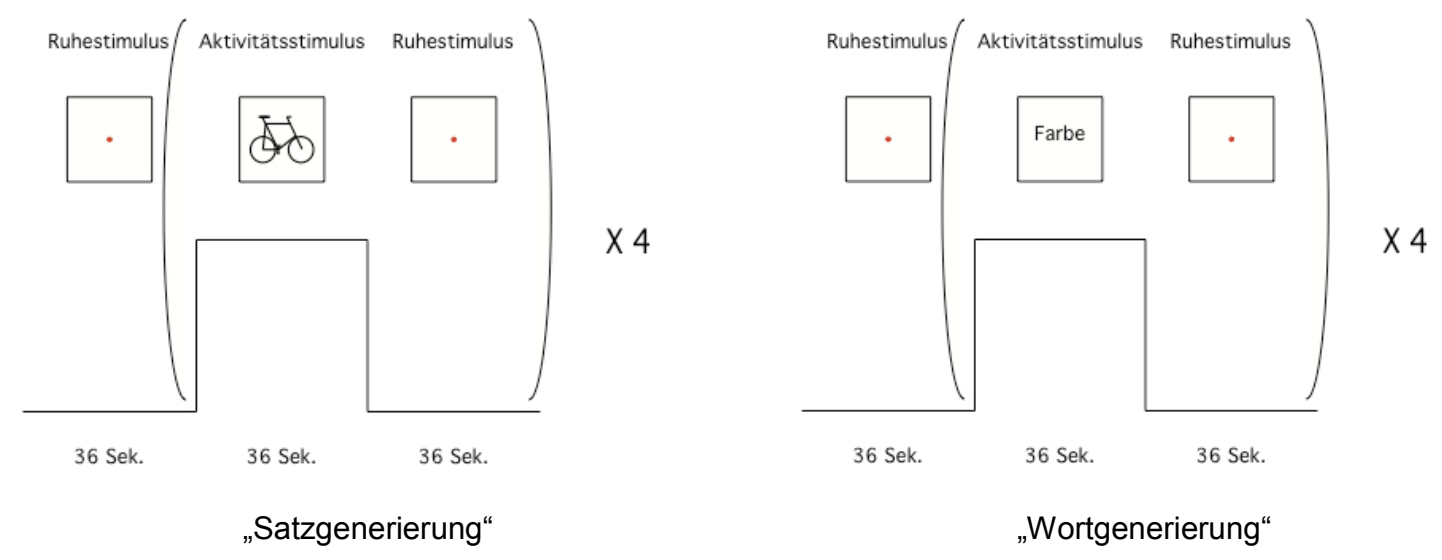

Abbildung 5: Schematische Darstellung der Stimulationsparadigmen „Satzgenerierung“ und „Wortgenerierung“.

Stippich et al. (2003) bewiesen, dass beide Testparadigmen zu einer fast identischen Aktivierung der grauen Substanz in der fMRT führten und dass aufgrund der unterschiedlichen Schwierigkeitsstufe der Testparadigmen („Wortgenerierung“ und „Satzgenerierung“) die Ergebnisse bei einer Kombination der beiden Paradigmen unbeeinflusst von den kognitiven Fähigkeiten der Versuchspersonen waren.

Damit die Beeinflussung des MRT-Experiments durch Bewegungsartefakte möglichst gering blieb, wurden die Probanden angewiesen, die Aufgaben still zu bearbeiten und dabei den Kopf und die Zunge nicht zu bewegen.

\subsection{MRT-Datenerfassung}

Alle MRT-Aufnahmen wurden mit einem 3-Tesla-Magnetresonanztomografen mit 8Kanal-Kopfspule (Siemens Magnetom Trio, Erlangen, Germany) erstellt. Zum Lärmschutz trugen die Probanden während der gesamten MRT-Messung Kopfhörer. Die Vitalparameter wurden mittels Pulsoxymetrie überwacht. Die MRT-Messung bestand aus verschiedenen Sequenzen, die unterschiedliche Informationen lieferten.

Durch Variation der Wichtungsparameter kann eine Sequenz in T1-Wichtung genaue anatomische Verhältnisse darstellen oder in T2*-Wichtung funktionelle Informationen des Kortex in Aktivierungskarten abbilden, die den Zeitverlauf des BOLD-Signals in dreidimensionalen Bildpunkten, den Voxeln, zeigen.

Während jedes Messblocks wurde das gesamte Volumen abgebildet, welches der gesamten untersuchten Körperregion, in diesem Fall dem Gehirn, entsprach. Jedes Volumen wurde wiederum in mehreren Schichten abgebildet, die aus einzelnen Voxeln, der kleinste Einheit, die mit einer fMRT dargestellt werden kann, bestand. Ein Voxel ist ein dreidimensionales Volumenelement, das dem Produkt aus einem zweidimensionalen Bildpunkt (Pixel) und der Schichtdicke entspricht $($ Voxel $=$ Pixel $\mathrm{x}$ Schichtdicke). 


\subsubsection{ANATOMISCHE REFERENZBILDER}

Zunächst wurden je eine T1-gewichtete und eine T2-gewichtete Aufnahme des gesamten Kopfes mit isotroper Auflösung von $1 \mathrm{~mm}^{3}$ aufgezeichnet (3D Turbo Fast Low Angle Shot (FLASH), TR: $1950 \mathrm{~ms}$, Inversionszeit: $1100 \mathrm{~ms}$, TE: $3.93 \mathrm{~ms}$, Flipwinkel: $12^{\circ}$ ).

Die T1-gewichteten Aufnahmen stellten die anatomische Referenz für Informationen über die Hirnaktivität dar, welche aus den vorverarbeiteten $T 2^{*}$-gewichteten Daten hervorgingen. In der Auswertung konnten daher funktionelle Aktivierungen genauen anatomischen Strukturen zugeordnet werden.

\subsubsection{FUNKTIONELLE MRT-BILDER}

Die funktionellen Daten wurden in T2*-Wichtung mit Gradienten-Echo EPI-Technik und einer Schichtauflösung von $2 \times 2 \mathrm{~mm}$ und $4 \mathrm{~mm}$ Schichtdicke erstellt (TR: $2000 \mathrm{~ms}$, TE: $36 \mathrm{~ms}$, Flipwinkel: $70^{\circ}$, Erfassungsmatrix: $84 \times 128$ ).

\subsubsection{DIFFUSIONSGEWICHTETE MRT-BILDER}

Die Diffusionstensor-Bilddaten wurden mit einer diffusionsgewichteten partiellen Fourier single-shot stimulated echo acquisition mode (STEAM) MagnetresonanzSequenz und einer Ebenenauflösung von 2,2 x 2,2 $\mathrm{mm}^{2}$ und 2,2 mm Schichtdicke erstellt (TR: 16,2 s, TE: $50 \mathrm{~ms}$, ausgelesener Flipwinkel: $10^{\circ}, 38$ axiale Schichten, 3 Mittelungen) erstellt (Rieseberg et al. 2005).

Das Protokoll verglich eine Referenzaufnahme ohne Diffusionswichtung mit diffusionsgewichteten Aufnahmen mit einem b-Wert von $1000 \mathrm{~s} / \mathrm{mm}^{2}$ entlang 24 Richtungen alternierender Polarität (Ikosaeder-Schema).

\section{5 fMRT-Datenverarbeitung}

Die funktionellen Daten wurden zunächst vorverarbeitet (Vorverarbeitung = preprocessing). Um einen räumlichen Vergleich der Bilddatensätze in einem gemeinsamen Referenzraum zu ermöglichen, wurden die Daten unterschiedlicher Aufnahmemodalität mit Hilfe einer Koregistrierung in ein gemeinsames Koordinatensystem eingebettet.

Die fMRT-Daten wurden mit Brain Voyager QX 1.10.2 (Brain Innovation, Maastricht, The Netherlands) aufbereitet und visualisiert (Goebel et al. 2006). 
Rohdaten:

- $\quad 1 \times$ T1-gewichtete anatomische Aufnahmen, isotrope Auflösung $1 \mathrm{~mm}^{3}, 176$ Schichten, in FLASH-Technik

- $\quad 1 \times$ T2-gewichtete anatomische Aufnahmen, isotrope Auflösung $1 \mathrm{~mm}^{3}, 144$ Schichten in FLASH-Technik

- $1 \times$ T2*$^{*}$-gewichtete funktionelle Aufnahmen, Schichtauflösung $2 \times 2 \mathrm{~mm}$, Schichtdicke $4 \mathrm{~mm}, 162$ Volumen zu je 20 Schichten (Erfassungsmatrix: 84 x 128), in EPI-Technik während des Sprachtests „Satzgenerierung“

- $1 \times$ T2*$^{*}$-gewichtete funktionelle Aufnahmen, Schichtauflösung $2 \times 2 \mathrm{~mm}$, Schichtdicke $4 \mathrm{~mm}, 162$ Volumen zu je 20 Schichten (Erfassungsmatrix: 84 x 128), in EPI-Technik während des Sprachtests „Wortgenerierung“

Zunächst wurde aus den T1-Daten, die als einzelne zweidimensionale Bilddateien vom Scanner aufgenommen und ausgegeben wurden, ein anatomisches 3D-Projekt erstellt.

Die T1-gewichteten Daten, die später als anatomische Referenz für die funktionellen Daten dienten, wurden anschließend auf das Talairachsystem normalisiert. Den Ursprung des Koordinatensystems (0/0/0) stellte die vordere Kommissur (Commissura anterior $=\mathrm{AC}$ ) in der Medianebene dar. Die drei orthogonal zueinander stehenden Raumrichtungen (Transversalachse $=x$-Achse, Sagittalachse $=y$-Achse, Longitudinalachse $=z$-Achse) kreuzten sich in diesem Punkt. Die Verbindungslinie der hinteren Kommissur (Commissura posterior $=\mathrm{PC}$ ) mit der vorderen Kommissur bildete die Y-Achse des Talairachkoordinatensystems. Das Gehirn wurde so weit um den Ursprung des Koordinatensystems rotiert, dass die Medianebene die AC-PC-Achse enthielt und vertikal ausgerichtet war (AC-PC-Ebene). Die transversale Ebene hatte ihren Ursprung in gleicher Weise in der vorderen Kommissur und stand orthogonal zur AC-PC-Ebene. Darüber hinaus dienten die äußersten Grenzen des Gehirns ebenfalls als Orientierungspunkte für die Transformation der anatomischen Datensätze auf das Talairachsystem.

Mit Hilfe der Vorverarbeitung der T2*-gewichteten Daten konnten Artefakte, die im Rahmen der fMRT-Untersuchung auftraten, minimiert und die Qualität der Rohdaten verbessert werden. Ziel war ein erhöhter Signal-/Rauschabstand der Daten und damit eine erhöhte statistische Validität der späteren Auswertung.

Der erste Schritt der Vorverarbeitung enthielt eine vom Scanner ausgeführte Bewegungskorrektur. Es folgten weitere Vorverarbeitungsschritte mit Hilfe von Brain Voyager QX 1.10.2, die eine 3D-Bewegungskorrektur (3D motion correction oder realignment), die Korrektur der Erfassungsverzögerung (slice scan time correction) sowie die Basislinienkorrektur (linear trend removal) umfassten. 
3D-Bewegungskorrektur: Damit möglichst keine Bewegungsartefakte das Experiment beeinflussten, wurden während der gesamten MRT-Messzeit die Köpfe der Probanden in der Kopfspule mit weichen Schaumstoffpolstern fixiert. Dennoch konnten kleinste Bewegungen der Probanden dazu führen, dass das Signal eines Ortes im Gehirn zu unterschiedlichen Zeiten der Messung in unterschiedlichen Voxeln abgebildet wurde. Um diese Bewegungsartefakte so klein wie möglich zu halten, wurde die 3-DBewegungskorrektur durchgeführt. Dazu wurde durch Verschiebung entlang der drei Raumrichtungen $x, y$, und $z$ sowie Rotation um diese drei Raumachsen jedes einzelne Bild mit dem ersten Bild der Sequenz abgeglichen und in Deckung gebracht (Jenkinson et al. 2002). Das Protokoll der Bewegungskorrektur diente gleichzeitig der Überprüfung der Qualiät der fMRT-Daten. Es visualisierte alle Bewegungen des Probanden. Bewegungen von mehr als zwei Millimetern von der Ausgangsposition wurden als Ausschlusskriterium für die Messungen benutzt.

Korrektur der Erfassungsverzögerung: Die funktionellen Daten wurden mit EPI-Technik aufgenommen. EPI-Messungen sind Schichtmessungen, bei denen innerhalb der Repetitionszeit Aufnahmen verschiedener Schichten des Gehirns erstellt werden, die zusammen das gesamte Gehirn abbilden. Die Erfassungsverzögerung wird durch eine Interpolation der Intensitätswerte der Bildpunkte auf einen mittleren Referenzzeitpunkt innerhalb der Repetitionszeit korrigiert. Die in dieser Studie verwendete Repetitionszeit von zwei Sekunden war ursächlich dafür, dass die Zeitpunkte der einzelnen Schichtaufnahmen nur geringfügig auseinanderlagen und die Korrektur der Erfassungsverzögerung in Anbetracht der gewählten Testparadigmen mit 36 Sekunden Blockdesign einen eher kleinen Effekt hatte.

Basislinienkorrektur: Während einer fMRT-Messung tritt im Verlauf des Experiments häufig eine Abdrift des Mittelwertes des Signals nach oben oder unten auf. Ursache für diese Basislinienschwäche ist eine rein technisch bedingte und im Vorfeld nicht vollständig kompensierbare Scanner-Abdrift (scanner drifts). Zur Bereinigung des Signals wurde im Rahmen der Vorverarbeitung von fMRT-Daten die Basislinienkorrektur (linear trend removal) mittels Hochpass-Filter durchgeführt (Skudlarski et al. 1999).

Koregistrierung: Die fMRT-Aufnahmen wurden in T2*-Wichtung aufgenommen, die eine relativ geringe räumliche Auflösung besitzt. Im Gegensatz dazu sind die anatomischen T1-gewichteten Aufnahmen höher aufgelöst und verzerrungsärmer. Damit die Aktivierungskarten genauen anatomischen Strukturen und Arealen zugeordnet werden konnten, musste der funktionelle Datensatz mit den zuvor talairach-normalisierten anatomischen Referenzaufnahmen koregistriert werden.

Für die Koregistrierung führte das Program Brainvoyager zunächst eine Ausrichtung der anatomischen und der funktionellen Sequenzen anhand der Positionsparameter des MR-Gerätes, die im Header der Rohdaten hinterlegt waren, durch. Abweichungen 
durch minimale Kopfbewegungen der Probanden wurden manuell korrigiert. Dabei wurden die Grenzen des Gehirns, die Sulci und die Gyri beider Sequenzen entlang der 6 Freiheitsgrade, die schon in der 3D-Bewegungskorrektur erwähnt wurden, verschoben und rotiert und so in Deckung gebracht. Durch diesen Schritt war außerdem eine manuelle Plausibilitäts- und Qualitätskontrolle aller Sequenzen möglich. Die Daten beider Aufnahmetechniken lagen nun im normalisierten Talairachkoordinatensystem vor.

FMRT-Datensätze werden in der Medizin üblicherweise in zwei Dimensionen erstellt. Um eine dreidimensionale Auswertung zu ermöglichen, wurden die funktionellen Daten schließlich von Brainvoyager in einen dreidimensionalen Datensatz transformiert.

\section{6 fMRT-Datenanalyse}

Die vorverarbeiteten Daten der fMRT-Messung konnten nun zu Aktivierungskarten weiterverarbeitet und anschließend ausgewertet werden.

Funktionelle Aktivierungskarten zeigen die individuelle neurologische Antwort eines Probanden auf kognitive Reize. Sie sind statistische Korrelationskarten, die diejenigen Orte im Gehirn anzeigen, die auf einen Stimulus hin angeregt werden.

Zur Erstellung der Aktivierungskarten wurde das allgemeine lineare Modell auf der Basis des Stimulationsparadigmas sowie der hämodynamischen Antwortfunktion auf alle Bildpunkte der vorverarbeiteten funktionellen T2*-Daten angewendet.

Das allgemeine lineare Modell vergleicht eine idealisierte Referenzfunktion mit der tatsächlichen Datenzeitreihe der Aktivierung in jedem einzelnen Bildpunkt. Der Verlauf der Referenzfunktion wird von der zeitlichen Abfolge des Stimulationsparadigmas bestimmt und beschreibt die erwartete Antwort des Gehirns auf einen Stimulus. Je besser die Daten eines Bildpunktes mit der Referenzfunktion korrelieren, desto wahrscheinlicher ist die Aktivierung in diesem Bildpunkt durch den Stimulus bedingt und nicht zufällig. Aus dem Vergleich mit dem allgemeinen linearen Modell resultiert eine Darstellung des Korrelationskoeffizienten im dreidimensionalen Datensatz mit einer festgelegten Skalierung.

Da das allgemeine lineare Modell auf jeden einzelnen Bildpunkt des Gehirns angewendet wurde, musste das Problem der multiplen Vergleiche (multiple comparisons problem) korrigiert werden. Da jeder Bildpunkt mit einer individuellen Irrtumswahrscheinlichkeit von 0,05 getestet wurde, multiplizierte sich die versuchsbezogene Irrtumswahrscheinlichkeit um die Anzahl der getesteten Bildpunkte. Dieses führte zu einer Alphafehler-Kumulierung.

Ein etabliertes Konzept zur Korrektur der multiplen Vergleiche bei fMRT-Daten ist die Anwendung der Falscherkennungsrate (FDR: false discovery rate) (Benjamini und 
Hochberg 1995; Genovese et al. 2002). Dieses statistische Konzept kontrolliert die Anzahl der falsch positiven Bildpunkte innerhalb der Gruppe der signifikant aktivierten Bildpunkte und ist daher ideal für fMRT-Daten. Die Methode der Falscherkennungsrate wird von Brainvoyager QX zur Korrektur der multiplen Vergleiche angewendet und ist im Bereich der fMRT-Forschung etabliert.

Die Referenzfunktion orientierte sich an dem neurophysiologischen Testparadigma im Blockdesign (Abbildung 6a) und wurde mit der HRF moduliert (Abbildung 6b). Diese zeigt nicht die direkte neuronale Aktivität des Gehirns auf einen Stimulus, sondern entspricht der mit Latenz zum Stimulus auftretenden hämodynamischen Antwort. Die HRF beschreibt, wie sich das BOLD-Signal theoretisch auf einen Stimulus hin verhält, und zeigt einen allmählichen Anstieg bis zum Maximum, gefolgt von einem langsameren Abfall bis zur Nullinie und schließlich einem leichten Unterschreiten der Nulllinie, bis sie wieder erreicht wird (Friston et al. 1994; Logothetis et al. 2001; Ogawa et al. 1990).

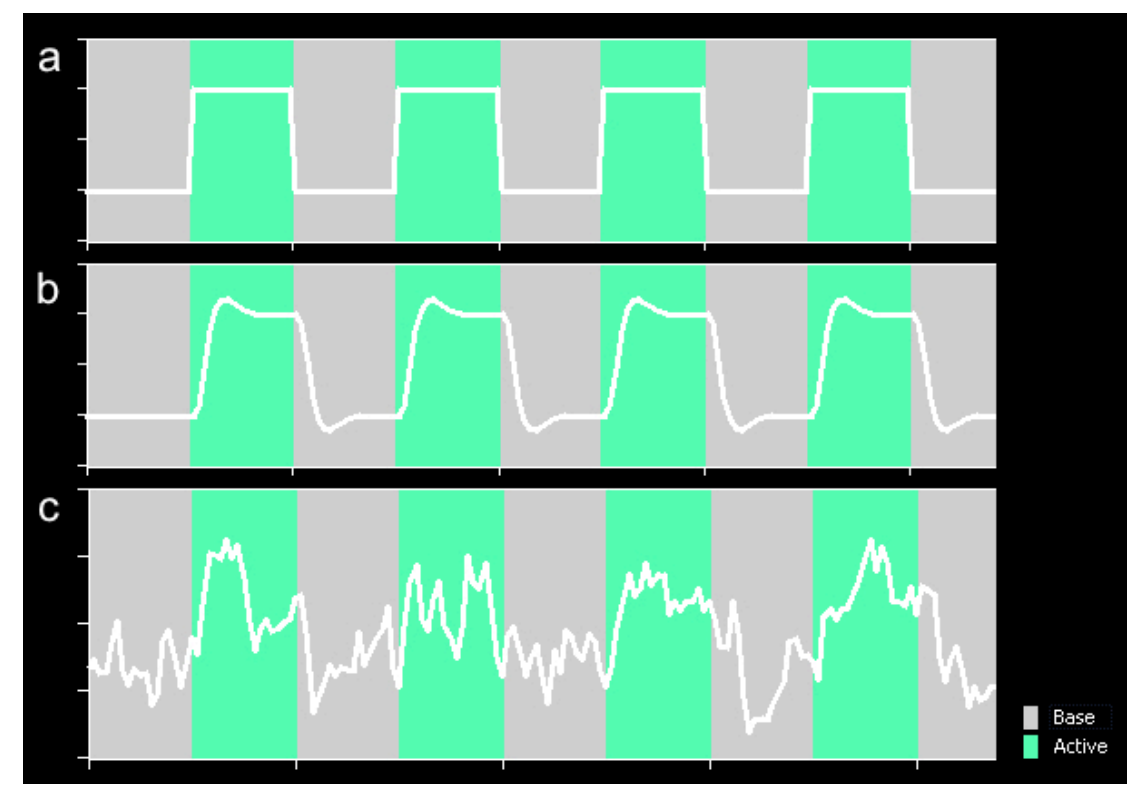

Abbildung 6:

a) Blockdesign des Testparadigmas

b) Modulation des Blockdesigns mit der HRF

Die erstellten statistischen Aktivierungskarten zeigten nun Aktivierungscluster, die der individuellen Ausprägung der Hirnaktivität während des fMRT-Experiments entsprachen.

Aufgrund der individuellen Ausprägung wurden die statistischen Grenzen der Aktivierungskarten für jeden Probanden entsprechend angepasst, so dass Signifikanzniveaus von $p<0,047192$ erreicht wurden (Tabelle 3 bis 21). Diese statistischen Schwellen blieben im weiteren Verlauf der Auswertung individuell konstant. Innerhalb der Aktivierungscluster konnten anschließend die ROls für die statistische Auswertung bestimmt werden.

ROls können, je nach Fragestellung, anhand anatomischer Orientierungspunkte (anatomische ROI) oder auf Basis funktioneller Aktivierungskarten (funktionelle ROI) 
definiert werden. Anatomische ROIs enthalten alle Voxel einer bestimmten anatomischen Struktur, während funktionelle ROls aus einem Verband (cluster) von aktivierten Voxeln während eines Experimentes bestehen.

In dieser Studie wurde eine Kombination dieser beiden Verfahren angewendet. Den Ausgangspunkt bildeten zunächst die Talairachkoordinaten des Broca-Areals und des Wernicke-Areals der dominanten Hemisphäre, die mit Hilfe des TalairachClient 2.4 (Lancaster et al. 1997; 2000) in den funktionellen Aktivierungskarten identifiziert wurden.

Um der individuellen funktionellen Aktivierung der Probanden gerecht zu werden, wurden diejenigen zusammenhängenden Bildpunktcluster, die mit diesen anatomischen Orientierungspunkten korrelierten, als ROI gewählt.

Analog dazu wurden auch in der nichtdominanten Hemisphäre Aktivitätscluster als ROIs definiert. Hier dienten als Orientierungspunkte die Kortexregionen, die räumlich der Lokalisation des Broca- und Wernicke-Areals in der dominanten Hemisphäre entsprachen. Die Aktivitätscluster, die mit diesen anatomischen Orientierungspunkten korrelierten, wurden ebenfalls als ROI definiert (Abbildung 7 und 8).
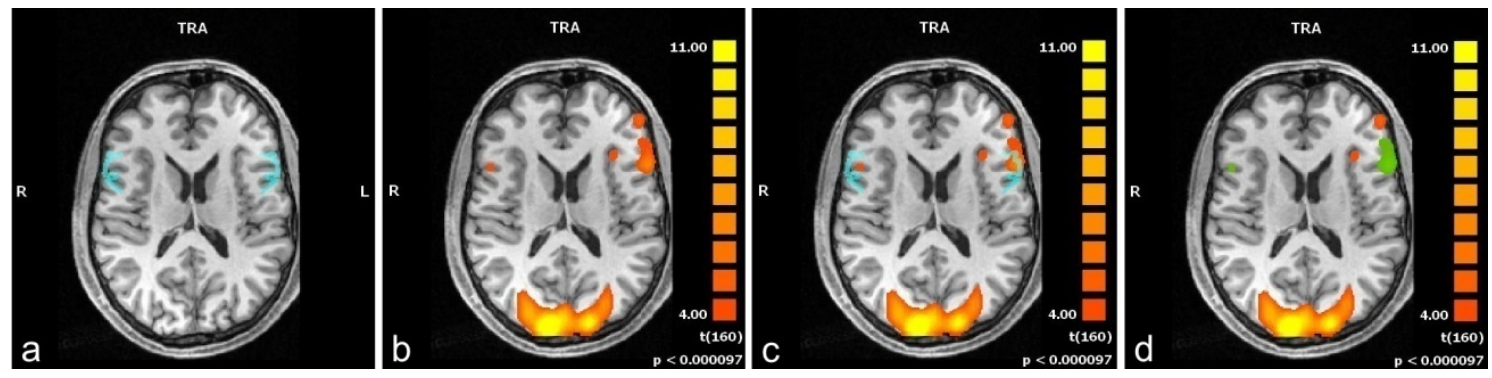

Abbildung 7: Bestimmung der funktionellen ROls im Bereich des Broca-Areals am Beispiel des Sprachtests „Satzgenerierung“. a) Anatomische Lokalisation der BA 44 und 45 (hellblau). b) Funktionelle Aktivierungskarte. c) Kombination der anatomischen Lage der Broca-Region und der funktionellen Aktivierungskarte. d) Definierte ROI „Satz Broca“ (grün).
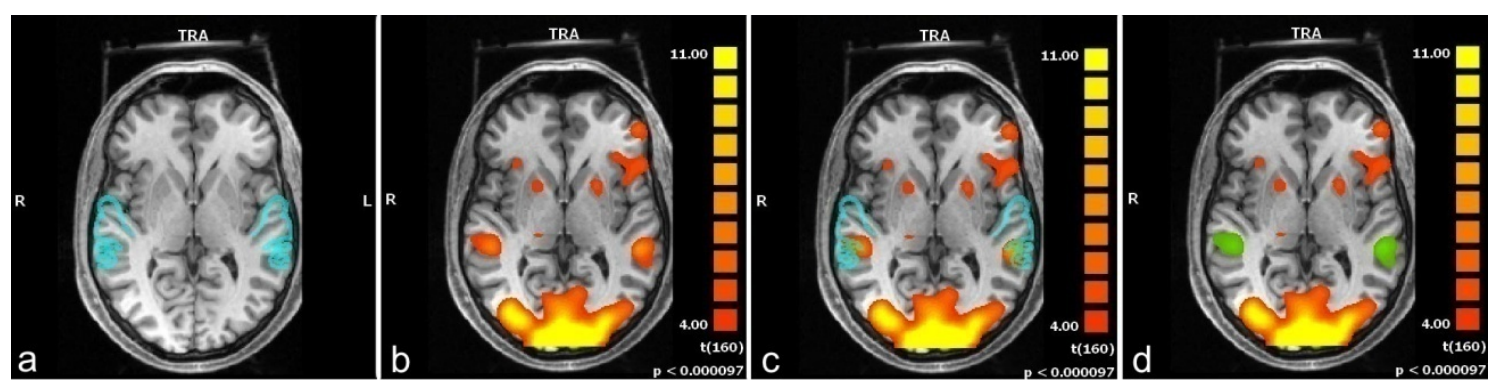

Abbildung 8: Bestimmung der funktionellen ROls im Bereich des Wernicke-Areals am Beispiel des Sprachtests "Satztgenerierung“. a) Anatomische Lokalisation der BA 22 (hellblau). b) Funktionelle Aktivierungskarte. c) Kombination der anatomischen Lage der Wernicke-Region und der funktionellen Aktivierungskarte. d) Definierte ROI „Satz Wernicke“ (grün).

So entstanden pro Proband im Sprachtest „Wortgenerierung“ die ROls „Wort Broca links“, „Wort Broca rechts“ sowie „Wort Wernicke links“ und „Wort Wernicke rechts“. Im 
zweiten Testparadigma „Satzgenerierung“ wurden die ROIs „Satz Broca links“, „Satz Broca rechts" sowie "Satz Wernicke links" und "Satz Wernicke rechts" definiert. Für jede ROI wurden der Aktivitätsschwerpunkt, die Anzahl der signifikant aktivierten Voxel und das Signifikanzniveau ( $p$ ) dokumentiert. Die Anzahl der signifikant aktivierten Bildpunkte innerhalb einer ROI diente als Maß für die Quantität der Aktivierung und wurde zur Berechnung eines Lateralitätsindex herangezogen.

\section{7 fMRT-Lateralitätsindex}

Bestimmte kognitive Leistungen, wie z. B. Sprache, sind in den Hemisphären unterschiedlich stark ausgeprägt, wobei eine Hemisphäre in Bezug auf diese kognitive Funktion dominant ist (vergleiche Kapitel 1.2.1).

Die relative Beteiligung der Hemisphären an einer kognitiven Leistung kann durch einen Lateralitätsindex (LI) beschrieben werden. Dieser gibt Auskunft über die Hemisphärendominanz und den Grad der Lateralisierung (Jansen et al. 2006).

LIs für die fMRT-Daten wurden für jeden Probanden und jede Region von Interesse nach folgender Formel berechnet:

$L I=\left(L_{f M R I}-R_{f M R I}\right) /\left(L_{f M R I}+R_{f M R I}\right)$

$\mathrm{L}_{\mathrm{fMRI}}$ und $\mathrm{R}_{\mathrm{fMRI}}$ bezeichneten die Anzahl der signifikant aktivierten Bildpunkte einer ROI in der linken $\left(\mathrm{L}_{\mathrm{fMRI}}\right)$ und der zugehörigen $\mathrm{ROI}$ der rechten $\left(\mathrm{R}_{\mathrm{fMRI}}\right)$ Hemisphäre. Ein $\mathrm{LI} \geq$ 0,1 wurde als linkslateralisierte Aktivierung und ein $\mathrm{LI} \leq-0,1$ wurde als rechtslateralisierte Aktivierung gewertet. Probanden mit einem Lateralitätsindex von $-0,1<\mathrm{LI}<0,1$ zeigten eine funktionelle Kodominanz beider Hemisphären (Lurito und Dzemidzic 2001).

So ergaben sich für den Sprachtest „Satzgenerierung“ die Lateralitätsindizes „LI Satz Broca“ und „LI Satz Wernicke“ sowie für den Sprachtest „Wortgenerierung" die Lateralitätsindizes „LI Wort Broca“ und „LI Wort Wernicke“.

\subsection{DTI-Datenverarbeitung}

Die erhobenen DTI-Aufnahmen lieferten Informationen über die weiße Substanz des Gehirns, so dass neuroanatomische Grundlagen der funktionellen Sprachlateralisierung untersucht werden konnten. Die fraktionelle Anisotropie (FA) beschreibt das Diffusionsverhalten in einem Voxel. Eine hohe gerichtete Diffusion, wie sie z. B. in Faserbündeln mit vielen parallelen Diffusionsbarrieren vorkommt, geht mit einer hohen FA einher. Auf diese Weise konnten mit Hilfe der FA Aussagen über die Struktur der weißen Substanz objektiviert werden (siehe Kapitel 1.4.4.2). 
Die DTI-Aufnahmen wurden analog zu den fMRT-Aufnahmen mit Brain Voyager QX 1.10.2 (Brain Innovation, Maastricht, The Netherlands) aufbereitet (Goebel et al. 2006).

Rohdaten:

- 3 x DTI-Aufnahmen, Ebenenauflösung 2,2 x 2,2 $\mathrm{mm}^{2}$, Schichtdicke 2,2mm, STEAM-Sequenz, 25 Richtungen, 38 Schichten

Zunächst wurde jede der drei DTI-Sequenzen mit der räumlich höher aufgelösten T1gewichteten anatomischen Referenzsequenz koregistriert. Die Koregistrierung wurde analog zur beschriebenen Koregistrierung der fMRT-Daten mit den anatomischen Daten in Abschnitt 2.4 durchgeführt: nach der mathematischen Ausrichtung anhand der Positionsparameter im Header der Rohdaten durch das Programm Brainvoyager wurden leichte Kopfbewegungen der Probanden zwischen den Sequenzen zusätzlich manuell korrigiert. Die Grenzen des Gehirns, die Sulci und die Gyri beider Sequenzen wurden entlang der 6 Freiheitsgrade verschoben und rotiert und dadurch in Deckung gebracht. Durch diesen Schritt erfolgte erneut eine zusätzliche manuelle Kontrolle aller Sequenzen. Die Daten beider Aufnahmetechniken lagen nun im normalisierten Talairachkoordinatensystem vor, so dass in der späteren statistischen DTIDatenanalyse eine genaue Zuordnung der FA-Werte zu anatomischen Strukturen und damit auch zu den funktionellen Bilddaten möglich war.

Das Programm Matlab Version 7.4 (The MathWorks Inc.) verarbeitete anschließend die drei vorbereiteten diffusionsgewichteten Datensätze zu einem gemittelten Datensatz. Durch die Mittelung dieser Sequenzen erfolgte eine Verbesserung des SNR der DTIDaten. Der gemittelte DTI-Datensatz wurde anschließend in Brainvoyager QX zur weiteren Auswertung verwendet.

Aus den diffusionsgewichteten Daten wurde die FA jedes Voxels berechnet. Die Eigenschaften des dreidimensionalen Diffusionsverhaltens von Wasserstoffmolekülen wurden auf diese Weise in einer FA-Karte abstrahiert dargestellt. Dadurch konnten das Ausmaß der Anisotropie berechnet und Aussagen über die Mikrostruktur der weißen Substanz getroffen werden (Mori und van Zijl 2002).

\subsection{DTI-Datenanalyse}

Durch Verknüpfung der FA-Karte mit dem anatomischen 3D-Datensatz konnten die ROls, die im Rahmen der Auswertung der fMRT-Datensätze bereits in der linken und der rechten Hemisphäre definiert wurden, auch in der FA-Karte eingeblendet werden. Für jeden Voxel dieser ROls konnte anschließend der mittlere Wert der FA in der mit der funktionellen ROI korrelierenden weißen Substanz bestimmt werden. Die Talairachkoordinaten des Voxels mit der höchsten FA wurden bestimmt. Diese Koordinaten dienten als Mittelpunkt einer strukturellen ROI mit einer Ausdehnung von 3 
x 3 Voxeln, die in den FA-Karten definiert wurde (Abbildung 9). Die strukturellen ROIs wurden analog zu den funktionellen ROls beider Hemisphären, die als Ausgangspunkt für die Bestimmung der strukturellen ROls dienten, wie folgt benannt: „Wort Broca links“, „Wort Broca rechts“, „Wort Wernicke links“, „Wort Wernicke rechts“, „Satz Broca links“, „Satz Broca rechts“, „Satz Wernicke links“ und „Satz Wernicke rechts“. Anhand der FA-Werte dieser strukturellen ROls wurde ein Asymmetrieindex analog zum Lateralitätsindex der funktionellen Daten errechnet.
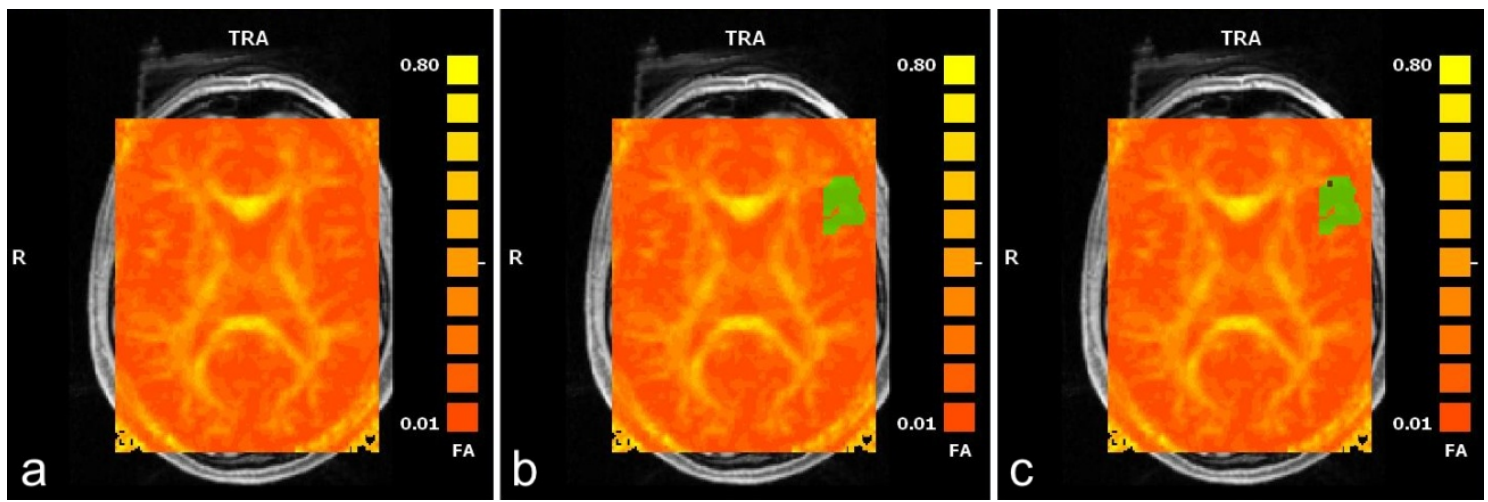

Abbildung 9: Beispielhafte Bestimmung der strukturellen ROI „Satz Broca links“. a) FA-Karte. b) Lokalisation der funktionellen ROI „Satz Broca links“ (grün). c) Lokalisation der strukturellen ROI „Satz Broca links“ (schwarz). In gleicher Weise diente die Einblendung der funktionellen ROls der rechten Hemisphäre der Bestimmung der strukturellen ROls der rechten Hemisphäre.

\subsection{FA-Asymmetrieindex}

Auch die an der Sprachverarbeitung beteiligte weiße Substanz zeigt eine strukturelle Asymmetrie im MRT (Good et al. 2001), die durch einen Asymmetrieindex (Al) beschrieben und quantifiziert werden kann.

Für jeden Probanden und jede strukturelle ROI wurde auf Grundlage des mittleren Wertes der FA ein Al nach folgender Formel berechnet:

$A I=\left(L_{F A}-R_{F A}\right) /\left(L_{F A}+R_{F A}\right)$

$L_{F A}$ und $R_{F A}$ bezeichneten den mittleren Wert der FA einer strukturellen $R O I$ in der linken $\left(L_{F A}\right)$ oder der zugehörigen rechten $\left(R_{F A}\right)$ Hemisphäre.

Ein $\mathrm{Al} \geq 0,1$ wurde als linksgerichtete Asymmetrie mit linkshemisphärisch höherer FA und ein $A l \leq-0,1$ als rechtsgerichtete Asymmetrie mit rechtshemisphärisch höherer FA bewertet. Probanden mit einem Asymmetrieindex von $-0,1<\mathrm{Al}<0,1$ zeigten eine symmetrische Verteilung der FA in beiden Hemisphären (Vernooij et al. 2007).

So ergaben sich die Asymmetrieindizes „Al Satz Broca“ und „Al Satz Wernicke“ für die strukturellen ROIs der FA-Karten, die auf Grundlage der funktionellen Datenauswertung des Sprachtests „Satzgenerierung" erstellt wurden. Die 
Asymmetrieindizes „Al Wort Broca“ und „Al Wort Wernicke“ beschrieben die FA der strukturellen ROls, die auf Grundlage der funktionellen ROls des Sprachtests „Wortgenerierung“ erstellt wurden.

\subsection{Statistische Auswertung}

Die statistische Auswertung wurde mit Statistica ${ }^{\circledR}$ Version 9 (StatSoft, Inc. 1984-2009, Tulsa, USA) sowie Microsoft $®$ Office Excel $₫ 2007$ durchgeführt.

$\mathrm{Da}$ das verwendete Testparadigma zur Bestimmung der funktionellen Sprachlateralisierung in vorangegangenen Studien keine Normalverteilung der Sprachlateralisierung zeigte (Stippich et al. 2003), musste auch von nicht normalverteilten LIs in der vorliegenden Arbeit ausgegangen werden. In der statistischen Auswertung wurden daher nichtparametrische Tests (Mann-Whitney-UTest und Vorzeichentest) angewendet. Sie setzten für die Berechnung keine Normalverteilung der Daten voraus und konnten ohne eine Annahme über die Verteilung angewendet werden.

Die funktionellen Lls und die strukturellen Als wurden mit dem Mann-Whitney-U-Test auf signifikante Unterschiede zwischen dem Kontrollkollektiv und dem Kollektiv der Patienten mit fokaler Epilepsie geprüft. In der Gruppe der Patienten mit fokaler Epilepsie wurde zudem eine Untergruppe mit denjenigen Patienten gebildet, die eine wahrscheinlich linkshemisphärische Epileptogenese aufwiesen (Gruppe E links). Diese Untergruppe wurde in gleicher Weise mit dem Kontrollkollektiv verglichen. Es wurde untersucht, ob die Ergebnisse bezüglich der Verteilung bei einem homogeneren Patientenkollektiv ihre Gültigkeit behielten.

Die Gleichsinnigkeit der LIs der fMRT-Messungen und der Als der DTI-Messungen bezüglich der Hemisphärenangabe innerhalb einer Gruppe wurde mit dem Vorzeichentest überprüft.

Bei allen Tests wurden p-Werte unter 0,05 als statistisch signifikant bewertet.

\subsubsection{MANN-WhITNEY-U-TEST}

Der Mann-Whitney-U-Test untersucht zwei unabhängige Gruppen (Kontrollkollektiv und Patientenkollektiv) mit nicht normalverteilten kontinuierlichen Variablen (LI und Al).

Für jede der im Abschnitt 2.5 definierten ROls („Wortgenerierung“: Wort Broca links, Wort Broca rechts, Wort Wernicke links, Wort Wernicke rechts; „Satzgenerierung“: Satz Broca links, Satz Broca rechts, Satz Wernicke links, Satz Wernicke rechts) wurden die LIs der fMRT-Daten des Kollektivs der Patienten mit fokaler Epilepsie gegen die 
entsprechenden LIs der fMRT-Daten des Kontrollkollektivs auf folgende zwei Hypothesen getestet:

Nullhypothese $(\mathrm{H} 0)$ : Es bestand kein Unterschied hinsichtlich der Verteilung der LIs der fMRT-Daten zwischen der Gruppe der Kontrollprobanden und der Gruppe der Patienten mit fokaler Epilepsie.

Alternativhypothese $(\mathrm{H} 1)$ : Die Verteilung der LIs der fMRT-Daten unterschied sich in den beiden untersuchten Gruppen (Kontrollprobanden und Patienten mit fokaler Epilepsie).

Analog dazu wurden die Als der DTI-Daten des Patientenkollektivs gegen die entsprechenden Als der DTI-Daten des Kontrollkollektivs auf folgende zwei Hypothesen getestet:

Nullhypothese $(\mathrm{H} 0)$ : Es bestand kein Unterschied hinsichtlich der Verteilung der Als der DTI-Daten zwischen der Gruppe der Kontrollprobanden und der Gruppe der Patienten mit fokaler Epilepsie.

Alternativhypothese (H1): Die Verteilung der Als der DTI-Daten unterschied sich in den beiden untersuchten Gruppen (Kontrollprobanden und Patienten mit fokaler Epilepsie).

Aufgrund des $5 \%$-Signifikanzniveaus $(\alpha=0,05)$ konnte die Nullhypothese zu einem Fehlerniveau von $5 \%$ abgelehnt werden, wenn $p \leq \alpha$ war.

Wenn $p>a$ war, konnte zu einem Fehlerniveau von 5\% keine Signifikanz festgestellt werden und die Nullhypothese durfte nicht abgelehnt werden.

Zur Prüfung, ob sich die Verteilung der Lls und Als anders verhielt, wenn ein homogeneres Patientenkollektiv mit ausschließlich linkshemisphärischer Epileptogenese untersucht wurde, erfolgte in gleicher Weise der Vergleich des Kontrollkollektivs N mit dem Patientenkollektiv E links.

\subsubsection{VORZEICHENTEST (NICHTPARAMETRISCH)}

Der Vorzeichentest untersucht gepaarte Gruppen (Kontrollkollektiv N oder Patientenkollektive $\mathrm{E}$ und $\mathrm{E}$ links) mit nicht normalverteilten kontinuierlichen Variablen.

Für jede ROI wurde innerhalb einer Gruppe der LI der fMRT-Daten gegen den entsprechenden AI der DTI-Daten auf folgende zwei Hypothesen getestet:

Nullhypothese $(\mathrm{HO})$ : Es bestand kein Unterschied hinsichtlich der Verteilung der LIs der fMRT-Daten und der Als der DTI-Daten innerhalb einer Gruppe (Kontrollprobanden oder Patienten mit fokaler Epilepsie). 
Alternativhypothese $(\mathrm{H} 1)$ : Die Verteilung der LIs der fMRT-Daten und der Als der DTIDaten unterschied sich innerhalb einer Gruppen (Kontrollprobanden oder Patienten mit fokaler Epilepsie).

Aufgrund des 5\%-Signifikanzniveaus $(\alpha=0,05)$ konnte die Nullhypothese zu einem Fehlerniveau von $5 \%$ abgelehnt werden, wenn $p \leq \alpha$ war.

Wenn $p>a$ war, konnte zu einem Fehlerniveau von 5\% keine Signifikanz festgestellt werden und die Nullhypothese durfte nicht abgelehnt werden. 


\section{ERgebNISSE}

\subsection{Händigkeit}

Mit Hilfe des Edinburgh-Händigkeitsinventars (Oldfield 1971) wurde bei allen Probanden zu Beginn der MRT-Studie die Händigkeit bestimmt. Die errechneten LIs zeigten, dass alle Probanden des Patientenkollektivs und des Kontrollkollektivs Rechtshänder waren.

Im Kontrollkollektiv lagen die LIs zwischen +60 und +100 . Alle Probanden des Kontrollkollektivs konnten daher als Rechtshänder klassifiziert werden (siehe Kapitel 2.2). 16 der 23 Kontrollprobanden erhielten einen $\mathrm{LI}$ von +100 , darunter vier Frauen und zwölf Männer. Die restlichen sieben Probanden, zwei Frauen und fünf Männer, lagen in einem Bereich von +60 bis +87 .

In der Gruppe der Patienten mit fokaler Epilepsie lagen die LIs zwischen +50 und +100. Daher konnten auch alle Probanden des Patientenkollektivs als Rechtshänder klassifiziert werden. Sieben der zwölf Patienten mit fokaler Epilepsie erhielten einen LI von +100 , drei Frauen und vier Männer. Die restlichen männlichen Patienten lagen in einem Bereich von +50 bis +80 (Tabelle 1 ).

\section{2 fMRT-Ergebnisse}

Alle Probanden zeigten statistisch signifikante Aktivierungen in mindestens einer Hemisphäre während eines oder beider Sprachtests in den sprachassoziierten frontalen und parietotemporalen Kortexarealen.

Im Folgenden wurde das Ausmaß der Aktivität innerhalb der ROIs ausgewertet und daraus die LIs der fMRT-Daten berechnet. Die Ergebnisse der beiden untersuchten Probandengruppen wurden anschließend miteinander verglichen.

Die Auswertung der fMRT-Daten zeigte im Broca-Areal während beider Sprachtests, dass sich die Verteilung der LIs innerhalb der untersuchten Gruppen signifikant voneinander unterschied. Auch der Vergleich des Kontrollkollektivs mit den Patienten mit ausschließlich linkshemisphärischer Epileptogenese zeigte eine signifikant unterschiedliche Verteilung der LIs innerhalb der Gruppen. Die Nullhypothese konnte in dieser Region mit den in dieser Studie verwendeten Tests abgelehnt werden.

Für das Wernicke-Areal hingegen konnte die Auswertung während keines der beiden Sprachtests signifikante Unterschiede in der Verteilung der LIs innerhalb der beiden Gruppen nachweisen. Dieses änderte sich auch nicht im Vergleich des Kontrollkollektivs mit den Patienten, die eine ausschließlich linkshemisphärische 
Epileptogenese aufwiesen. Die Nullhypothese durfte in der Wernicke-Region mit den verwendeten Sprachtests somit nicht abgelehnt werden (Tabelle 27 und 28).

\subsubsection{SPRAChtest „WORTgenerierUng“ in DER Broca-Region}

22 der 23 Probanden des Kontrollkollektivs zeigten während des Sprachtests „Wortgenerierung“ signifikante Aktivierung im Bereich der Broca-Region in mindestens einer der beiden Hemisphären. Bei einer Versuchsperson des Kontrollkollektivs konnte weder in der linken noch in der rechten Hemisphäre eine signifikante Aktivierung während dieses Abschnitts des Experiments ermittelt werden.

Bei der Auswertung des Kollektivs der Patienten mit fokaler Epilepsie zeigten alle zwölf Patienten in den definierten ROls im Gebiet der Broca-Region während des Sprachtests „Wortgenerierung“ eine statistisch signifikante kortikale Aktivierung in mindestens einer der beiden Hemisphären (Tabelle 3 bis 6).

\subsubsection{BROCA-REGION LINKS IM KOLLEKTIV N}

Bei der Auswertung der definierten ROls im Gebiet der Broca-Region der linken Hemisphäre zeigten 22 der 23 Probanden des Kontrollkollektivs eine statistisch signifikante kortikale Aktivierung im Zusammenhang mit dem Sprachtest „Wortgenerierung“. Lediglich Proband 18 zeigte keine Aktivierung in der linken Hemisphäre während dieses Tests.

Anatomische Aktivitätsschwerpunkte der Probanden lagen bei 16 Probanden im Gyrus frontalis inferior, bei zwei Probanden im Gyrus frontalis medialis und bei vier Probanden in der Insel.

Bezogen auf die Brodmann-Areale ergab sich somit bei 10 Probanden und damit am häufigsten eine Lokalisation der Aktivitätsschwerpunkte im BA 45. Bei sechs Probanden lagen die Aktivitätsschwerpunkte im BA 13, bei zwei Probanden im BA 44, bei weiteren zwei Probanden im BA 46, bei einem Probanden im BA 47 und bei einem weiteren Probanden im BA 9 (Tabelle 3).

\subsubsection{BROCA-REGION RECHTS IM KOLLEKTIV N}

13 der 23 Probanden des Kontrollkollektivs zeigten in den definierten ROls im Gebiet der Broca-Region der rechten Hemisphäre eine statistisch signifikante kortikale Aktivierung im Zusammenhang mit dem Sprachtest „Wortgenerierung“. Zehn Probanden zeigten keine rechtshemisphärische Aktivierung während dieses Tests.

Die anatomischen Aktivitätsschwerpunkte der Probanden, die in der rechten Hemisphäre signifikante Aktivität im Broca-Areal zeigten, lagen bei sieben Probanden in der Inselrinde, bei vier Probanden im Gyrus frontalis inferior, bei einem Probanden im Gyrus frontalis medialis und bei einem Probanden im Gyrus praecentralis. 
Bezogen auf die Brodmann-Areale lagen die Aktivitätsschwerpunkte am häufigsten im BA 13. Hier zeigten acht der Probanden signifikante Aktivität. Bei zwei weiteren Probanden lagen die Aktivitätsschwerpunkte im BA 44 und bei jeweils einem Probanden im BA 45, 46 und 47 (Tabelle 4).

\subsubsection{BROCA-REGION LINKS IM KOLLEKTIV E}

Die Auswertung des Kollektivs der Patienten mit fokaler Epilepsie zeigte bei allen zwölf Patienten eine statistisch signifikante kortikale Aktivierung in den definierten ROls im Gebiet der Broca-Region der linken Hemisphäre während des Sprachtests "Wortgenerierung“.

Bei fünf Patienten lagen die anatomischen Aktivitätsschwerpunkte in der Inselrinde, bei vier Patienten im Gyrus frontalis inferior, bei zwei Patienten im Gyrus praecentralis und bei einem Patienten im Gyrus frontalis medialis.

Bezogen auf die Brodmann-Areale ergab sich somit, dass bei sechs Patienten die Aktivitätsschwerpunkte im BA 13 lagen. Dieses Areal war damit am häufigsten vertreten. Bei drei weiteren Patienten lagen die Aktivitätsschwerpunkte im BA 45 und bei jeweils einem Patienten im BA 6, BA 44 und BA 46 (Tabelle 5).

\subsubsection{BROCA-REGION RECHTS IM KOLLEKTIV E}

Bei der Auswertung der kortikalen Aktivierung in der rechten Hemisphäre während des Sprachtests „Wortgenerierung“ zeigten elf der zwölf Patienten mit fokaler Epilepsie in den definierten ROls im Gebiet der Broca-Region eine statistisch signifikante Aktivierung. Proband 31 zeigte keine Aktivierung in der rechten Hemisphäre.

Die anatomischen Aktivitätsschwerpunkte lagen bei fünf Patienten im Gyrus frontalis inferior, bei vier Patienten in der Inselrinde und bei jeweils einem Patienten im Gyrus frontalis medialis und im Claustrum.

Bei neun der elf Patienten, die kortikale Aktivierung in der rechten Hemisphäre zeigten, konnten den Aktivitätsschwerpunkten auch Brodmann-Areale zugeordnet werden. Bei drei Patienten lagen die Aktivitätsschwerpunkte im BA 13, bei jeweils zwei Patienten im BA 44 und 45 und bei jeweils einem Patienten im BA 46 und 47. Den Probanden 27 und 32 mit Aktivierung im Claustrum und in der Insel konnte kein BA zugeordnet werden (Tabelle 6).

\subsubsection{VERGLEICH DER LIS IN DER BROCA-REGION WÄHREND DES SPRACHTESTS "WORTGENERIERUNG“"}

Mit Hilfe der Anzahl der signifikant aktivierten Voxel einer ROI in der linken und der zugehörigen ROI in der rechten Hemisphäre wurde für jeden Probanden der LI für die funktionelle Aktivierung innerhalb der Broca-Region während des Sprachtests „Wortgenerierung“ berechnet. 
Die LIs der Gruppe der Patienten mit fokaler Epilepsie wurden anschließend mit denen des Kontrollkollektivs verglichen. Hinsichtlich der Aktivierung in den definierten ROls in der Broca-Region während des Sprachtests "Wortgenerierung“ konnte für 22 der 23 Probanden ein LI berechnet werden. Proband 18 zeigte während des gesamten Test weder Aktivierung in der linken noch in der rechten Hemisphäre. Von den übrigen Probanden zeigten 20 (87\%) eine linksgerichtete Lateralisierung und zwei (9\%) eine rechtsgerichtete Lateralisierung.

In der Gruppe der Patienten mit fokaler Epilepsie konnte für jeden der zwölf Patienten ein LI berechnet werden. Acht Patienten (67\%) dieses Kollektivs zeigten eine Lateralisierung nach links und drei Patienten (25\%) zeigten eine Lateralisierung nach rechts. Ein Patient (8\%) zeigte eine symmetrische Aktivierung (Tabelle 7).

Bei dem Vergleich der LIs beider Gruppen umfassten die linksgerichteten LIs der Kontrollgruppe einen Wertebereich von 0,146 bis 1 mit einem Median von 0,915. Dieser Median lag über dem der Patienten mit fokaler Epilepsie. Die linksgerichteten LIs der Patientengruppe umfassten einen Wertebereich von 0,164 bis 1 mit einem Median von 0,638.

Die rechtsgerichteten LIs der Kontrollgruppe umfassten einen Wertebereich von -0,346 bis -0,137 mit einem Median von -0,242. Die rechtsgerichteten LIs der Patienten mit fokaler Epilepsie umfassten einen Wertebereich von -0,76 bis -0,138 mit einem Median von $-0,169$, der damit über dem Median der Normalprobanden lag.

In der Kontrollgruppe zeigten alle Probanden entweder eine rechtsgerichtete oder eine linksgerichtete Lateralisierung. In der Gruppe der Patienten mit fokaler Epilepsie hingegen wies ein Proband eine symmetrische Aktivierung mit einem LI von 0,006 auf (Tabelle 23).

Mit Hilfe des Mann-Whitney-U-Tests wurden die funktionellen LIs beider Gruppen hinsichtlich ihrer Verteilung innerhalb der Gruppe der Kontrollprobanden und der Gruppe der Patienten mit fokaler Epilepsie untersucht. Die statistische Auswertung ergab, dass sich die funktionelle Lateralisierung des Patientenkollektivs von der des Kontrollkollektivs im Bereich des Broca-Areals während des Sprachtests "Wortgenerierung" signifikant unterschied $(p=0,041)$ (Tabelle 27). Auch die Untersuchung der Verteilung der LIs innerhalb der Gruppe der Kontrollprobanden und der Gruppe mit ausschließlich linkshemisphärischer Epileptogenese zeigte für diese ROI einen signifikanten Unterschied $(p=0,018)$ (Tabelle 28).

\subsubsection{Sprachtest „Wortgenerierung“ IN DER Wernicke-Region}

Alle 23 Probanden des Kontrollkollektivs zeigten während des Sprachtests „Wortgenerierung“ eine signifikante kortikale Aktivierung in den definierten ROls im Bereich der Wernicke-Region in mindestens einer der beiden Hemisphären. 
Ebenso konnte bei allen zwölf Patienten mit fokaler Epilepsie in den definierten ROls im Gebiet der Wernicke-Region während dieses Sprachtests statistisch signifikante Aktivierung in mindestens einer der beiden Hemisphären nachgewiesen werden (Tabelle 8 bis 11).

\subsubsection{WERNICKE-REgION LINKS IM KOLLEKTIV N}

Bei 21 der 23 Probanden des Kontrollkollektivs konnte im Gebiet der Wernicke-Region der linken Hemisphäre statistisch signifikante kortikale Aktivierung im Zusammenhang mit dem Sprachtest „Wortgenerierung“ nachgewiesen werden. Zwei Probanden zeigten keine linkshemisphärische Aktivierung während dieses Tests.

Die anatomischen Aktivitätsschwerpunkte der Probanden mit signifikanter Aktivierung lagen bei elf Probanden im Gyrus temporalis superior, bei acht Probanden im Gyrus temporalis medialis und bei einem Probanden in der Insel.

Bezogen auf die Brodmann-Areale lagen die Aktivitätsschwerpunkte am häufigsten im BA 22. Hier zeigten 10 der Probanden Aktivierung. Bei vier Probanden lagen die Aktivitätsschwerpunkte im BA 42 und bei jeweils zwei Probanden im BA 21 und 41. Den Aktivitätsschwerpunkten der Probanden 6 und 7 konnte kein BA zugordnet werden (Tabelle 8).

\subsubsection{WERnICKE-REgION RECHTS IM KOLLEKTIV N}

Die Auswertung der definierten ROls im Gebiet der Wernicke-Region der rechten Hemisphäre zeigte bei 13 der 23 Probanden des Kontrollkollektivs statistisch signifikante kortikale Aktivierung im Zusammenhang mit dem Sprachtest „Wortgenerierung“. Zehn Probanden zeigten keine rechtshemisphärische Aktivierung während dieses Tests.

Die anatomischen Aktivitätsschwerpunkte der Probanden, die in der rechten Hemisphäre signifikante Aktivität im Broca-Areal zeigten, lagen bei zehn Probanden im Gyrus temporalis superior und bei den restlichen drei Probanden im Gyrus temporalis medialis.

Bezogen auf die Brodmann-Areale ergab sich, dass die Aktivitätsschwerpunkte am häufigsten im BA 22 lagen. Hier zeigten sechs der Probanden signifikante Aktivität. Bei den übrigen Probanden dieses Kollektivs lagen die Aktivitätsschwerpunkte bei vier Probanden im BA 21, bei zwei Probanden im BA 41 und bei einem Probanden im BA 42 (Tabelle 9).

\subsubsection{WERNICKE-REgION LINKS IM KOLLEKTIV E}

Bei der Auswertung des Sprachtests „Wortgenerierung“ konnte für alle zwölf Patienten mit fokaler Epilepsie in den definierten ROls im Gebiet der Wernicke-Region der linken Hemisphäre statistisch signifikante kortikale Aktivierung nachgewiesen werden. 
Bei sechs Patienten lagen die anatomischen Aktivitätsschwerpunkte im Gyrus temporalis medialis, bei fünf Patienten im Gyrus temporalis superior und bei einem Patienten subgyral.

Bezogen auf die Brodmann-Areale lagen bei sechs Patienten die Aktivitätsschwerpunkte im BA 22. Dieses Areal war damit am häufigsten vertreten. Dem Aktivitätsschwerpunkt des Patienten 34 im Gyrus temporalis medialis konnte kein BA zugeordnet werden. Die restlichen fünf Patienten zeigten dagegen uneinheitliche Aktivitätsschwerpunkte verteilt auf die BA 13, 20, 37, 41 und 42 (Tabelle 10).

\subsubsection{WeRnicke-REgION RECHTS IM KolLEKTIV E}

Neun der zwölf Patienten mit fokaler Epilepsie zeigten statistisch signifikante rechtshemisphärische kortikale Aktivierung während des Sprachtests "Wortgenerierung" in den definierten ROls im Gebiet der Wernicke-Region. Für drei Patienten konnte keine Aktivierung in der rechten Hemisphäre ermittelt werden.

Die anatomischen Aktivitätsschwerpunkte der Patienten mit Aktivierung in der rechten Hemisphäre lagen bei sieben Patienten im Gyrus temporalis superior und bei den anderen beiden Patienten im Gyrus temporalis medialis.

Bezogen auf die Brodmann-Areale lagen die Aktivitätsschwerpunkte von fünf Patienten im BA 22. Bei drei Patienten lagen die Schwerpunkte der Aktivierung im BA 41 und bei einem Patienten im BA 13 (Tabelle 11).

\subsubsection{VERGLEICH DER LIS IN DER WERNICKE-REgION WÄHREND DES SPRACHTESTS „WORTGENERIERUNG“}

Für die funktionelle Aktivierung innerhalb der Wernicke-Region während des Sprachtests "Wortgenerierung" wurde mit Hilfe der Anzahl der signifikant aktivierten Bildpunkte einer ROI in der linken und der zugehörigen ROI in der rechten Hemisphäre für jeden Probanden der LI berechnet.

Anschließend wurde die Verteilung der LIs in der Gruppe der Patienten mit fokaler Epilepsie und im Kontrollkollektiv miteinander verglichen. Im Kontrollkollektiv konnte in den definierten ROls in der Wernicke-Region während des Sprachtests „Wortgenerierung“ für alle 23 Probanden ein LI berechnet werden. 16 Probanden (70\%) zeigten eine Lateralisierung nach links, sechs Probanden (26\%) eine Lateralisierung nach rechts und ein Proband (4\%) wies eine symmetrische Lateralisierung auf.

In der Gruppe der Patienten mit fokaler Epilepsie konnte ebenfalls für jeden der zwölf Patienten ein LI berechnet werden. Neun Patienten (75\%) dieses Kollektivs zeigten eine Lateralisierung nach links, die übrigen drei Patienten $(25 \%)$ zeigten eine rechtsgerichtete Lateralisierung (Tabelle 12). 
Der Vergleich der LIs beider Gruppen ergab, dass die linksgerichteten LIs der Kontrollgruppe einen Wertebereich von 0,194 bis 1 umfassten mit einem Median von 1. Dieser Median lag über dem der Patienten mit fokaler Epilepsie, deren linksgerichtete LIs einen Wertebereich von 0,117 bis 1 mit einem Median von 0,648 umfassten.

Die rechtsgerichteten LIs der Kontrollgruppe umfassten einen Wertebereich von -1 bis -0,103 mit einem Median von -0,336. Im Vergleich dazu umfassten die rechtsgerichteten LIs der Patienten mit fokaler Epilepsie Werte von -0,423 bis $-0,25$ mit einem Median von -0,266, der damit über dem Median der Normalprobanden lag.

In der Kontrollgruppe zeigte ein Proband eine symmetrische Aktivierung mit einem LI von -0,007. In der Gruppe der Patienten mit fokaler Epilepsie hingegen wiesen alle Patienten eine Lateralisierung auf (Tabelle 24).

Die Verteilung der LIs der fMRT-Daten zwischen der Gruppe der Kontrollprobanden und der Gruppe der Patienten mit fokaler Epilepsie wurde mit Hilfe des Mann-WhitneyU-Tests untersucht. $\mathrm{Zu}$ einem Fehlerniveau von $5 \%$ bestand kein Unterschied hinsichtlich der funktionellen Lateralisierung zwischen der Gruppe der Patienten mit fokaler Epilepsie und der Gruppe der Kontrollprobanden im Bereich des WernickeAreals während des Sprachtests „Wortgenerierung“ ( $p=0,605)$ (Tabelle 27). Auch der Vergleich des Kontrollkollektivs mit den Patienten, die eine ausschließlich linkshemisphärische Epileptogenese aufwiesen, zeigte keinen signifikanten Unterschied hinsichtlich der funktionellen Lateralisierung zwischen den Gruppen $(p=0,801)$ (Tabelle 28).

\subsubsection{SpRachtest „SATZgenerierung“ IN DER BROCA-Region}

22 der 23 Probanden des Kontrollkollektivs zeigten während des Sprachtests "Satzgenerierung" eine signifikante Aktivierung im Bereich der Broca-Region in mindestens einer der beiden Hemisphären. Bei Proband 18 (Tabelle 13 und 14) des Kontrollkollektivs konnte weder in der linken noch in der rechten Hemisphäre signifikante Aktivierung während dieses Abschnitts des Experiments nachgewiesen werden. Bereits bei der Auswertung der Aktivierung während des Sprachtests „Wortgenerierung“ fiel auf, dass dieser Proband in der Broca-Region keine Aktivierung zeigte (Tabelle 3 und 4).

Bei der Auswertung der Gruppe der Patienten mit fokaler Epilepsie konnte für alle zwölf Patienten in den definierten ROls im Gebiet der Broca-Region während des Sprachtests "Satzgenerierung“ eine statistisch signifikante kortikale Aktivierung in beiden Hemisphären ermittelt werden (Tabelle 13 bis 16).

\subsubsection{BROCA-REgION LINKS IM KOLLEKTIV N}

Für 22 der 23 Probanden des Kontrollkollektivs konnte in den definierten ROls im Gebiet der Broca-Region der linken Hemisphäre statistisch signifikante kortikale 
Aktivierung im Zusammenhang mit dem Sprachtest „Satzgenerierung“ nachgewiesen werden.

Die anatomischen Aktivitätsschwerpunkte lagen bei 18 Probanden im Gyrus frontalis inferior, bei zwei Probanden in der Inselrinde und bei jeweils einem Probanden im Gyrus frontalis medialis und im Gyrus praecentralis.

In Bezug auf die Brodmann-Areale lagen die Aktivitätsschwerpunkte bei 11 Probanden und damit am häufigsten im BA 45. Bei fünf Probanden lagen die Aktivitätsschwerpunkte im BA 13, bei jeweils zwei Probanden im BA 44 und 46 und schließlich bei jeweils einem Probanden im BA 9 und 47 (Tabelle 13).

\subsubsection{BROCA-REgION RECHTS IM KOLLEKTIV N}

Bei acht der 23 Probanden und damit bei weniger als der Hälfte des Kontrollkollektivs konnte in den definierten ROls im Gebiet der Broca-Region der rechten Hemisphäre während des Sprachtests "Satzgenerierung" eine statistisch signifikante kortikale Aktivierung beobachtet werden. 15 Probanden zeigten keine rechtshemisphärische Aktivierung bei diesem Test.

Die anatomischen Aktivitätsschwerpunkte der Probanden, die in der rechten Hemisphäre signifikante Aktivität im Broca-Areal zeigten, lagen bei drei Probanden im Gyrus frontalis inferior, bei weiteren drei Probanden im Gyrus frontalis medialis und bei zwei Probanden in der Inselrinde.

In Bezug auf die Brodmann-Areale konnten den Aktivitätsschwerpunkten von jeweils zwei Probanden die BA 9, 13 und 46 zugeordnet werden. Bei einem Probanden lag der Aktivitätsschwerpunkt im BA 45. Der Aktivierung eines weiteren Probanden mit Schwerpunkt im Gyrus frontalis inferior konnte kein BA zugeordnet werden (Tabelle 14).

\subsubsection{BROCA-REGION LINKS IM KOLLEKTIV E}

Bei der Auswertung des Sprachtests „Satzgenerierung“ zeigten alle zwölf Patienten mit fokaler Epilepsie in den definierten ROls im Gebiet der Broca-Region der linken Hemisphäre statistisch signifikante kortikale Aktivierung.

Bei neun Patienten lagen die anatomischen Aktivitätsschwerpunkte im Gyrus frontalis inferior, bei zwei Patienten in der Insel und bei einem Patienten im Gyrus praecentralis.

Bezogen auf die Brodmann-Areale konnten den Aktivitätsschwerpunkten von fünf Patienten das BA 13 zugeordnet werden, bei vier Patienten lagen die Aktivitätsschwerpunkte im BA 45, bei zwei Patienten im BA 44 und bei einem Patienten im BA 9 (Tabelle 15). 


\subsubsection{BROCA-REGION RECHTS IM KOLLEKTIV E}

Die Auswertung der kortikalen Aktivierung in der rechten Hemisphäre während des Sprachtests „Satzgenerierung“ zeigte ebenfalls bei allen zwölf Patienten mit fokaler Epilepsie in den definierten ROIs im Gebiet der Broca-Region statistisch signifikante Aktivierung.

Die anatomischen Aktivitätsschwerpunkte der Patienten lagen bei fünf Patienten im Gyrus frontalis inferior, bei vier Patienten in der Inselrinde und bei drei Patienten im Gyrus frontalis medialis.

Bezogen auf die Brodmann-Areale ergab sich somit, dass bei jeweils vier Patienten mit kortikaler Aktivierung in der rechten Hemisphäre den Aktivitätsschwerpunkten die BA 13 und 46 zugeordnet werden konnten. Bei zwei Patienten lagen die Aktivitätsschwerpunkte im BA 45 und bei jeweils einem Patienten im BA 9 und BA 44 (Tabelle 16).

\subsubsection{VERGLEICH DER LIS IN DER BROCA-REGION WÄHREND DES SPRACHTESTS "SATZGENERIERUNG“}

Anhand der Anzahl signifikant aktivierter Bildpunkte einer ROI in der linken und der zugehörigen ROI der rechten Hemisphäre wurde für jeden Probanden der LI für die funktionelle Aktivierung innerhalb der Broca-Region während des Sprachtests „Satzgenerierung“ berechnet.

Die LIs der Gruppe der Patienten mit fokaler Epilepsie wurden anschließend mit denen des Kontrollkollektivs verglichen. Hinsichtlich der Aktivierung in den definierten ROls in der Broca-Region während des Sprachtests „Satzgenerierung“ konnte für 22 der 23 Probanden des Normalkollektivs ein LI berechnet werden. Ein Proband zeigte während des gesamten Tests weder in der linken noch in der rechten Hemisphäre Aktivierung. Von den übrigen 22 Probanden zeigten 21 (95\%) eine linksgerichtete Lateralisierung und ein Proband (5\%) zeigte eine rechtsgerichtete Lateralisierung.

In der Gruppe der Patienten mit fokaler Epilepsie konnte dagegen für jeden der zwölf Patienten ein LI berechnet werden. Acht Patienten (67\%) dieses Kollektivs zeigten eine Lateralisierung nach links, zwei Patienten (17\%) zeigten eine Lateralisierung nach rechts und weitere zwei Patienten (17\%) zeigten eine symmetrische Aktivierung (Tabelle 17).

Im Vergleich der LIs beider Gruppen umfassten die linksgerichteten LIs der Kontrollgruppe einen Wertebereich von 0,24 bis 1 mit einem Median von 1. Dieser Median lag über dem der Patienten mit fokaler Epilepsie, deren linksgerichteten Lls einen Wertebereich von 0,263 bis 0,69 mit einem Median von 0,52 umfassten.

Der Proband der Kontrollgruppe mit rechtsgerichteter Lateralisierung wies einen LI von $-0,795$ auf. Die rechtsgerichteten LIs der Patienten mit fokaler Epilepsie umfassten die Werte $-0,534$ und $-0,399$ mit einem Median von $-0,466$. 
In der Kontrollgruppe wiesen alle Probanden eine Lateralisierung auf. Die LIs der symmetrischen Aktivierung in der Gruppe der Patienten mit fokaler Epilepsie umfassten die Werte -0,059 und 0,059 mit einem Median von 0,0001 (Tabelle 25).

Mit Hilfe des Mann-Whitney-U-Tests wurden die funktionellen LIs beider Gruppen hinsichtlich ihrer Verteilung untersucht. Im Bereich des Broca-Areals unterschied sich die funktionelle Lateralisierung des Patientenkollektivs signifikant $(p=0,0002)$ von der Lateralisierung des Kontrollkollektivs während des Sprachtests „Satzgenerierung“ (Tabelle 27). Diese Signifikanz blieb auch bei dem Vergleich der funktionellen Lateralisierung des Kontrollkollektivs mit der Lateralisierung in dem Kollektiv der Patienten mit ausschließlich linkshemisphärischer Epileptogenese bestehen $(p=0,001)$ (Tabelle 28).

\subsubsection{Sprachtest „SATZgenerierung“ iN DER WeRniCKe-Region}

Alle 23 Probanden des Kontrollkollektivs zeigten während des Sprachtests "Satzgenerierung“ signifikante Aktivierung im Bereich der Wernicke-Region in mindestens einer der beiden Hemisphären.

Auch bei der Auswertung des Kollektivs der Patienten mit fokaler Epilepsie zeigten alle zwölf Patienten in den definierten ROIs im Gebiet der Wernicke-Region während des Sprachtests "Satzgenerierung“ statistisch signifikante kortikale Aktivierung in mindestens einer der beiden Hemisphären (Tabelle 18 bis 21).

\subsubsection{WERNICKE-REgION LINKS IM KOLLEKTIV N}

In den definierten ROIs im Gebiet der Wernicke-Region der linken Hemisphäre konnte bei 22 der 23 Probanden des Kontrollkollektivs statistisch signifikante kortikale Aktivierung im Zusammenhang mit dem Sprachtest „Satzgenerierung“ ermittelt werden. Nur Proband 8 zeigt keine Aktivierung in der linken Hemisphäre während dieses Tests.

Die anatomischen Aktivitätsschwerpunkte der Probanden lagen bei zwölf Probanden im Gyrus temporalis superior und bei neun Probanden im Gyrus temporalis medialis. Lediglich ein Proband zeigte Aktivierung in der Inselrinde.

Bezogen auf die Brodann-Areale lagen bei 14 Probanden und damit am häufigsten die Aktivitätsschwerpunkte im BA 22. Bei vier Probanden lagen die Aktivitätsschwerpunkte im BA 21, bei drei Probanden im BA 42 und bei einem Probanden im BA 13 (Tabelle 18).

\subsubsection{WERNICKE-REgION RECHTS IM KOLLEKTIV N}

Bei der Auswertung der definierten ROls im Gebiet der Wernicke-Region der rechten Hemisphäre zeigten dagegen nur 13 der 23 Probanden des Kontrollkollektivs statistisch signifikante kortikale Aktivierung im Zusammenhang mit dem Sprachtest "Satzgenerierung“. 
Die anatomischen Aktivitätsschwerpunkte der Probanden, die in der rechten Hemisphäre signifikante Aktivität im Broca-Areal zeigten, lagen bei zwölf der 13 Probanden im Gyrus temporalis superior und bei einem Probanden im Gyrus temporalis medialis.

Bezogen auf die Brodmann-Areale konnte den Koordinaten der Aktivitätsschwerpunkte am häufigsten das BA 22 zugeordnet werden. Hier zeigten zehn der Probanden signifikante Aktivität. Bei jeweils einem weiteren Probanden lagen die Aktivitätsschwerpunkte im BA 21, 41 und 42 (Tabelle 19).

\subsubsection{WERNICKE-REgION LINKS IM KolLEKTIV E}

Die Auswertung des Kollektivs der Patienten mit fokaler Epilepsie zeigte bei elf von zwölf Patienten statistisch signifikante kortikale Aktivierung in den definierten ROls im Gebiet der Wernicke-Region der linken Hemisphäre während des Sprachtests "Satzgenerierung“.

Bei sechs Patienten lagen die anatomischen Aktivitätsschwerpunkte im Gyrus temporalis superior, bei drei Patienten im Gyrus temporalis medialis und bei einem Patienten subgyral.

Bezogen auf die Brodmann-Areale lagen bei jeweils vier Patienten die Aktivitätsschwerpunkte im BA 22 und BA 41. Die Aktivitätsschwerpunkte der anderen Patienten lagen jeweils einmal in dem BA 13 und BA 37. Lediglich die Koordinaten des linkshemisphärischen Aktivitätsschwerpunktes des Patienten 34 lagen nicht in der grauen Substanz. Ihnen konnte deshalb kein BA zugeordnet werden (Tabelle 20).

\subsubsection{WeRnicke-Region RECHTS IM KolLeKtiv E}

Neun der zwölf Patienten mit fokaler Epilepsie zeigten bei der Auswertung der definierten ROls im Gebiet der Wernicke-Region statistisch signifikante Aktivierung in der rechten Hemisphäre während des Sprachtests „Satzgenerierung“.

Die anatomischen Aktivitätsschwerpunkte lagen bei allen Patienten im Temporallappen. Bei sechs Patienten lag die Aktivierung im Gyrus temporalis superior, bei den übrigen drei Patienten im Gyrus temporalis medialis.

Nur bei acht der neun Patienten, die kortikale Aktivierung in der rechten Hemisphäre zeigten, konnten den Aktivitätsschwerpunkten Brodmann-Areale zugeordnet werden. Bei vier Patienten lagen die Aktivitätsschwerpunkte im BA 22, bei drei Patienten im BA 41 und bei einem Patienten im BA 21. Dem Aktivitätsschwerpunkt im Gyrus temporalis medialis des Patienten 25 konnte kein BA zugeordnet werden (Tabelle 21). 


\subsubsection{VERGLEICH DER LIS IN DER WERNICKE-REGION WÄHREND DES SPRACHTESTS „SATZGENERIERUNG“}

Auch für die funktionelle Aktivierung innerhalb der Wernicke-Region während des Sprachtests „Satzgenerierung“ wurde für jeden Probanden der LI auf Grundlage der Anzahl der signifikant aktivierten Voxel der linkshemisphärischen ROls und der zugehörigen rechtshemisphärischen ROls berechnet.

Im Vergleich der LIs der Gruppe der Patienten mit fokaler Epilepsie mit denen des Kontrollkollektivs konnte für alle 23 Probanden des Kontrollkollektivs ein Lateralitätsindex berechnet werden. 18 Probanden (78\%) zeigten eine Lateralisierung nach links, drei Probanden (13\%) eine Lateralisierung nach rechts und zwei Probanden (9\%) zeigten eine symmetrische Aktivierung.

In der Gruppe der Patienten mit fokaler Epilepsie konnte ebenfalls für jeden der zwölf Patienten ein LI berechnet werden. Sechs Patienten (50\%) zeigten eine Lateralisierung nach links, vier Patienten (33\%) eine Lateralisierung nach rechts und zwei Patienten (17\%) zeigten eine symmetrische Aktivierung (Tabelle 22).

Im Vergleich der LIs beider Gruppen umfassten die linksgerichteten LIs der Kontrollgruppe einen Wertebereich von 0,105 bis 1 mit einem Median von 1. Dieser Median lag über dem der Patienten mit fokaler Epilepsie, deren linksgerichteten LIs einen Wertebereich von 0,119 bis 1 mit einem Median von 0,893 umfassten.

Die rechtsgerichteten LIs der Kontrollgruppe umfassten einen Wertebereich von -1 bis $-0,202$ mit einem Median von -0,516. Im Vergleich dazu umfassten die rechtsgerichteten LIs der Patienten mit fokaler Epilepsie Werte von -1 bis $-0,26$ mit einem Median von -0,346, der damit über dem Median der Normalprobanden lag.

In der Kontrollgruppe umfassten die LIs der Probanden mit symmetrischer Aktivierung Werte von $-0,066$ bis 0,0003 mit einem Median von -0,033. Die LIs der symmetrischen Aktivierung in der Gruppe der Patienten mit fokaler Epilepsie umfassten Werte von $-0,099$ bis 0,069 mit einem Median von -0,015 (Tabelle 26).

Mit Hilfe des Mann-Whitney-U-Tests wurden die funktionellen LIs beider Gruppen hinsichtlich ihrer Verteilung innerhalb der Gruppe der Kontrollprobanden und der Gruppe der Patienten mit fokaler Epilepsie untersucht. Die funktionelle Lateralisierung des Patientenkollektivs unterschied sich bei einem Fehlerniveau von $5 \%$ nicht signifikant $(p=0,164)$ von der des Kontrollkollektivs im Bereich des Wernicke-Areals während des Sprachtests „Satzgenerierung“ (Tabelle 27). Auch der Vergleich des Patientenkollektivs mit der Gruppe der Patienten mit ausschließlich linkshemisphärischer Epileptogenese zeigte keinen signifikanten Unterschied hinsichtlich der Verteilung der funktionellen Lateralisierung innerhalb der Gruppen $(p=0,346)$ (Tabelle 28). 


\subsection{DTI-Ergebnisse}

Die FA-Karten der diffusionsgewichteten Daten wurden mit dem anatomischen dreidimensionalen Datensatz verknüpft. Anschließend konnten die ROls, die bereits im Rahmen der funktionellen Datenauswertung definiert wurden („Satz Broca“, „Wort Broca“, „Satz Wernicke“, „Wort Wernicke“), auf die FA-Karten übertragen werden. Die funktionellen ROls dienten als Ausgangspunkt für die Bestimmung der strukturellen ROls, die analog zu den zugehörigen funktionellen ROls benannt wurden. Beide Gruppen wurden auf eine Asymmetrie in der FA der weißen Substanz hin untersucht und miteinander verglichen.

Die Auswertung der DTI-Daten beider definierten strukturellen ROIs im Broca-Areal zeigte, dass sich die Verteilung der Als innerhalb der untersuchten Gruppen signifikant voneinander unterschied. Dieses galt auch für den Vergleich des Kontrollkollektivs mit den Patienten mit ausschließlich linkshemisphärischer Epileptogenese. Die Nullhypothese konnte in diesen Regionen abgelehnt werden.

Für das Wernicke-Areal hingegen konnte die Auswertung der beiden definierten strukturellen ROls keinen signifikanten Unterschied in der Verteilung der Als innerhalb der Gruppen nachweisen. Auch der Vergleich des Kontrollkollektivs mit den Patienten mit ausschließlich linkshemisphärischer Epileptogenese ergab keinen signifikanten Unterschied in der Verteilung der Als innerhalb der Gruppen. Die Nullhypothese durfte in der Wernicke-Region somit nicht abgelehnt werden.

\subsubsection{DTI-AusWERTUNG DER BROCA-REgION}

In der Broca-Region beider Kollektive wurde die ROI „Satz Broca“, die im Rahmen der funktionellen Datenauswertung des Sprachtests „Satzgenerierung“ bereits definiert wurde, sowie die ROI „Wort Broca“, die im Rahmen der funktionellen Datenauswertung des Sprachtests „Wortgenerierung“ zuvor definiert wurde, auf die FA-Karten angewendet. Diese dienten anschließend als Ausgangspunkte für die Definition der strukturellen ROls.

\subsubsection{ASYMMETRIEINDIZES DER STRUKTURELLEN ROI „WORT BROCA“}

In der Broca-Region bestand bei 19 der 23 Probanden des Normalkollektivs eine Asymmetrie der FA. 17 Probanden (74\%) zeigten eine Asymmetrie mit höheren FAWerten auf der linken Seite und zwei Probanden (9\%) zeigten höhere FA-Werte auf der rechten Seite. Die übrigen vier Normalprobanden (17\%) zeigten eine symmetrische Verteilung der FA.

Neun der zwölf Patienten mit fokaler Epilepsie zeigten bei der Auswertung dieser strukturellen ROI in der Broca-Region eine Asymmetrie der FA. Sechs Patienten (50\%) zeigten eine Asymmetrie mit linkshemisphärisch höherer FA und drei (25\%) eine 
Asymmetrie mit rechtshemisphärisch höherer FA. Die übrigen drei Patienten (25\%) wiesen eine symmetrische Verteilung der FA auf (Tabelle 29).

\subsubsection{Vergleich der Als In DER StRUkturellen ROI „WORT BROCA“}

Die Als der strukturellen ROI „Wort Broca“ beider Gruppen wurden miteinander verglichen.

Im Vergleich der strukturellen Asymmetrie beider Gruppen umfassten die Als mit linksgerichtet höherer FA der Kontrollgruppe einen Wertebereich von 0,118 bis 1 mit einem Median von 1. Dieser Median lag über dem der Patienten mit fokaler Epilepsie, deren linksgerichtete Als einen Wertebereich von 0,14 bis 1 mit einem Median von 0,23 umfassten.

Die Als mit rechtsgerichtet höherer FA der Kontrollgruppe umfassten einen Wertebereich von $-0,29$ bis $-0,215$ mit einem Median von -0,252. Im Vergleich dazu umfassten die rechtsgerichteten Als der Patienten mit fokaler Epilepsie Werte von $-0,109$ bis $-0,516$ mit einem Median von $-0,164$.

In der Kontrollgruppe umfassten die Als der Probanden mit symmetrischer Verteilung der FA Werte zwischen -0,063 und 0,092 mit einem Median von 0,0695. Die Als mit symmetrischer Verteilung in der Gruppe der Patienten mit fokaler Epilepsie umfassten Werte von $-0,052$ bis 0,095 mit einem Median von 0,015 (Tabelle 33).

Mit Hilfe des Mann-Whitney-U-Tests wurden die Als beider Gruppen hinsichtlich ihrer Verteilung innerhalb der Gruppe der Kontrollprobanden und der Gruppe der Patienten mit fokaler Epilepsie untersucht. Die statistische Auswertung ergab, dass sich die strukturelle Asymmetrie der FA des Patientenkollektivs von der des Kontrollkollektivs im Bereich des Broca-Areals für die strukturellen ROls „Wort Broca“ signifikant unterschied $(p=0,017)$ (Tabelle 37). Bei dem Vergleich der Kontrollprobanden mit den Patienten mit ausschließlich linkshemisphärischer Epileptogenese zeigte sich ebenfalls eine signifikant unterschiedliche strukturelle Asymmetrie der FA $(p=0,012)$ (Tabelle 38).

\subsubsection{ASYMMETRIEINDIZES DER STRUKTURELLEN ROI „SATZ BROCA“}

Bei der Auswertung der strukturellen ROIs, die auf Grundlage der funktionellen Datenauswertung des Sprachtests "Satzgenerierung" definiert wurden, konnte bei 21 der 23 Probanden des Normalkollektivs in der Broca-Region eine Asymmetrie der FA nachgewiesen werden. Bei 19 Probanden (83\%) zeigte sich eine Asymmetrie mit höheren FA-Werten in der linken Hemisphäre und bei zwei Probanden (9\%) mit höheren FA-Werten in der rechten Hemisphäre. Die übrigen beiden Normalprobanden (9\%) zeigten eine symmetrische Verteilung der FA.

In der Gruppe der Patienten mit fokaler Epilepsie wurde bei dieser Auswertung eine Asymmetrie der FA bei sechs von zwölf Patienten beobachtet. Drei Patienten (25\%) zeigten eine Asymmetrie mit linkshemisphärisch höherer FA und drei (25\%) eine 
Asymmetrie mit rechtshemisphärisch höherer FA. Die übrigen sechs Patienten (50\%) mit fokaler Epilepsie wiesen eine symmetrische Verteilung der FA auf (Tabelle 30).

\subsubsection{VERGLEICH DER AIS IN DER STRUKTURELLEN ROI „SATZ BROCA“}

Auch für die strukturelle ROI „Satz Broca“ wurden die Als beider Gruppen miteinander verglichen.

Die Als mit linksgerichtet höherer FA der Kontrollgruppe umfassten einen Wertebereich von 0,286 bis 1 mit einem Median von 1. Dieser Median lag über dem der Patienten mit fokaler Epilepsie, deren linksgerichtete Als einen Wertebereich von 0,112 bis 0,426 mit einem Median von 0,257 umfassten.

Die Als mit rechtsgerichtet höherer FA der Kontrollgruppe umfassten einen Wertebereich von -1 bis -0,206 mit einem Median von -0,603. Im Vergleich dazu umfassten die rechtsgerichteten Als der Patienten mit fokaler Epilepsie Werte von $-0,374$ bis $-0,112$ mit einem Median von $-0,279$.

In der Kontrollgruppe umfassten die Als der Probanden mit symmetrischer Verteilung der FA Werte zwischen 0,016 und 0,071 mit einem Median von 0,044. Die Als mit symmetrischer Verteilung in der Gruppe der Patienten mit fokaler Epilepsie umfassten Werte von $-0,045$ bis 0,084 mit einem Median von -0,005 (Tabelle 34).

Ebenso wurde die Verteilung der Als beider Gruppen mit Hilfe des Mann-Whitney-UTests untersucht. Die statistische Auswertung zeigte einen signifikanten Unterschied $(p=0,0002)$ der strukturellen Asymmetrie der FA des Patientenkollektivs und des Kontrollkollektivs im Bereich der strukturellen ROI „Satz Broca“ (Tabelle 37). Auch für den Vergleich des Kontrollkollektivs mit den Patienten mit ausschließlich linkshemisphärischer Epileptogenese zeigte sich ein signifikanter Unterschied $(p=0,0017)$ der strukturellen Asymmetrie der FA in der untersuchten ROI (Tabelle 38).

\subsubsection{ASYMMETRIEINDIZES IN DER WERNICKE-REGION}

Analog zur Auswertung der FA der Broca-Region wurden in der Wernicke-Region beider Kollektive die ROls "Satz Wernicke“ und „Wort Wernicke“ auf die FA-Karten angewendet als Ausgangspunkt für die Definition der strukturellen ROls.

\subsubsection{ASYMMETRIEINDIZES DER STRUKTURELLEN ROI „WORT WERNICKE“}

Bei der Auswertung der strukturellen ROls, die auf Grundlage der funktionellen Datenauswertung des Sprachtests „Wortgenerierung“ bestimmt wurden, ließ sich im Kollektiv der Normalprobanden bei 19 der 23 Probanden eine Asymmetrie der FA in der Wernicke-Region beobachten. 13 Probanden (57\%) zeigten eine Asymmetrie mit höherer FA auf der linken Seite und sechs Probanden (26\%) zeigten eine Asymmetrie mit höherer FA auf der rechten Seite. Die übrigen vier Normalprobanden (17\%) zeigten eine symmetrische Verteilung der FA. 
In der Gruppe der Patienten mit fokaler Epilepsie wurde bei der Auswertung dieser ROls bei sieben der zwölf Patienten eine Asymmetrie beobachtet. Vier Patienten (48\%) zeigten eine Asymmetrie mit linkshemisphärisch höheren FA-Werten und drei $(25 \%)$ Asymmetrie mit rechtshemisphärisch höherer FA. Die übrigen fünf Patienten (42\%) mit fokaler Epilepsie wiesen eine symmetrisch verteilte FA auf (Tabelle 31).

\subsubsection{VeRGLEICH DER AIS IN DER STRUKTURELLEN ROI „WORT WERNICKE“}

Der Vergleich der Als für die strukturellen ROls "Wort Wernicke“ beider Gruppen ergab, dass die Als mit linksgerichtet höherer FA der Kontrollgruppe einen Wertebereich von 0,193 bis 1 mit einem Median von 1 umfassten. Dieser Median lag über dem der Patienten mit fokaler Epilepsie, deren linksgerichtete Als einen Wertebereich von 0,122 bis 1 umfassten mit einem Median von 1.

Die Als mit rechtsgerichtet höheren FA-Werten der Kontrollgruppe umfassten einen Wertebereich von -1 bis $-0,179$ mit einem Median von -0,356. Im Vergleich dazu umfassten die rechtsgerichteten Als der Patienten mit fokaler Epilepsie Werte von $-0,481$ bis $-0,125$ mit einem Median von $-0,261$.

In der Kontrollgruppe umfassten die Als der Probanden mit symmetrischer Verteilung der FA Werte zwischen $-0,021$ und 0,029 mit einem Median von 0,017. Die Als mit symmetrischer Verteilung in der Gruppe der Patienten mit fokaler Epilepsie umfassten Werte von $-0,085$ bis 0,053 mit einem Median von 0,002 (Tabelle 35).

Die Als beider Gruppen wurden mit Hilfe des Mann-Whitney-U-Tests hinsichtlich ihrer Verteilung innerhalb der Gruppe der Kontrollprobanden und der Gruppe der Patienten mit fokaler Epilepsie untersucht. Die statistische Auswertung ergab keinen signifikanten Unterschied $(p=0,332)$ der strukturellen Asymmetrie der FA zwischen dem Patientenkollektiv und dem Kontrollkollektiv in der strukturellen ROI „Wort Wernicke“ (Tabelle 37). Auch der Vergleich der Gruppe der Patienten mit ausschließlich linkshemisphärischer Epileptogenese mit dem Kontrollkollektiv konnte für diese strukturelle ROI keinen signifikanten Unterschied $(p=0,753)$ der strukturellen Asymmetrie der FA zwischen den Gruppen nachweisen (Tabelle 38).

\subsubsection{AsYMmEtRIEINDIZES DER STRUKTURELLEN ROI „SATZ WERNICKE“}

Bei der Auswertung der FA in den ROIs, die auf Grundlage der funktionellen Datenauswertung des Sprachtests "Satzgenerierung“ in der Wernicke-Region definiert wurden, zeigten 17 der 23 Normalprobanden eine Asymmetrie. Die Asymmetrie der FA ergab bei 14 Probanden (61\%) auf der linken Seite höhere FA-Werte und bei drei Probanden (13\%) auf der rechten Seite höhere FA-Werte. Die übrigen sechs Normalprobanden (26\%) zeigten eine symmetrische Verteilung der FA.

Die Auswertung der FA in der Gruppe der Patienten mit fokaler Epilepsie ergab für diese strukturelle ROI bei sieben der 12 Patienten eine Asymmetrie. Fünf Patienten (42\%) zeigten eine Asymmetrie mit linkshemisphärisch höherer FA und zwei $(17 \%)$ 
eine Asymmetrie mit rechtshemisphärisch höherer FA. Die übrigen fünf Patienten (42\%) mit fokaler Epilepsie wiesen eine symmetrisch verteilte FA auf (Tabelle 32).

\subsubsection{Vergleich der Als In DeR StRUktURELlen ROI „SATZ WeRnickE“}

Auch die Als der Wernicke-Regionen beider Gruppen wurden miteinander verglichen und es zeigte sich, dass die Als mit linksgerichtet höherer FA der Kontrollgruppe einen Wertebereich von 0,156 bis 1 mit einem Median von 1 umfassten. Dieser entsprach dem der Patienten mit fokaler Epilepsie, deren linksgerichteten Als einen Wertebereich von 0,166 bis 1 umfassten mit ebenfalls einem Median von 1.

Die Als mit rechtsgerichtet höherer FA der Kontrollgruppe umfassten einen Wertebereich von -0,165 bis -1 mit einem Median von -0,82. Im Vergleich dazu umfassten die rechtsgerichteten Als der Patienten mit fokaler Epilepsie Werte von $-0,249$ bis -1 mit einem Median von $-0,624$.

In der Kontrollgruppe umfassten die Als der Probanden mit symmetrischer Verteilung der FA Werte zwischen -0,036 und 0,085 mit einem Median von 0,036. Die symmetrisch verteilten Als in der Gruppe der Patienten mit fokaler Epilepsie umfassten Werte von -0,066 und 0,058 mit einem Median von -0,007 (Tabelle 36).

Die Verteilung der Als innerhalb der beiden Gruppen wurde mit dem Mann-Whitney-UTest untersucht. Die statistische Auswertung ergab, dass sich die strukturelle Asymmetrie der FA des Patientenkollektivs von der des Kontrollkollektivs für die strukturelle ROI „Satz Wernicke“ nicht signifikant unterschied $(p=0,175)$ (Tabelle 37). Auch die strukturelle Asymmetrie der FA der Patienten mit ausschließlich linkshemisphärischer Epileptogenese unterschied sich nicht signifikant $(p=0,304)$ von der des Kontrollkollektivs (Tabelle 38).

\subsection{Kombinierte Analyse von fMRT und DTI}

Schließlich wurden die Zusammenhänge zwischen funktioneller Lateralisierung und struktureller Asymmetrie untersucht. Es wurde überprüft, ob die berechneten Indizes beider Messungen gleichsinnige Aussagen hinsichtlich der Lateralität machten.

Mit Hilfe des Vorzeichentests wurden die LIs der funktionellen und die Als der diffusionsgewichteten MRT-Daten der zugehörigen ROls miteinander verglichen. Dieser Vergleich wurde für alle Studiengruppen getrennt voneinander durchgeführt.

In der Kontrollgruppe konnte zu einem Fehlerniveau von 5\% kein signifikanter Unterschied hinsichtlich der Lateralisierung der funktionellen Daten und der strukturellen Asymmetrie gezeigt werden. Dieses galt sowohl für die Daten der Broca$(p=0,077)$ und der Wernicke-Region $(p=0,773)$, die während des Sprachtests „Satzgenerierung“ erhoben wurden, als auch für die Daten der Broca- $(p=0,096)$ und 
der Wernicke-Region $(p=0,546)$, die während des Sprachtests „Wortgenerierung“ ermittelt wurden (Tabelle 39).

In der Gruppe der Patienten mit fokaler Epilepsie konnte die Auswertung des Sprachtests "Satzgenerierung“ zu einem Fehlerniveau von 5\% keinen signifikanten Unterschied hinsichtlich der Lateralisierung der funktionellen Daten und der strukturellen Asymmetrie zeigen. Weder in der Broca-Region $(p=0,149)$ noch in der Wernicke-Region $(p=0,724)$ durfte die Nullhypothese verworfen werden.

Die Auswertung des Sprachtests "Wortgenerierung“ in dem Patientenkollektiv zeigte in der Broca-Region ebenfalls zu einem Fehlerniveau von 5\% keinen signifikanten Unterschied der funktionellen Lateralisierung und der strukturellen Asymmetrie $(p=0,228)$. Anders verhielt es sich in der Wernicke-Region. Hier zeigte sich im Kollektiv der Patienten mit fokaler Epilepsie während des Sprachtests „Wortgenerierung“ ein signifikanter Unterschied zwischen der Lateralisierung der fMRT-Daten und der Asymmetrie der DTI-Daten ( $p=0,046)$ (Tabelle 39).

Zur Überprüfung, ob sich in einem homogeneren Patientenkollektiv die Zusammenhänge zwischen funktioneller Lateralisierung und struktureller Asymmetrie anders darstellten, wurde die Subgruppe der Patienten mit ausschließlich linkshemisphärischer Epileptogenese (E links) mit Hilfe des Vorzeichentests untersucht. Die Signifikanzen änderten sich dabei im Vergleich zum Gesamtkollektiv der Epilepsiepatienten nicht. Die Auswertung des Sprachtests „Satzgenerierung“ ergab sowohl für das Broca-Areal $(p=0,505)$ als auch für das Wernicke-Areal $(p=1,000)$ zu einem Fehlerniveau von $5 \%$ keinen signifikanten Unterschied zwischen der funktionellen Lateralisierung und der strukturellen Asymmetrie. Gleiches galt für die ROls in der Broca-Region während des Sprachtests „Wortgenerierung“ $(p=0,505)$. Lediglich die Untersuchung der ROls in der Wernicke-Region zeigten während des Sprachtests "Wortgenerierung" einen signifikanten Unterschied $(p=0,041)$ hinsichtlich der Lateralisierung der fMRT-Daten und der Asymmetrie der weißen Substanz (Tabelle 39). 


\section{Diskussion}

Die vorliegende Arbeit untersuchte die Sprachlateralisierung und die strukturelle Asymmetrie der weißen Substanz sprachrelevanter Areale mit Hilfe nichtinvasiver MRMethoden. Patienten mit Temporallappenepilepsie wurden dafür mit einer gesunden Kontrollgruppe verglichen.

Eine exakte Identifizierung der sprachdominanten Hemisphäre ist z. B. in der präoperativen Diagnostik epilepsiechirurgischer Eingriffe bei Patienten mit pharmakoresistenter Temporallappenepilepsie von besonderer Bedeutung. Eine Alternative zum aktuellen Goldstandard, dem Wada-Test, könnten zukünftig bildgebende Verfahren wie fMRT und DTI darstellen.

Diese Arbeit untersuchte die Aktivierung der grauen Substanz und die hemisphärische Sprachlateralisierung mit Hilfe der fMRT. Ergänzend wurde die weiße Substanz mittels DTI auf eine strukturelle Asymmetrie in den sprachrelevanten Arealen untersucht. Die Kombination dieser beiden Verfahren erlaubte eine direkte Zuordnung struktureller Asymmetrie der weißen Substanz zur mittels fMRT bestimmten Sprachlateralisierung des Kortex.

Ziel war die Etablierung einer verlässlichen Methode zur Lokalisation sprachrelevanter Kortexareale durch den kombinierten Einsatz von nichtinvasiven MRT-Verfahren. Es wurde untersucht, ob sich die mittels fMRT detektierte Sprachlateralisierung auch in strukturellen Veränderungen der weißen Substanz widerspiegelt und DTI auf diese Weise zu einem Informationsgewinn bei der Lokalisation von Spracharealen führen könnte.

\subsection{Diskussion der fMRT-Ergebnisse}

Mit Hilfe eines in der fMRT-Forschung etablierten Verfahrens wurden zunächst die Aktivierung in der grauen Substanz sowie die Sprachlateralisierung bestimmt.

Die Auswertung der Sprachgenerierungstests während des fMRT-Versuchs beider Gruppen ergab, dass neben Aktivierung in den klassischen Brodmann-Arealen der Broca- und Wernicke-Region auch in benachbarten Regionen dieser beiden Zentren kortikale Aktivierung auftrat. Die Auswertung der ROls der Broca-Region zeigte Aktivitätsschwerpunkte in den BA 44 und 45, dem zytoarchitektonischen Korrelat der Broca-Region. Aktivitätsschwerpunkte in diesen Arealen wurden erwartet und sind ein Indiz für die Exaktheit der Methode. Darüber hinaus wurden Aktivitätsschwerpunkte in den benachbarten BA 6, 9, 13, 46 und 47 gefunden. Auch für die Wernicke-Region befanden sich während der Sprachtests die Aktivitätsschwerpunkte nicht nur in dem hinlänglich als zytoarchitektonisches Korrelat der Wernicke-Region bekannten BA 22, 
sondern auch in den benachbarten BA 13, 20, 21, 37, 41 und 42 (Abbildung 10). Die individuelle Betrachtung der einzelnen Aktivierungskarten zeigte darüber hinaus eine örtliche interindividuelle Variabilität der aktivierten Kortexareale.

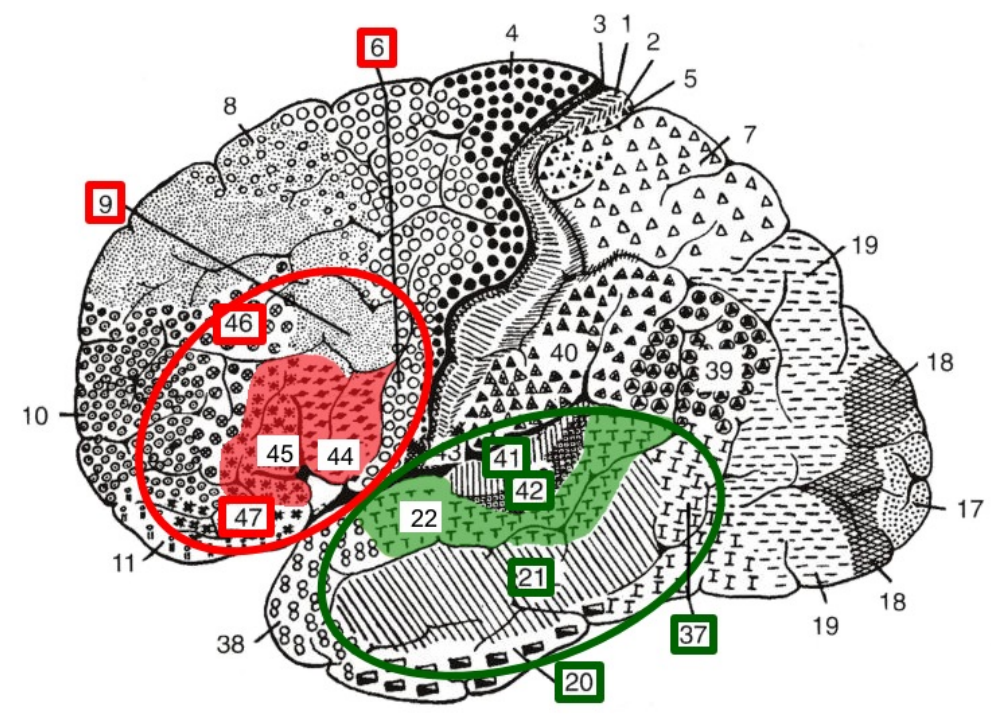

Abbildung 10: Lage der Aktivierungsschwerpunkte während der funktionellen Messungen in der Brodmann-Karte (Brodmann 1909, S. 131). Im Bereich der Broca-Region (rot) wurde Aktivierung in den BA 44 und 45 sowie in den benachbarten BA 6, 9, 46 und 47 nachgewiesen. Im Bereich der WernickeRegion (grün) wurde Aktivierung in dem BA 22 sowie in den benachbarten BA 20, 21, 37, 41 und 42 nachgewiesen. BA 13 ist als Bestandteil des Inselkortex nicht abgebildet.

Diese Ergebnisse zeigen, dass die kognitive Fähigkeit Sprache nicht streng an die klassischen Sprachzentren zytoarchitektonischer Definition gebunden ist, sondern auch benachbarte Regionen eine große Rolle spielen. Die vorliegenden Resultate bestätigten frühere fMRT-Studien, die kortikale Aktivierung in übereinstimmenden BA nachweisen konnten (Adcock et al. 2003; Binder et al. 1996; Stippich et al. 2003; Woermann et al. 2003).

Im Gegensatz zu vorausgegangenen Studien (Adcock et al. 2003; Binder et al. 1996; Lurito und Dzemidzic 2001; Spreer et al. 2002; Woermann et al. 2003) untersuchte die vorliegende Arbeit die Sprachlateralisierung jedoch allein mit nichtinvasiven bildgebenden Methoden. Ein Wada-Test (Wada und Rasmussen 1960), der den GoldStandard zur Untersuchung der Lateralität kognitiver Hirnleistungen darstellt, wurde nicht durchgeführt.

Der Wada-Test ist invasiv und belastend für den Patienten und birgt Risiken. Infolge der angiographischen Vorgehensweise können typische Komplikationen wie Nervenverletzungen an der Punktionsstelle, thromb-embolische Komplikationen oder eine Karotisdissektion auftreten. Darüber hinaus kann durch den konventionellen Wada-Test nur die relative Verteilung der Sprachfunktion zwischen den beiden Hemisphären bestimmt werden. Genauere Informationen über die Lokalisation der sprachrelevanten Areale innerhalb der Hemisphären sind mit dem herkömmlichen 
Wada-Test nicht zu erlangen. Gerade diese Informationen sind jedoch für die Planung eines eventuellen neurochirurgischen Eingriffs sehr wertvoll. Ein weiterer Schwachpunkt des konventionellen Wada-Tests ist seine Abhängigkeit von einer relativ getrennten und symmetrischen arteriellen Versorgung der Hemisphären. Eine atypische vaskuläre Versorgung der Hemisphären kann durch einen Querstrom zur simultanen Narkotisierung der kontralateralen Hirnhälfte führen und die Durchführung sowie die Auswertung des Wada-Tests dadurch beeinflussen (Hietala et al. 1990). Der selektive Wada-Test hingegen ermöglicht die Gewinnung von genaueren Informationen über die Funktion der zu resezierenden Hirnregionen, wie sie für die Operationsplanung nötig sind, und ist unabhängig von einer atypischen vaskulären Versorgung der Hemisphären. Diese Variante des Wada-Tests ist jedoch auch mit einem deutlich höheren Risiko für dauerhafte neurologische Defizite verbunden als der konventionelle Wada-Test (Urbach et al. 2002). Schließlich ist die Zeit zur Überprüfung bestimmter Hirnfunktionen, die dem Diagnostiker während des Wada-Tests bleibt, sehr begrenzt und eine wiederholte Testung aufgrund der Invasivität in der Regel nicht möglich (Binder et al. 1996; Spreer et al. 2002).

Die Etablierung einer nichtinvasiven bildgebenden Alternative zur Untersuchung der Sprachlateralisierung ist aufgrund der beschriebenen Schwächen des Wada-Tests von großer Bedeutung. FMRT und DTI könnten eine Alternative darstellen, deren Stärke in der möglichen Wiederholbarkeit der Messung, in der für den Untersucher ausreichend langen Zeit zur Bestimmung kortikaler Funktionen und in der fehlenden Beeinflussung der Testergebnisse durch die Variabilität des Blutflusses (Binder et al. 1996) liegt. Ein weiterer Vorteil von fMRT-gestützten Verfahren in Kombination mit anatomischen Daten stellt die Möglichkeit der genauen Lokalistation sprachrelevanter Kortexareale dar. Diese Informationen könnten zukünftig für die Planung von Resektionsgrenzen in der Epilepsiechirurgie von großem Nutzen sein.

Verschiedene Studien konnten bereits die Übereinstimmung der Ergebnisse des Wada-Tests mit den Ergebnissen von fMRT-Untersuchungen bezüglich der hemisphärischen Sprachlateralisierung zeigen (Adcock et al. 2003; Binder et al. 1996; Lurito und Dzemidzic 2001; Spreer et al. 2002; Woermann et al. 2003), so dass in der vorliegenden Studie auf einen Wada-Test verzichtet werden konnte. Die bisher erzielte Übereinstimmung von bis zu 90\% (Woermann et al. 2003) ist jedoch für eine präoperative Diagnostik noch nicht ausreichend hoch. Die Exaktheit und Zuverlässigkeit der nichtinvasiven Lateralitätsbestimmung sollte in der vorliegenden Studie durch verschiedene Faktoren optimiert werden.

Damit die vorliegende Arbeit trotz der individuellen Variabilität der Aktivierungskarten robuste und reproduzierbare Ergebnisse der Lateralitätsbestimmung kognitiver Funktionen gewährleisten konnte, erfolgte die Berechnung eines Lateralitätsindex. In der vorliegenden Arbeit wurde dabei auf die sorgfältige Auswahl der ROls und des Testparadigmas sowie auf die Anwendung streng standardisierter Auswertungsschritte 
geachtet, denn die Qualität eines Lateralitätsindex wird entscheidend durch diese Faktoren beeinflusst (Jansen et al. 2006; Lurito und Dzemidzic 2001).

Die Bestimmung der Lateralitätsindizes der fMRT-Daten zeigte, dass sowohl bei den gesunden Kontrollprobanden als auch bei den Patienten mit fokaler Epilepsie mehrheitlich eine linksseitige Sprachdominanz bestand (Abbildung 11).

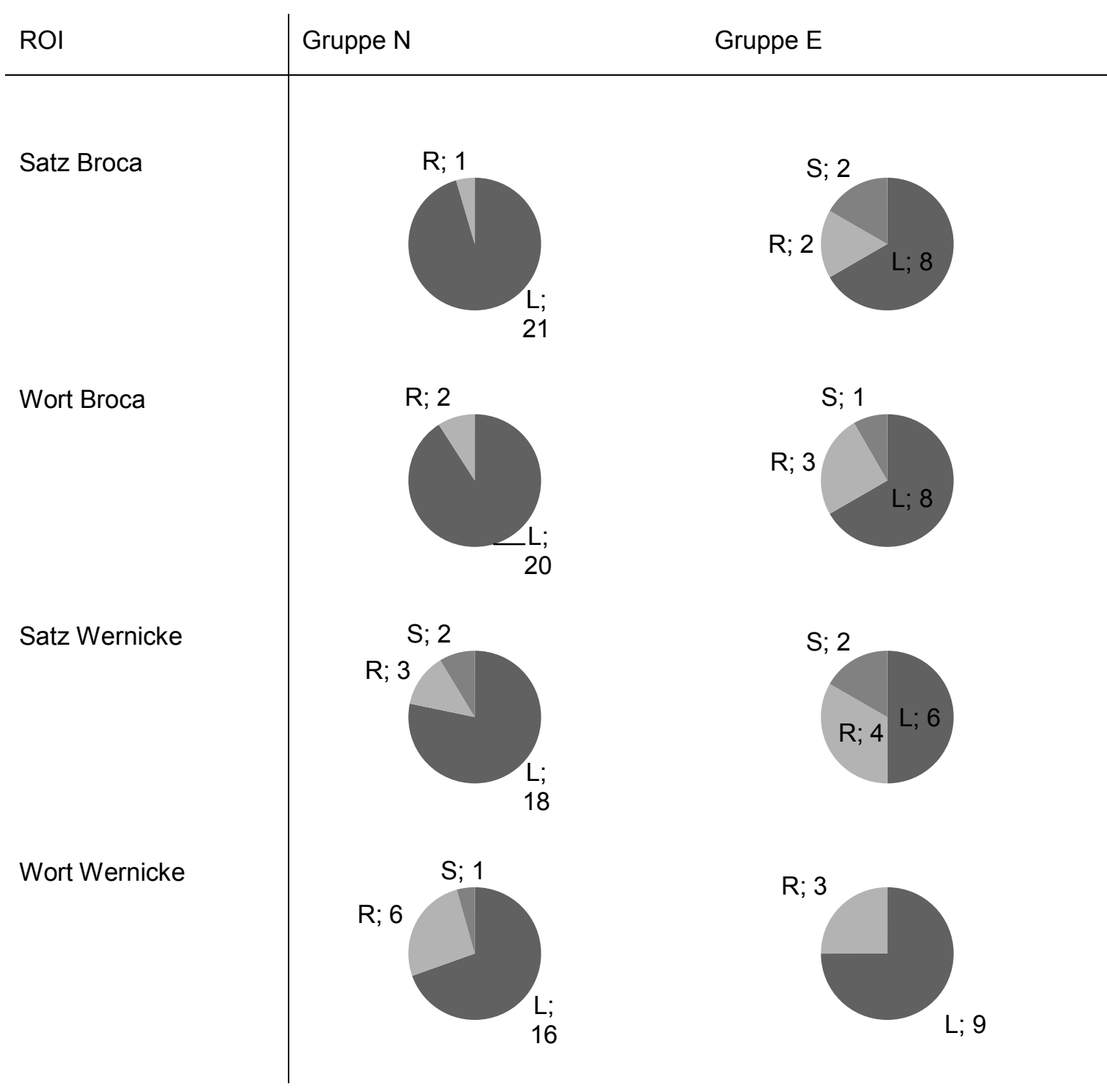

Abbildung 11: Verteilung der funktionellen Sprachlateralisierung in den einzelnen ROls für die Gruppen N und $\mathrm{E}$. L (dunkelgrau) = linkshemisphärische Sprachlateralisierung, R (hellgrau) = rechtshemisphärische Sprachlateralisierung, $\mathbf{S}$ (mittelgrau) $=$ symmetrische Sprachlateralisierung .

Die Ergebnisse der vorliegenden Studie bestätigten die Ergebnisse früherer fMRTStudien, die ebenfalls eine linkshemisphärische Dominanz sprachrelevanter Areale bei gesunden Rechtshändern und rechtshändigen Epilepsiepatienten zeigen konnten (Springer et al. 1999; Stippich et al. 2003; Woermann et al. 2003). 
In der vorliegenden Arbeit wurde ein in der Forschung zur Darstellung der Sprachregionen etabliertes fMRT-Versuchsprotokoll von Stippich et al. (2003) angewendet. Ziel der Studie von Stippich et al. (2003) war die Entwicklung eines verlässlichen fMRT-Protokolls zur präoperativen Bestimmung der Broca- und Wernicke-Region bei Patienten mit Hirnläsionen. Sie entwickelten ein fMRT-Protokoll, das die robuste Bestimmung der primären Sprachareale sowie die zuverlässige Bestimmung der Hemisphärendominanz der Sprache garantieren konnte. Das Protokoll untersuchte die Sprachfunktion mit Hilfe von Satzgenerierungs- und Wortgenerierungsaufgaben, welche zu einer spezifischen Aktivierung der Sprachareale führte, wohingegen motorische Kortexareale nicht aktiviert wurden. Stippich et al. (2003) konnten mit diesem fMRT-Protokoll verlässlich Aktivierung in den BA 44 und 45, die dem makroskopischen Korrelat der Broca-Region entsprechen, sowie dem BA 22 und den benachbarten BA 39 und 40, die dem makroskopischen Korrelat der Wernicke-Region entsprechen, nachweisen. Die untersuchte Probandengruppe bei Stippich et al. (2003) bestand jedoch ausschließlich aus gesunden Probanden. Die vorliegende Arbeit hingegen wendete dieses Versuchsprotokoll sowohl auf ein Kollektiv aus gesunden Kontrollprobanden als auch auf Patienten mit einer fokalen Epilepsie an. Die von Stippich et al. (2003) gefundenen Aktivierungsmuster konnten in der vorliegenden Arbeit für beide untersuchten Gruppen bestätigt werden. Dieses demonstriert, dass das verwendete fMRT-Protokoll auch bei Patienten mit einer fokalen Epilepsie verlässliche Daten ermittelt. Stippich et al. (2003) zeigten in ihrer Studie eine individuelle örtliche Variabilität der Sprachregionen und wiesen auf die Notwendigkeit der verlässlichen Lokalisation der individuell ausgeprägten Sprachregionen vor einem neurochirurgischen Eingriff am Gehirn hin. Auch die vorliegende Studie bestätigte diese Ergebnisse. Im Kollektiv der Patienten mit fokaler Epilepsie zeigte sich sogar eine interindividuelle höhere örtliche Variabilität der Sprachregionen. Dieses verdeutlicht den hohen Stellenwert einer exakten präoperativen Bestimmung der Sprachregionen bei Patienten mit fokaler Epilepsie.

Neben der mehrheitlich linksdominanten funktionellen Sprachlateralisierung zeigte der Vergleich der LIs des Kontrollkollektivs und des Patientenkollektivs unabhängig von dem durchgeführten Sprachtest für das Broca-Areal einen signifikanten Unterschied der Verteilung innerhalb der Gruppen (LIs der ROI Wort Broca: $p=0,041$; LIs der ROI Satz Broca: $p=0,0002$ ) (Tabelle 27). Die Gruppe der gesunden Kontrollprobanden zeigte häufiger eine linkshemisphärische Sprachdominanz als die Gruppe der Patienten mit fokaler Epilepsie. Die Lls der gesunden Kontrollprobanden mit linksdominanter Sprachlateralisierung zeigten eine stärkere Ausprägung im linksdominanten Bereich als die der Patienten mit Temporallappenepilepsie. In der Gruppe der Patienten mit fokaler Epilepsie trat hingegen häufiger eine bilaterale oder rechtshemisphärische und damit für Rechtshänder atypische Sprachdominanz auf. Die Patienten zeigten insgesamt eine stärkere Variabilität der Lls (Abbildung 12). 


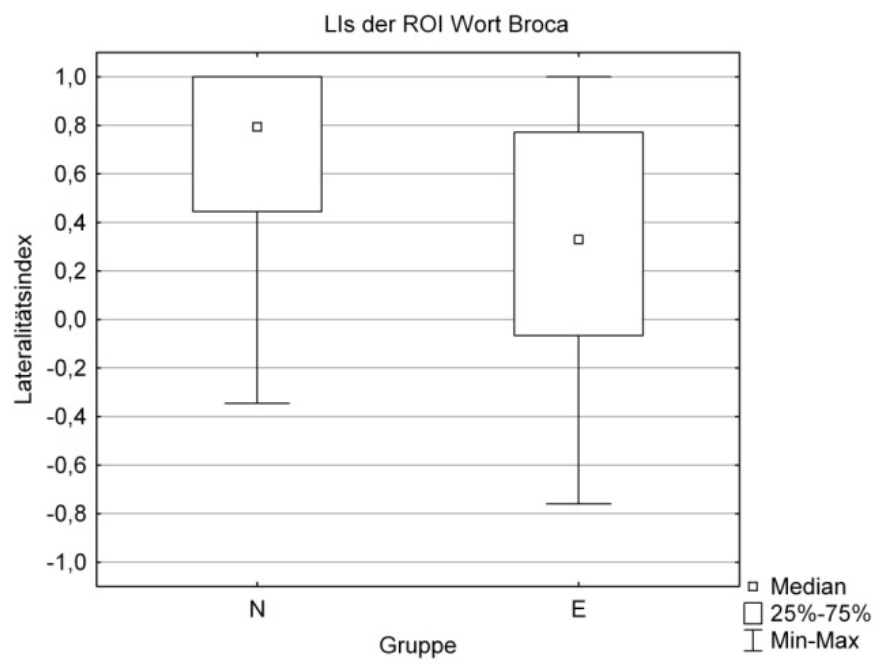

Gruppe N:

Maximum $=1$

Median $=0,793$

Minimum $=-0,346$

Gruppe E:

Maximum $=1$

Median $=0,329$

Minimum $=-0,760$

$p=0,041$

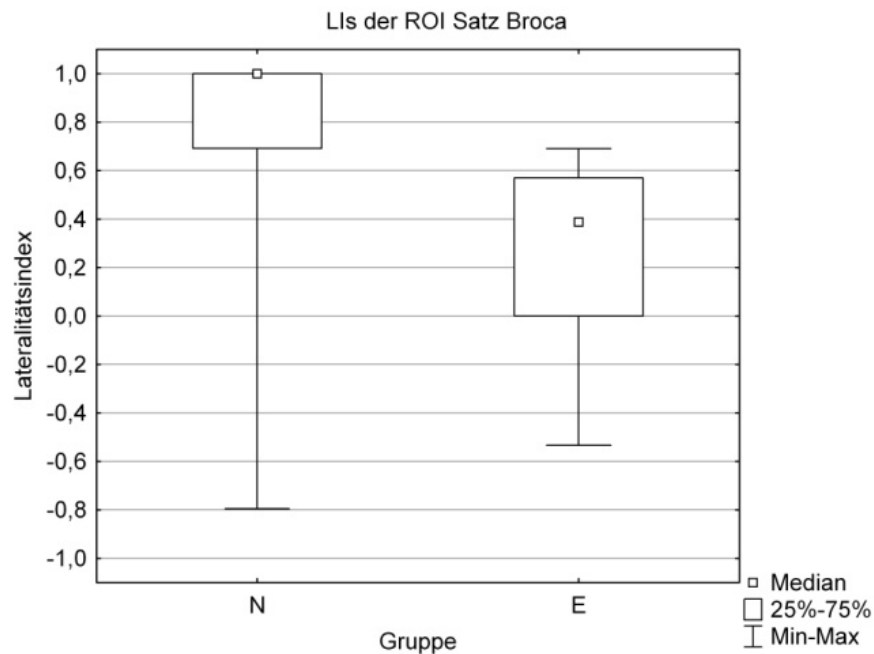

Gruppe N:

Maximum $=1$

Median $=1$

Minimum $=-0,795$

Gruppe E:

Maximum $=0,690$

Median $=0,388$

Minimum $=-0,534$

$p=0,0002$

Abbildung 12: Verteilung der LIs für die ROls in der Broca-Region in der Gruppe N ( $n=22)$ und $E(n=12)$. Der Kasten repräsentiert den Interquartilenabstand, die Antennen den Wertebreich zwischen Maximum und Minimum, das Quadrat den Median (Tabelle 23 und 25).

Für das Wernicke-Areal konnte kein signifikanter Unterschied hinsichtlich der Verteilung der LIs innerhalb der untersuchten Gruppen nachgewiesen werden (LIs der ROI Wort Wernicke: $p=0,605$; LIs der ROI Satz Wernicke: $p=0,164$ ) (Tabelle 27). Es zeigte sich jedoch die Tendenz, dass auch hier bei den untersuchten Patienten mit fokaler Epilepsie häufiger ein atypisches Aktivierungsmuster während der Sprachtests auftrat als bei den gesunden Kontrollprobanden (Abbildung 13). 


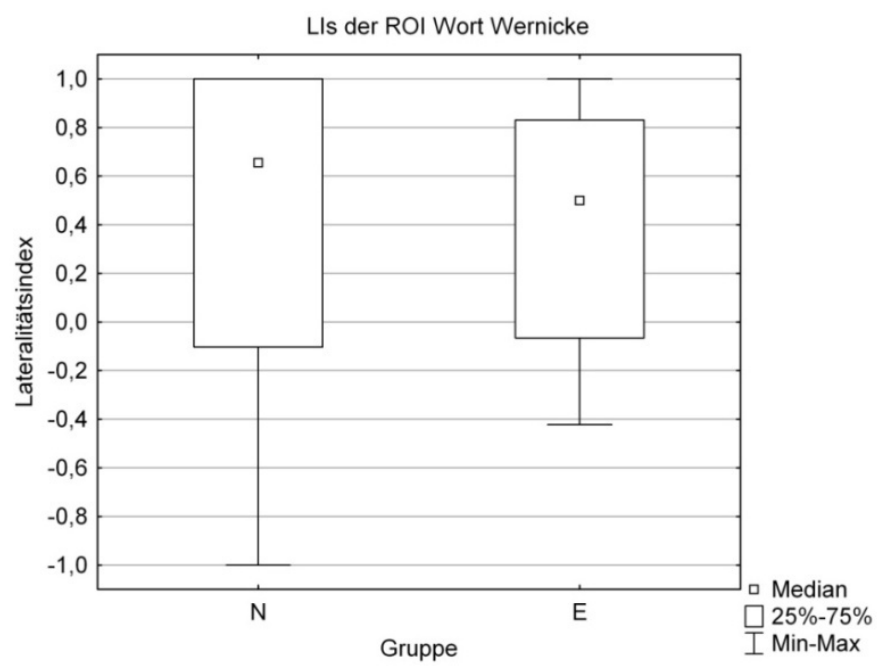

Gruppe N:

Maximum $=1$

Median $=0,655$

Minimum $=-1$

Gruppe E:

Maximum $=1$

Median $=0,499$

Minimum $=-0,423$

$p=0,605$

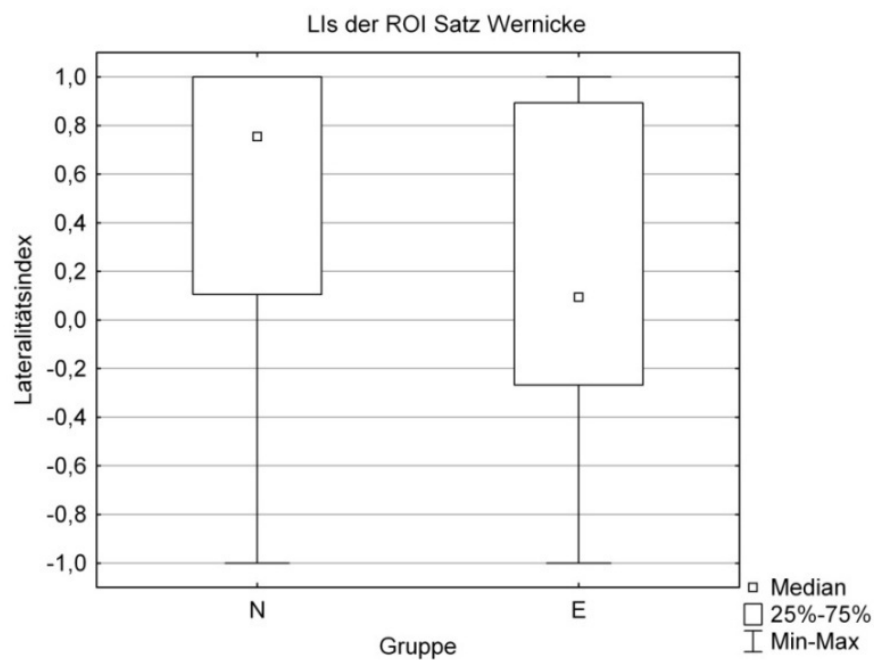

Gruppe N:

Maximum $=1$

Median $=0,755$

Minimum $=-1$

Gruppe E:

Maximum $=1$

Median $=0,094$

Minimum $=-1$

$p=0,164$

Abbildung 13: Verteilung der LIs für die ROls in der Wernicke-Region in der Gruppe $N(n=23)$ und $E$ $(n=12)$. Der Kasten repräsentiert den Interquartilenabstand, die Antennen den Wertebreich zwischen Maximum und Minimum, das Quadrat den Median (Tabelle 24 und 26).

Da das Patientenkollektiv in der vorliegenden Studie mehrheitlich jedoch nicht ausschließlich eine linkshemisphärische Epileptogenese aufwies, wurde zur Überprüfung der Ergebnisse das Kontrollkollektiv mit der Untergruppe der Patienten, die eine ausschließlich linkshemisphärische Epileptogenese aufwiesen, verglichen. In diesem Vergleich blieb im Bereich der Broca-Region ein signifikanter Unterschied in der Verteilung der LIs des Kontrollkollektivs und der LIs der Patienten mit ausschließlich linkshemisphärischer Epileptogenese bestehen (Lls der ROI Wort Broca: $p=0,018$; LIs der ROI Satz Broca: $p=0,001)$. Für die Wernicke-Region konnte hingegen trotz homogenem Patientenkollektiv mit ausschließlich linkshemisphärischer Epileptogenese im Vergleich mit dem Kontrollkollektiv kein signifikanter Unterschied hinsichtlich der Verteilung der LIs nachgewiesen werden (LIs der ROI Wort Wernicke: $p=0,801$; LIs der ROI Satz Wernicke: $p=0,346$ ) (Tabelle 28). Dieses zeigte, dass die drei Patienten, die keine linkshemisphärische Epileptogenese aufwiesen, die 
Auswertung der Ergebnisse nicht relevant beeinflussten. Für eine Gegenüberstellung mit den Patienten mit linkshemisphärischer Epileptogenese war ihre Anzahl jedoch zu gering.

Die gewonnen Ergebnisse unterstützen die Aussagen von Binder et al. (1996). Diese Gruppe demonstrierte, dass Patienten mit linkshemisphärischer Epileptogenese nicht immer eine eindeutige Sprachlateralisierung zeigen, und postulierte, dass Sprachlateralisierung nicht dichotom, sondern graduell zwischen den beiden Hemisphären verteilt ist. Sie untersuchte jedoch ausschließlich Patienten mit einer Epilepsie. Im Gegensatz zu Binder et al. (1996) führte die vorliegende Arbeit den Vergleich mit einer gesunden Kontrollgruppe. Es konnte eine signifikant verschiedene Sprachlateralisierung bei den untersuchten Patienten mit linkshemisphärischer fokaler Epilepsie gezeigt werden.

Springer et al. (1999) führten hingegen eine vergleichende fMRT-Studie zur Sprachlateralisierung bei gesunden Kontrollprobanden und Patienten, die an einer Epilepsie erkrankt waren, durch. Das Patientenkollektiv bestand dabei sowohl aus Patienten mit einer linksseitigen Temporallappenepilepsie (56\%) als auch aus Patienten mit einer rechtsseitigen Temporallappenepilepsie (44\%). Die Ergebnisse dieser Studie zeigten, dass in beiden Gruppen mehrheitlich eine linksdominante Sprachlateralisierung auftrat, die bei den Patienten unabhängig von der Hemisphäre des epileptogenen Fokus war. Des Weiteren bestand in der Gruppe der Epilepsiepatienten im Gegensatz zur Gruppe der gesunden Kontrollprobanden eine größere Variabilität in den Lls. Diese Ergebnisse waren jedoch bei Springer et al. (1999) nicht signifikant. Die fehlende Signifikanz könnte in dem verwendeten Versuchsprotokoll begründet sein. Springer et al. (1999) verwendeten eine semantische und auditive Entscheidungsaufgabe zur Bestimmung der Sprachlateralisierung. Die Diskriminierung wurde dabei über das Drücken eines Knopfes von den Probanden vorgenommen. Dieses Testparadigma führte zur Aktivierung einer Vielzahl kognitiver Systeme, unter anderem auch von nicht mit der Sprache assoziierten Kortexarealen, wie z.B. dem Motorkortex. Der LI wurde schließlich aus allen aktivierten Voxel der supratentoriellen lateralen zwei Drittel jeder Hemisphäre bestimmt. Diese rein anatomische Auswahl der ROI sowie die Miteinbeziehung der aktivierten Voxel des Motorkortex könnten zu einer ungenaueren Bestimmung der Sprachlateralisierung und damit zu der fehlenden Signifikanz zwischen den beiden untersuchten Gruppen geführt haben. Eine weitere Ursache für die fehlende Signifikanz könnte in dem gemischten Patientenkollektiv aus linksseitiger und rechtsseitiger Temporallappenepilepsie liegen. Im Gegensatz zu der Studie von Springer et al. (1999) konnte die vorliegende Arbeit für ein einheitlicheres Patientenkollektiv diese Ergebnisse nicht nur bestätigen, sondern zusätzlich einen signifikanten Unterschied in der Sprachlateralisierung zwischen den beiden untersuchten Gruppen nachweisen. 
Wie bereits erwähnt, spielt die Methode zur Bestimmung der ROI für die Exaktheit des Lateralitätsindex eine entscheidende Rolle und ist deshalb ein Qualitätsmerkmal für die Bestimmung der Sprachlateralisierung. Grundsätzlich kann eine ROI anhand anatomischer Orientierungspunkte (Binder et al. 1996; Springer et al. 1999) oder funktionell auf Basis von Aktivierungsmustern in fMRT-Karten (Adcock et al. 2003) definiert werden. Beide Auswertungsansätze zeigen Vor- und Nachteile.

Studien mit einem rein anatomischen Ansatz zur Bestimmung der Sprachlateralisierung (Binder et al. 1996; Springer et al. 1999) berechneten den LI z. B. auf Grundlage aller Voxel einer Hemisphäre oder aller aktivierten Voxel der supratentoriellen lateralen zwei Drittel jeder Hemisphäre. Auf der einen Seite führt solch eine rein anatomisch begründete Auswahl der ROI zu einer absolut objektiven Auswertung der Sprachlateralisierung. Auf der anderen Seite jedoch wird die individuelle Ausprägung der Sprachregionen in fMRT-Karten nicht berücksichtigt. Interessierende Aktivierung könnte außerhalb dieser anatomischen ROI liegen und damit nicht mit in die Auswertung einfließen. Darüber hinaus werden Regionen, die nicht durch das Experiment angesprochen werden, irrtümlicherweise mit in die Auswertung einbezogen und beeinflussen fälschlicherweise das Ergebnis. Aufgrund der genannten Nachteile wird die Sprachlateralisierung mit einer rein anatomischen Wahl der ROI nicht ausreichend exakt bestimmt.

Adcock et al. (2003) konnten in einer fMRT-Studie auf Grundlage von rein funktionell gewählten ROls übereinstimmend mit der vorliegenden Arbeit zeigen, dass Patienten mit einer fokalen Epilepsie, deren Fokus im linken Temporallappen liegt, signifikant weniger häufig eine linksdominante Sprachlateralisierung aufweisen. Solche rein funktionell gewählten ROls können unkompliziert nach den Vorgaben schon bestehender Studien erstellt werden. Hier liegt der Nachteil jedoch in der geringen Differenzierung des Aktivierungsstimulus. Häufig fließen Kortexregionen mit in die Auswertung ein, die nicht zu den interessierenden Gebieten gehören, wie z.B. der visuelle Kortex. Solche Rindengebiete zeigen jedoch häufiger eine symmetrische Aktivierung. In der Folge zeigen die Lls eine weniger klare als tatsächlich bestehende Lateralität an (Lurito und Dzemidzic 2001). Darüber hinaus lässt die manuelle Auswahl rein funktioneller ROls viel Raum für die Beeinflussung der Ergebnisse durch den Untersucher.

Auch Woermann et al. (2003) untersuchten die Sprachlateralisierung bei Epilepsiepatienten in einer der größten fMRT-Studien. Sie konnten eine $91 \%$ ige Übereinstimmung mit dem Ergebnis des Wada-Tests erreichen. Ein Schwachpunkt der Studie und möglicherweise Ursache dafür, dass keine höhere Konkordanz der beiden Verfahren zur Lateralitätsbestimmung erreicht wurde, lag jedoch in der Methode zur Bestimmung der Sprachlateralisierung. Die fMRT-Aktivierung in der rechten und der linken Hemisphäre wurde visuell auf Asymmetrie untersucht. Die Ergebnisse wurden 
daher stark durch die auswertende Person beeinflusst, so dass trotz Reproduzierbarkeit Zweifel an der Verlässlichkeit dieser Ergebnisse bestehen.

Im Gegensatz zu den oben genannten Studien verwendete die vorliegende Arbeit eine von Jansen et al. (2006) etablierte Methode zur Berechnung der Sprachlateralisierung. Jansen et al. (2006) verglichen anhand von zehn gesunden Kontrollprobanden verschiede Methoden zur Berechnung von Lateralitätsindizes auf der Basis von fMRTDaten. Für die Bestimmung der ROls wurde dabei von Jansen et al. (2006) eine kombinierte anatomische und funktionelle Vorgehensweise gewählt. Zunächst wurden, basierend auf den individuellen Aktivierungsmustern der fMRT-Karten, funktionelle ROls definiert. Diese wurden anschließend durch die Verwendung von neuroanatomischem Wissen in Regionen von Interesse, die z. B. fMRT-Aktivierung in der Broca-Region umfassten, und Regionen von Desinteresse, die z. B. fMRTAktivierung im visuellen Kortex umfassten, unterteilt. Jansen et al. (2006) konnten in ihrer Arbeit nachweisen, dass die Genauigkeit und Verlässlichkeit der Berechnung von LIs mit diesem Vorgehen am größten war. Analog zu der beschriebenen Methode von Jansen et al. (2006) diente in der vorliegenden Arbeit die anatomische Lokalisation der Broca- und Wernicke-Region als Anhaltspunkt zur Bestimmung der ROls. Hiervon ausgehend wurden diejenigen Aktivitätscluster als ROls definiert, die mit diesen anatomischen Landmarken am besten korrelierten. Diese kombinierte funktionelle und anatomische Methode erlaubte eine standardisierte Auswahl der ROls, die keinen Raum für bedienerabhängige Manipulation der Auswertungsschritte ließ und dennoch die individuellen Ausprägungen der funktionellen Kortexareale berücksichtigte.

Die Auswertung der fMRT-Daten der vorliegenden Arbeit konnte die Lokalisation sprachrelevanter Kortexareale sowohl bei der gesunden Kontrollgruppe als auch bei der Gruppe der Patienten mit fokaler Epilepsie mit der angewendeten Methode verlässlich darstellen.

Verschiedene Studien beschäftigten sich bereits mit der Frage, ob sich die funktionelle Sprachlateralisierung auch in einem strukturellen Korrelat der Hirnsubstanz widerspiegelt.

Im Rahmen dieser These untersuchten Amunts et al. (1999) den Gray level index (GLI) an postmortalen menschlichen Gehirnen, der das Verhältnis der Zellkörpermenge in einem definierten Kortexareal beschreibt. Sie entdeckten eine interhemisphärische Asymmetrie im BA 44 mit einem größeren GLI in der linken Hemisphäre als in der rechten Hemisphäre, welche ein strukturelles Korrelat der funktionellen Sprachlateralisierung darstellen könnte (Amunts et al. 1999).

Eine weitere post mortem Studie untersuchte die Rezeptorbesetzung und die Rezeptordichte verschiedener Neurotransmitter in der Broca-Region und in benachbarten Regionen menschlicher Gehirne (Amunts et al. 2010). In acht Gehirnen wurde die räumliche Verteilung von sechs verschiedenen Neurotransmitterrezeptoren 
im BA 44 und 45 analysiert. Die Broca-Region konnte auf diese Weise weiter aufgeteilt werden. Es zeigte sich, dass in der Broca-Region der linken Hemisphäre im Vergleich zur rechten Hemisphäre eine signifikant höhere absolute Rezeptordichte bestand. Die Asymmetrie der Rezeptordichte in der untersuchten grauen Substanz korrelierte dabei mit der linkshemisphärischen Sprachdominanz. Amunts et al. (2010) lieferten mit diesen Ergebnissen einen weiteren Hinweis für das Bestehen eines strukturellen Korrelats der Hirnsubstanz zur funktionellen Lateralisierung von Sprachproduktion.

Da es Hinweise auf strukturelle Veränderungen der grauen Substanz als Korrelat der funktionellen Sprachlateralisierung gibt, ist anzunehmen, dass auch die mit Sprache assoziierte weiße Substanz eine strukturelle Asymmetrie gleichsinnig zur funktionellen Sprachlateralisierung aufweist.

Die vorliegende Arbeit untersuchte die weiße Substanz mit Hilfe diffusionsgewichteter bildgebender Verfahren auf eine Asymmetrie. Dieses Verfahren ermöglichte im Gegensatz zu den zuvor genannten Studien erstmals eine in vivo-Beurteilung der Hirnstruktur von gesunden Probanden und Patienten mit einer Temporallappenepilepsie.

\subsection{Diskussion der DTI-Ergebnisse}

Die vorliegende Arbeit untersuchte die weiße Substanz mit Hilfe von diffusionsgewichteten MRT-Messungen auf eine Asymmetrie der FA und lieferte somit Informationen über das Diffusionsverhalten in der mit den Spracharealen assoziierten weißen Substanz. Die Diffusion war dabei in Faserbündeln der weißen Substanz aufgrund vieler paralleler Diffusionsbarrieren stärker gerichtet als in der grauen Substanz, die viele Zellkörper, jedoch wenige parallele Diffusionsbarrieren aufwies. Höhere FA-Werte waren ein Hinweis auf stärker gerichtete Diffusion in den betrachteten Voxeln. Dieses Phänomen konnte in einem Asymmetrieindex (AI) quantifiziert werden.

Auch in der Auswertung der DTI-Daten sollte analog zur Auswertung der fMRT-Daten ein Verfahren zur Bestimmung der FA angewendet werden, das den geringstmöglichen Raum für willkürliche und untersucherabhängige Auswertungsschritte ließ. Hierfür wurden die bereits in der Auswertung der fMRT-Daten bestimmten ROls als Orientierungspunkte genutzt und anschließend die mit diesen ROls korrelierende weiße Substanz zur Auswertung der FA verwendet.

Die auf diese Weise ermittelten Aussagen über die Struktur der weißen Substanz spiegelten die Ergebnisse der fMRT-Daten wider.

Für das Broca-Areal konnte nachgewiesen werden, dass sich die Verteilung der Als zwischen den Gruppen signifikant voneinander unterschieden (Als der ROI Wort 
Broca: $p=0,017$; Als der ROI Satz Broca: $p=0,0002$ ) (Tabelle 37). Dabei zeigten die gesunden Kontrollprobanden mehrheitlich eine Asymmetrie mit linksseitig höheren FAWerten im Gegensatz zur Gruppe der Patienten mit fokaler Epilepsie. Darüber hinaus zeigten die Als der gesunden Kontrollprobanden eine Asymmetrie mit linkshemisphärisch positiveren FA-Werten als die Gruppe der Patienten mit Temporallappenepilepsie. Bei den Patienten war eine stärkere Variabilität der linksgerichteten Als zu beobachten. Außerdem fiel in der Patientengruppe deutlich häufiger eine symmetrische oder überwiegend rechtsgerichtete und damit atypische Verteilung der Al auf (Abbildung 14).

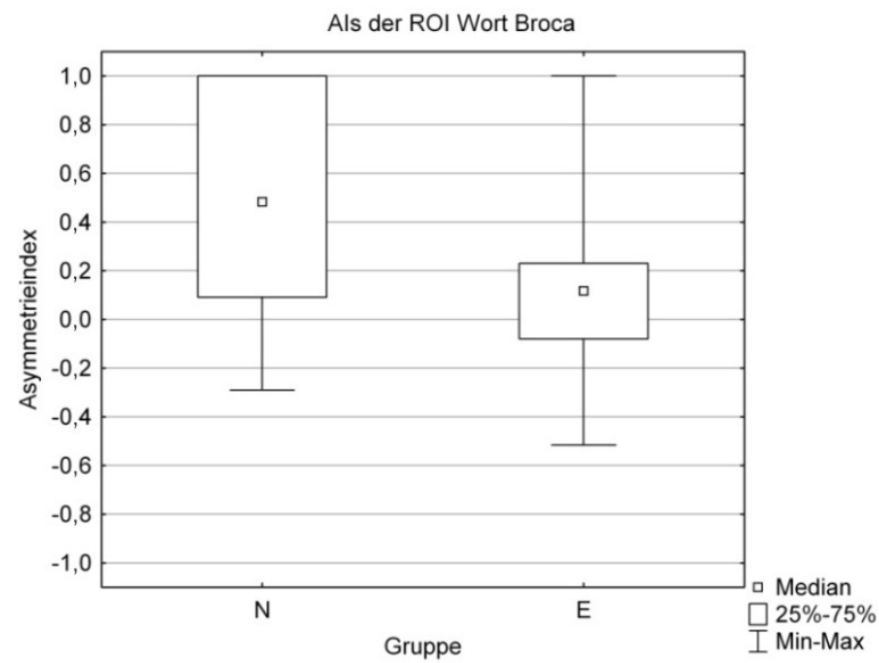

Gruppe N:

Maximum $=1$

Median $=0,483$

Minimum $=-0,290$

Gruppe E:

Maximum $=1$

Median $=0,117$

Minimum $=-0,516$

$p=0,017$

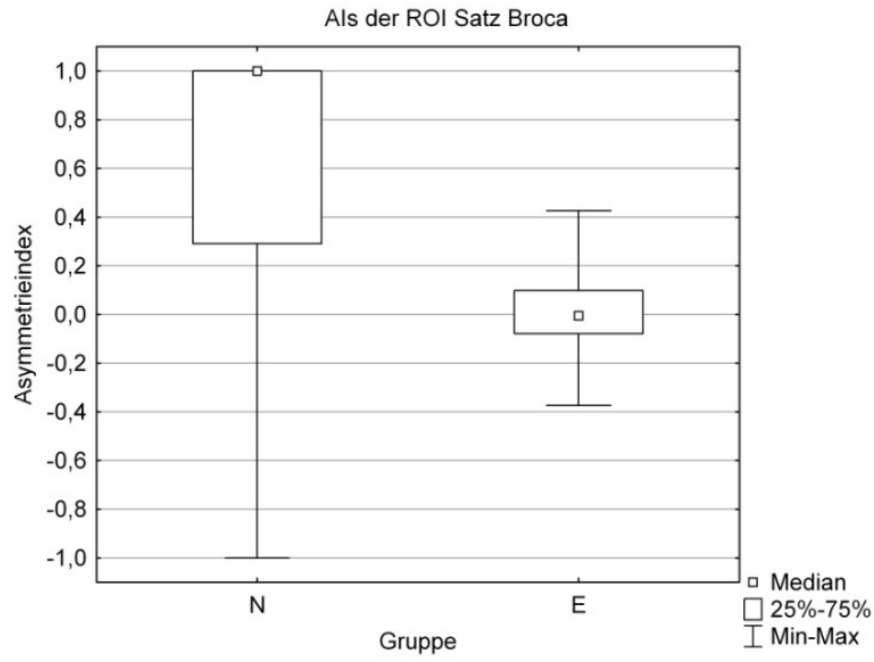

Gruppe N:

Maximum $=1$

Median $=1$

Minimum $=-1$

Gruppe E:

Maximum $=0,426$

Median $=-0,005$

Minimum $=-0,374$

$p=0,0002$

Abbildung 14: Verteilung der Als für die strukturellen ROls in der Broca-Region in der Gruppe $N(n=23)$ und $E(n=12)$. Der Kasten repräsentiert den Interquartilenabstand, die Antennen den Wertebereich zwischen Maximum und Minimum, das Quadrat den Median (Tabelle 33 und 34).

Im Wernicke-Areal hingegen ergab sich keine signifikant unterschiedliche Verteilung der Als zwischen den beiden Gruppen (Als der ROI Wort Wernicke: $p=0,332$; Als der ROI Satz Wernicke: $p=0,175$ ) (Tabelle 37). Dennoch fiel auf, dass in der gesunden Kontrollgruppe häufiger eine linksgerichtete Asymmetrie der FA-Werte bestand, 
während in der Gruppe der Patienten mit Temporallappenepilepsie häufiger eine symmetrische oder rechtshemisphärische Verteilung der FA auftrat. Somit wurde auch für das Wernicke-Areal, wenn auch nicht signifikant, eine größere Streuung der Als im Patientenkollektiv beobachtet (Abbildung 15).

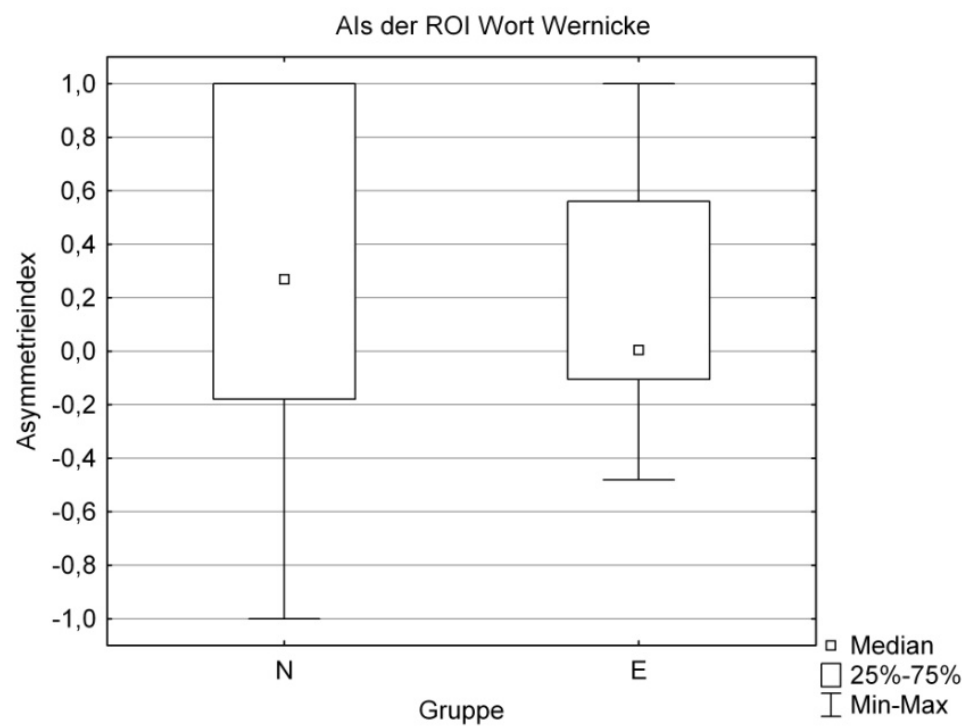

Gruppe N:

Maximum $=1$

Median $=0,269$

Minimum $=-1$

Gruppe E:

Maximum $=1$

Median $=0,005$

Minimum $=-0,481$

$p=0,332$

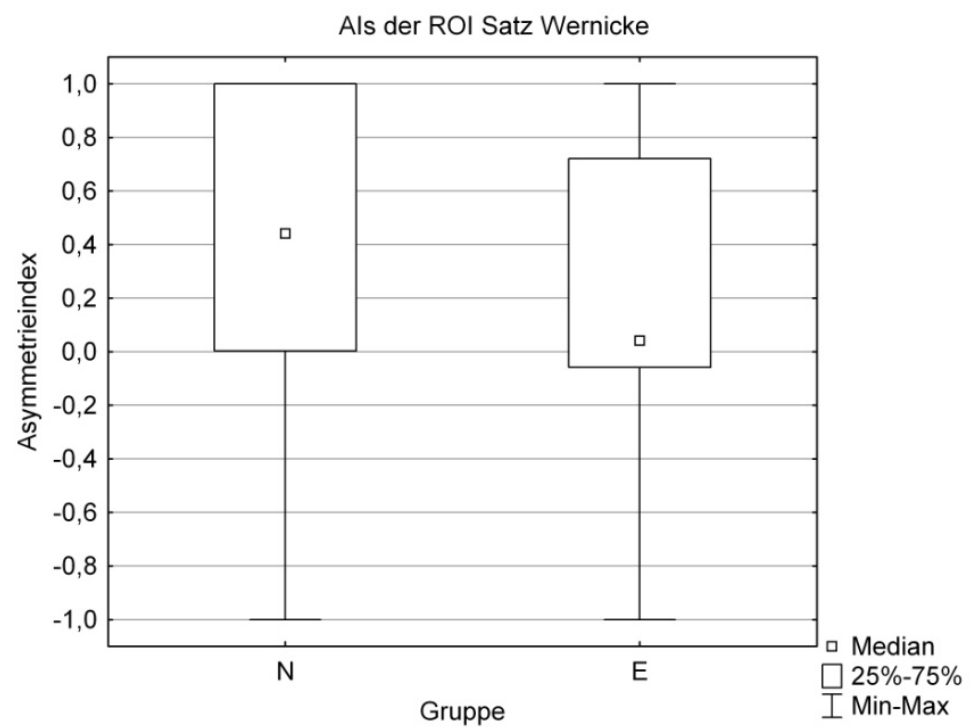

Gruppe N:

Maximum $=1$

Median $=0,441$

Minimum $=-1$

Gruppe E:

Maximum $=1$

Median $=0,042$

Minimum $=-1$

$p=0,175$

Abbildung 15: Verteilung der Als für die strukturellen ROls in der Wernicke-Region in der Gruppe N $(n=23)$ und $E(n=12)$. Der Kasten repräsentiert den Interquartilenabstand, die Antennen den Wertebereich zwischen Maximum und Minimum, das Quadrat den Median (Tabelle 35 und 36).

Wie bereits erwähnt, war die Epileptogenese in dem untersuchten Patientenkollektiv mehrheitlich, allerdings nicht ausschließlich linkshemisphärisch lokalisiert. Zur Überprüfung, ob sich die Ergebnisse in einem Patientenkollektiv mit einer homogenen Epileptogenese veränderten, wurde das Kontrollkollektiv mit der Untergruppe der Patienten, die eine ausschließlich linkshemisphärische Epileptogenese aufwiesen, verglichen. Dieser Vergleich zeigte weiterhin im Bereich der Broca-Region einen 
signifikanten Unterschied in der Verteilung der Als des Kontrollkollektivs und der Als der Patienten mit ausschließlich linkshemisphärischer Epileptogenese (Als der ROI Wort Broca: $p=0,012$; Als der ROI Satz Broca: $p=0,002)$. Für die Wernicke-Region konnte hingegen trotz homogenem Patientenkollektiv mit ausschließlich linkshemisphärischer Epileptogenese im Vergleich mit dem Kontrollkollektiv kein signifikanter Unterschied hinsichtlich der Verteilung der Als nachgewiesen werden (Als der ROI Wort Wernicke: $p=0,753$; LIs der ROI Satz Wernicke: $p=0,304$ ) (Tabelle 38). Die Ergebnisse waren ein Indiz dafür, dass die drei Patienten mit nicht linkshemisphärischer Epileptogenese die Auswertung nicht relevant beeinflussten. Aufgrund ihrer geringen Anzahl wurde auf eine Gegenüberstellung mit den Patienten mit linkshemisphärischer Epileptogenese verzichtet.

Zur Überprüfung, ob sich eine funktionelle Sprachlateralisierung in einer strukturellen Asymmetrie der weißen Substanz widerspiegelte, wurde die Verteilung der LIs und der Als mir Hilfe des Vorzeichentests miteinander verglichen.

In der Kontrollgruppe konnten für keine der untersuchten ROls signifikante Unterschiede zwischen der funktionellen Lateralisierung der fMRT-Daten und der strukturellen Asymmetrie der DTI-Daten nachgewiesen werden. Die Hemisphäre mit den höheren FA-Werten entsprach in dieser Gruppe der Hemisphäre der funktionellen Sprachlateralisierung. In der Hemisphäre der funktionellen Sprachdominanz zeigten sich stärker gerichtete Diffusionseigenschaften der mit den Sprachzentren assoziierten weißen Substanz als in der nicht dominanten Hemisphäre, welche als Korrelat für die veränderte Mikrostruktur in den untersuchten ROls gewertet werden kann.

Auch in der Gruppe der Patienten mit Temporallappenepilepsie entsprachen die Ergebnisse der funktionellen Lateralisierung weitgehend denen der strukturellen Asymmetrie. Für die ROls in der Broca-Region sowie die ROls im Wernicke-Areal, die während des funktionellen Sprachtests "Satzgenerierung“ definiert wurden, zeigten sich keine signifikanten Unterschiede zwischen der funktionellen Lateralisierung und der strukturellen Asymmetrie in den zugehörigen strukturellen ROls. In diesen ROIs entsprach die funktionelle Sprachlateralisierung einer höheren Gerichtetheit der Diffusion. Einzig für die ROI „Wort Wernicke“ konnte im Patientenkollektiv keine Korrelation zwischen funktionellen Lls und strukturellen Als nachgewiesen werden. Die Verteilung der LIs der fMRT-Daten und der Als der DTI-Daten unterschied sich in dieser ROI im Patientenkollektiv signifikant $(p=0,046)$ (Tabelle 39) voneinander. Eine mögliche Erklärung könnte die Lokalisation der Epileptogenese der untersuchten Patienten sein. Diese wiesen mehrheitlich eine linkshemisphärische Temporallappenepilepsie auf. Aufgrund der pathologischen Prozesse war es möglicherweise in der Broca-Region zu einer stärkeren Reorganisation von Funktion und Anatomie gekommen als in der Wernicke-Region, die nicht so stark beeinflusst schien. 
Die Ergebnisse der vorliegenden Arbeit zeigen, dass sich eine funktionelle Sprachlateralisierung sowohl bei Patienten mit einer fokalen Epilepsie als auch bei gesunden Probanden in einer Asymmetrie der FA der mit den Sprachzentren assoziierten weißen Substanz widerspiegelt. Die These, dass funktioneller Sprachlateralisierung eine Asymmetrie der Mikrostruktur der weißen Substanz zugrunde liegt, konnte die vorliegende Arbeit bestätigen. Die Sprachlateralisierung scheint dabei in der Broca-Region stärker ausgebildet zu sein als in der WernickeRegion.

Auch verschiedene andere Studiengruppen beschäftigten sich bereits mit der Frage, ob sich funktionelle Lateralisierung des Gehirns in struktureller Asymmetrie widerspiegelt.

Good et al. (2001) analysierten die Asymmetrie der grauen und weißen Substanz mit Hilfe einer Voxel-basierten morphometrischen Untersuchung. Neben einer linkshemisphärischen Volumensteigerung der grauen Substanz in sprachassoziierten Arealen des Temporallappens zeigte auch die benachbarte weiße Substanz eine asymmetrische Volumensteigerung im linken Temporallappen. Diese Erkenntnisse zur Asymmetrie der weißen Substanz unterstützen die These der vorliegenden Arbeit, dass ein strukturelles Korrelat der funktionellen Sprachlateralisierung besteht. Die von Good et al. (2001) gewählte Voxel-basierte morphometrische Gruppenanalyse ermöglichte jedoch allein quantitative Aussagen über die untersuchte Substanz. Informationen über die Qualität der Diffusionseigenschaften und damit über die Mikrostruktur der untersuchten Hirnsubstanz konnten nicht gewonnen werden. Darüber hinaus erlaubte die Gruppenanalyse der Probanden keine Aussagen zur Hirnstruktur einzelner Individuen, obwohl insbesondere die kognitive Funktion Sprache ein individuelles Muster der sprachassoziierten Strukturen zeigt. Ein weiterer Kritikpunkt ist, dass Good et al. (2001) nur gesunde Probanden untersuchten. Ob diese Methode auch im klinischen Altag bei Patienten mit z. B. einer Epilepsie angewendet werden kann, konnte diese Studie nicht beantworten. Die vorliegende Arbeit hingegen verglich die Diffusionseigenschaften der weißen Substanz von gesunden Kontrollprobanden und von Patienten mit einer Temporallappenepilepsie. Die verwendete DTI-Methode konnte sowohl für die Kontrollprobanden als auch für die Patienten mit einer Temporallappenepilepsie qualitative Aussagen über die Diffusionseigenschaften und damit auch über die Mikrostruktur der weißen Substanz auf individueller Ebene machen.

Briellmann et al. (2003) untersuchten mit einem DTI-Verfahren Patienten mit fokaler Epilepsie, deren Epileptogenese im linksseitigen Temporallappen lag. Über die quantitative Bestimmung der FA-Werte in definierten ROls und die Bildung eines Als konnten sie nachweisen, dass eine verminderte linksseitige Anisotropie bei diesen Patienten mit einer verminderten linkslateralisierten Sprachfunktion einherging. Obwohl die Patienten mit fokaler Epilepsie häufiger eine atypische kortikale Sprachorganisation 
aufwiesen als die gesunden Kontrollprobanden, korrelierte die funktionelle Sprachlateralisierung mit der Asymmetrie der FA. Die vorliegende Arbeit konnte diese Ergebnisse bestätigen. Die Betrachtung der individuellen Als zeigte, dass diejenigen Patienten, die keine linkshemisphärische Sprachlateralisierung und damit eine atypische Sprachlateralisierung zeigten, auch eine linkshemisphärisch geringere Anisotropie der DTI-Daten in den untersuchten ROls aufwiesen (Tabelle 7, 12, 17, 22, 29, 30, 31 und 32). Auch in der vorliegenden Arbeit ging die funktionelle Sprachlateralisierung mit einer veränderten Mikrostruktur der weißen Substanz einher. Die Ergebnisse von Briellmann et al. (2003) beruhten jedoch auf ROls der DTI-Karten, die auf Basis von anatomischen Landmarken manuell in die weiße Substanz gelegt wurden. Die Ergebnisse der Berechnungen dieser ROls wurden anschließend mit der funktionellen Sprachlateralisierung verglichen, die auf Grundlage aller signifikant aktivierten ROls einer Hemisphäre berechnet wurden. Eine direkte Zuordnung von Funktion und Struktur ist durch diese unterschiedliche Art der ROI-Definition in den funktionellen und strukturellen Karten nicht möglich. Die vorliegende Arbeit hingegen untersuchte die weiße Substanz, die direkt in Verbindung mit den funktionellen ROls der grauen Substanz stand. Die Definition der ROIs beruhte dabei nicht auf manuellen und damit durch den Auswerter manipulierbaren Entscheidungen. Diese Methode ermöglichte exakte und reproduzierbare Ergebnisse über die direkten Zusammenhänge zwischen Sprachfunktion und Mikrostruktur.

Eine weitere Methode zur Untersuchung der Struktur der weißen Substanz ist die Traktographie. Dafür wird die FA bestimmter Faserverläufe der weißen Substanz analysiert und die Faserverläufe werden rekonstruiert. Viele Studien untersuchen die FA frontotemporaler Faserbündel zwischen der Broca- und der Wernicke-Region wie dem Fasciculus longitudinalis superior (superior longitudinal fasciculus $=$ SLF) und dem Fasciculus arcuatus (arcuate fasciclus = AF), da angenommen wird, dass diese Faserbündel die Broca- mit der Wernicke-Region verbinden und einen wichtigen Bestandteil des Sprachnetzwerkes bilden (Geschwind 1970; Makris et al. 2005).

Powell et al. (2006) konnten durch die Kombination von fMRT und Traktographie des SLF bei Rechtshändern eine signifikante Korrelation von struktureller Asymmetrie und funktioneller Sprachlateralisierung nachweisen. Eine stärkere linksseitige Sprachlateralisierung der fMRT-Daten ging mit erhöhter linksseitiger FA im SLF einher. Diese Ergebnisse lassen vermuten, dass strukturelle Asymmetrien der FA von Faserverläufen die Grundlage der funktionellen Sprachlateralisierung bilden. Doch Powell et al. (2006) untersuchten einzig gesunde Rechtshänder mit typischer Sprachlateralisierung. Informationen über die Anwendbarkeit der Methode und die Ergebnisse zur Mikrostruktur bei atypischer Sparchlateralisierung, wie sie häufig bei neurologischen Patienten vorkommt, konnte diese Studiengruppe im Gegensatz zur vorliegenden Arbeit nicht gewinnen. 
Vernooij et al. (2007) hingegen untersuchten neben gesunden Rechtshändern auch gesunde Linkshänder, die eine atypische Sprachlateralisierung aufwiesen. Diese Gruppe wies in einer kombinierten DTI- und fMRT-Studie mittels Traktographie des AF eine linksseitig höhere relative Faserdichte des AF nach, die unabhängig von Händigkeit oder funktioneller Sprachlateralisierung bestand. Diese Studie stellte die These in Frage, dass eine funktionelle Sprachlateralisierung mit einer strukturellen Asymmetrie im AF einhergeht.

Weitere Traktographiestudien untersuchten die atypische Sprachlateralisierung bei Patienten mit fokaler Epilepsie. Rodrigo et al. (2008) zeigten eine Korrelation von struktureller Asymmetrie des AF mit linksseitiger funktioneller Sprachlateralisierung bei Patienten mit einer rechtsseitigen Temporallappenepilepsie. Eine stärkere Sprachlateralisierung ging in dieser Studie mit einer höheren Asymmetrie der FA-Werte einher. Allerdings konnte diese Korrelation nicht für die untersuchten Patienten mit einer linksseitigen Temporallappenepilepsie bestätigt werden.

Eine aktuellere Studie hingegen konnte sowohl für Patienten mit einer linksseitigen als auch mit einer rechtsseitigen Epileptogenese eine hohe Korrelation zwischen der funktionellen Sprachlateralisierung im fMRT-Versuch und der Asymmetrie der FA des AF im DTI-Versuch nachweisen (Ellmore et al. 2010). Die sprachdominante Hemisphäre bei Epilepsiepatienten verfügte dabei über signifikant höhere FA-Werte im untersuchten AF als die nichtdominante Hemisphäre. Ellmore et al. (2010) postulierten, dass auch die Mikrostruktur der weißen Substanz eine mit der funktionellen Sprachlateralisierung korrelierende Seitendifferenz aufweise. Ein Al, basierend auf den Werten der FA, ließ verlässliche Aussagen über die Sprachdominanz der Hemisphären zu. In Kombination mit fMRT-Daten konnte eine exakte Identifikation der sprachdominanten Hemisphäre gewährleistet werden.

Eine methodische Schwachstelle der oben genannten Traktographie-Studien (Ellmore et al. 2010; Rodrigo et al. 2008; Vernooij et al. 2007) ist jedoch das Problem der kreuzenden Fasern. Bei der Traktographie konnten nur diejenigen Faserverläufe beurteilt werden, die durch die definierten Start- und Endregionen verliefen. Sich in einem Voxel kreuzende Fasern konnten von diesem Algorithmus nicht berücksichtigt werden und verfälschten das Ergebnis. Darüber hinaus wurden die Start- und Endregionen der Traktographie in den genannten Studien anhand von anatomischen Orientierungspunkten ohne Berücksichtigung der funktionellen Sprachaktivierung gesetzt. Diese Vorgehensweise könnte eine Erklärung für die zum Teil fehlende Korrelation von funktioneller Sprachlateralisierung und struktureller Asymmetrie der weißen Substanz darstellen.

Darüber hinaus untersuchten die oben genannten Studien ausgewählte Faserstränge wie den SLF oder den AF mittels Traktographie. Gegensätzliche DTI-Ergebnisse bezüglich der Asymmetrie in diesen Strukturen lassen Zweifel daran aufkommen, dass 
der SLF oder der AF allein durch die kognitive Funktion Sprache beeinflusst wird. Es ist bekannt, dass der SLF aus vier verschiedenen Komponenten besteht, unter anderem dem AF, und nicht nur für die Sprachverarbeitung eine Rolle spielt, sondern auch für andere höhere kognitive Funktionen wie z. B. Gedächtnis und Aufmerksamkeit (Makris et al. 2005). Auch die Rolle des AF in der Sprachverarbeitung ist nicht absolut eindeutig, da er eine große Anzahl sensorischer Kortexareale mit sensorischen und präfrontalen Kortexarealen verbindet (Dronkers et al. 2000). Es besteht daher die Möglichkeit, dass der SLF und der AF auch für andere kognitive Fähigkeiten, die unabhängig von der funktionellen Sprachlateralisierung eine linksseitige Lateralisierung aufweisen, eine wichtige Rolle spielen und dieses die Ergebnisse beeinflusste. Daher scheinen diese Strukturen nicht optimal geeignet zu sein, um Veränderungen in der mit Sprache assoziierten weißen Substanz zu detektieren.

Die vorliegende Studie konnte nachweisen, dass die funktionelle Sprachateralisierung mit einer veränderten Mikrostruktur der weißen Substanz einhergeht. Dieser Zusammenhang bestand auch bei atypischer Sprachlateralisierung, wie sie z. B. bei Patienten mit fokaler Epilepsie vorkommt. Die Gleichsinnigkeit der atypischen Sprachlateralisation und der Asymmetrie der FA im untersuchten Patientenkollektiv gibt Hinweise auf das Vorliegen neuroplastischer Umbauvorgänge der weißen Substanz, die durch eine fokale Epilepsie induziert werden.

Eine mögliche Erklärung für die nachgewiesenen Veränderungen in der weißen Substanz bei Patienten mit fokaler Epilepsie liegt in der Ätiologie der Erkrankung. Die Hippokampussklerose stellt die häufigste Ursache einer Temporallappenepilepsie dar (Mathern et al. 1997). Im Tiermodell konnte bereits für diese Erkrankung eine strukturelle Reorganisation der Hirnsubstanz, insbesondere des Hippokampus, nachgewiesen werden (Okazaki et al. 1995). Neuere DTI-Verfahren ermöglichen die indirekte Darstellung der menschlichen Hirnsubstanz und erlauben Untersuchungen der Mikrostruktur der weißen Substanz.

DTI-Studien mit Patienten, die an einer Temporallappenepilepsie leiden, konnten bereits zeigen, dass im Hippokampus der Hemisphäre des epileptogenen Fokus im Vergleich zum kontralateralen Hippokampus das Diffusionsvermögen erhöht (Yoo et al. 2002) und die FA vermindert ist (Assaf et al. 2003). Diese Veränderungen des Diffusionsvermögens und der FA sind indirekte Zeichen für eine veränderte Mikrostruktur, wie sie z. B. durch Interneuronenverluste und Neurogenese von Progenitor-Zellen im Hippokampus entstehen könnte. Beide Phänomene sind vermutlich für die Entstehung von fokalen Epilepsien verantwortlich (Scharfman et al. 2000; Sloviter 1987).

Veränderungen der Mikrostruktur der weißen Substanz konnten auch in subkortikalen Regionen, die über den epileptogenen Fokus hinausgehen, beobachtet werden (Concha et al. 2009; Thivard et al. 2005) und führten zu der Annahme, dass die 
Epileptogenese auch Einfluss auf die Mikrostruktur der verbindenden weißen Substanz hat. Die vorliegende Arbeit konnte ebenfalls Veränderungen in der Mikrostruktur der weißen Substanz nachweisen, die nicht direkt mit dem epileptogenen Fokus übereinstimmte, jedoch mit den funktionellen Sprachaktivierungsmustern in enger Beziehung stand. Eine mögliche Erklärung für diese Ergebnisse ist die Propagation von epileptischen Anfällen in Fokus ferne Regionen, die auch zu Veränderungen der Mikrostruktur der verbindenden weißen Substanz führen könnte.

FA-Karten können die Ausrichtung und die Intaktheit von neuronalen Fasern visualisieren. Die FA wird dabei entscheidend durch die Ausrichtung der Myelinscheiden und der axonalen Membranen der Fasern in der weißen Substanz beeinflusst, so dass ein Verlust der longitudinalen Ausrichtung zu einer Verminderung der FA führt (Beaulieu 2002).

Die vorliegende Arbeit postuliert, dass im Umkehrschluss die im Seitenvergleich erhöhte FA in der mit den dominanten Spracharealen assoziierten weißen Substanz durch eine stärkere Gerichtetheit und eine erhöhte Axondichte bedingt ist. Diese Arbeit bestätigt die These, dass eine aufgrund der Epileptogenese atypische funktionelle Sprachlokalisation mit Auswirkungen auf die Mikrostruktur der weißen Substanz, die für Reizweiterleitung zuständig ist, einhergeht.

Diese gleichsinnige Veränderung von Aktivität und Mikrostruktur bietet neue Möglichkeiten zur exakten Lokalisation sprachrelevanter Areale und zeigt das hohe klinische Potenzial nichtinvasiver Darstellungsmethoden wie DTI in der Diagnostik und Therapie von fokalen Epilepsien.

\subsection{Abschließende Bewertung und Ausblick}

Die vorliegende Studie konnte bei Patienten mit einer Temporallappenepilepsie im Broca-Areal eine atypische funktionelle Sprachlateralisierung im Vergleich zu gesunden Probanden nachweisen, die mit einer atypischen strukturellen Asymmetrie der weißen Substanz in dieser Hirnregion einherging. Für die Wernicke-Region konnten keine signifikanten Unterschiede hinsichtlich der funktionellen Lateralisierung oder der strukturellen Asymmetrie der weißen Substanz zwischen der Patientengruppe und der Kontrollgruppe aufgedeckt werden. Es konnte zwar die Tendenz festgestellt werden, dass auch in dieser Kortexregion die Patienten mit Temporallappenepilepsie ein von gesunden Kontrollprobanden abweichendes funktionelles Aktivierungsmuster sowie eine strukturelle Asymmetrie der weißen Substanz zeigten, diese war jedoch nicht signifikant.

Die Wernicke-Region scheint von pathologischen Prozessen wie einer Temporallappenepilepsie nicht so stark beeinflusst zu werden wie die Broca-Region und daher für die Bestimmung der Sprachlateralisierung nicht so gut geeignet zu sein. 
Eine Ursache dafür könnte eine stärkere Reorganisation von Funktion und Anatomie in der Broca-Region sein. Die fehlende Signifikanz im Wernicke-Areal könnte aber auch ein Effekt der geringen Anzahl der untersuchten Patienten mit fokaler Epilepsie sein. Aufgrund der sehr viel heterogeneren Ergebnisse bei Patienten mit fokaler Epilepsie ist vermutlich eine höhere Anzahl an Untersuchungen nötig, um signifikante pathologische Prozesse zu detektieren. Es bleibt zu prüfen, ob sich aus der Tendenz für die Wernicke-Region bei einem größeren Patientenkollektiv signifikante Unterschiede analog zur Broca-Region ergeben.

Bei der Betrachtung der vorliegenden Ergebnisse muss bedacht werden, dass die untersuchten Probandenkollektive ausschließlich aus Rechtshändern bestanden. Linkshänder wurden in dieser Arbeit nicht untersucht. Die Händigkeit nimmt jedoch großen Einfluss auf die Sprachlateralisierung und kann die Ergebnisse einer Studie stark beeinflussen. Bevor diese Methode als klinische Ergänzung zum Wada-Test eingesetzt werden kann, muss daher gezeigt werden, dass auch bei Linkshändern mit dieser Methode eine verlässliche Aussage über die Lokalisation von Sprachregionen getroffen werden kann.

Darüber hinaus war das Patientenkollektiv hinsichtlich der Pathogenese nicht absolut homogen. Es bestand zwar bei allen in die Studie eingeschlossenen Patienten eine fokale Epilepsie, die Epileptogenese war jedoch nicht komplett einheitlich. Bei zwei Patienten lag der epileptogene Fokus rechtstemporal und bei einer Patientin war die Hemisphäre der Epileptogenese noch unklar. Alle übrigen Patienten wiesen einen linkstemporalen Fokus auf. Bei fünf Patienten war eine linksseitige Hippokampussklerose ursächlich für die Erkrankung und bei einem Patienten bestand eine Hippokampusasymmetrie zuungunsten links. Die übrigen Patienten litten an einer Temporallappenepilepsie entzündlicher oder unklarer Genese (Tabelle 2).

Des Weiteren stellt die BOLD-Antwort nur einen indirekten Hinweis auf die kortikale Aktivität dar. Ein fehlendes BOLD-Signal in Bereichen der grauen Substanz bedeutet nicht zwangsläufig, dass dieser Bereich für kognitive Funktionen bedeutungslos ist. Da in der vorliegenden Studie meist in beiden Hemisphären kortikale Sprachaktivierung durch das Versuchsparadigma induziert wurde, liegt die Vermutung nahe, dass beide Hirnhälften an der Sprachverarbeitung beteiligt sind. Die endgültige Planung von Resektionsgrenzen allein auf Basis der Ergebnisse eines BOLD-Versuches ist daher kritisch zu sehen.

Schließlich wurde im Rahmen dieser Studie kein Wada-Test zur Überprüfung der funktionellen Lateralitätsergebnisse durchgeführt. Aufgrund der Risiken des WadaTests war eine Durchführung bei gesunden Kontrollprobanden oder Patienten ohne Operationsindikation jedoch nicht zu verantworten. Darüber hinaus konnten bereits viele Studien eine bis zu 90\%ige Übereinstimmung des Wada-Tests mit den Ergebnissen funktioneller MRT-Versuche zur Sprachlateralisierung nachweisen (siehe 
Kapitel 4.1 Seite 53), so dass auf diesen invasiven Test verzichtet wurde. Vor einem routinemäßigen klinischen Einsatz ist jedoch als Nachweis der Anwendbarkeit ein Vergleich der hier angewendeten Methode zur Bestimmung der Sprachlateralisierung mit den Ergebnissen eines Wada-Tests unumgänglich.

Es zeigte sich, dass das verwendete Verfahren in dieser Form noch nicht als Routineverfahren für die Praxis geeignet ist, da die Datenverarbeitung und Auswertung sehr aufwendig und nicht automatisierbar sind. Darüber hinaus ist die Messzeit im Scanner sehr lang, so dass eine solche Untersuchung nicht von allen Patienten toleriert wird. Auch bei Kindern oder klaustrophobischen Patienten ist eine Messung im Scanner nicht möglich. Schließlich sind die verwendeten Sprachparadigmen nur bei Patienten anwendbar, deren kognitive Fähigkeiten eine adäquate Durchführung der Sprachtests ermöglichen. Dieses ist jedoch bei Patienten mit neurologischen Erkrankungen nicht immer gewährleistet.

Abschließend kommt die vorliegende Arbeit zu dem Schluss, dass mittels DTIMessungen eine strukturelle Asymmetrie mit höheren FA-Werten in der sprachassoziierten weißen Substanz nachgewiesen werden kann, die gleichsinnig zur funktionellen Sprachlateralisierung besteht. Dieses deutet darauf hin, dass stärkere Aktivität in der grauen Substanz der sprachdominanten Hemisphäre eine höhere Dichte und Gerichtetheit von Leitungsbahnen in der weißen Substanz bedingt, die als strukturelle Veränderungen in der weißen Substanz detektiert werden können. Auch mit DTI-Verfahren ist auf diese Weise die Identifizierung der sprachdominanten Hemisphäre möglich. DTI-Messungen, welche Hinweise auf die Struktur der weißen Substanz liefern, stellen daher eine sinnvolle Ergänzung zu funktionellen MRTParadigmen in der Bestimmung der sprachdominanten Hemisphäre dar. Dennoch zeigte sich, dass in der vorliegenden Arbeit die Gruppe der Patienten mit Epilepsie nicht so eindeutig lateralisierten wie die Kontrollgruppe und daher die Bestimmung der sprachdominanten Hemisphäre für einen epilepsiechirurgischen Eingriff nicht ausreichend sicher erfolgen konnte. Die Ergebnisse könnten jedoch von der Auswahl des Patientenkollektivs beeinträchtigt worden sein. Es bleibt zu prüfen, ob Untersuchungen eines größeren Patientenkollektives mit homogenerer Lokalisation des Fokus sowie eine bessere Selektion der Epileptogenese zu deutlicheren Lateralitäts- und strukturellen Asymmetrieergebnissen führen, welche die in dieser Studie erhobenen Aussagen weiter bestätigen könnten. 


\section{ZUSAMMENFASSUNG}

In der vorliegenden Arbeit wurde durch die Kombination zweier nichtinvasiver MRMethoden in vivo untersucht, ob sich die funktionelle Sprachlateralisierung in einer strukturellen Asymmetrie der weißen Substanz widerspiegelt. Dafür wurden die Ergebnisse einer Patientengruppe mit Temporallappenepilepsie mit den Ergebnissen einer gesunden Kontrollgruppe verglichen. Es konnte gezeigt werden, dass mittels BOLD MRT eine Sprachlateralisierung in der grauen Substanz nachgewiesen werden kann, die mit einem strukturellen Korrelat in der weißen Substanz der sprachdominanten Hemisphäre einhergeht. Dieses Korrelat konnte als Asymmetrie der Mikrostruktur der weißen Substanz in DTI-Messungen belegt werden.

Darüber hinaus konnte bewiesen werden, dass Patienten mit einer Temporallappenepilepsie im Vergleich zu gesunden Probanden eine atypische Lateralisierung der Sprachaktivierung im Broca-Areal aufweisen, die von einer gleichsinnig atypischen strukturelle Asymmetrie der weißen Substanz im Broca-Areal begleitet wird.

Die These, dass eine funktionelle Sprachlateralisierung mit einer mikrostrukturellen Veränderung der weißen Substanz assoziiert ist, wird durch die vorliegende Arbeit sowohl für die gesunden Kontrollprobanden als auch für die Patienten mit einer fokalen Epilepsie bestätigt.

Die Ergebnisse der vorliegenden Arbeit zeigen, dass DTI-Messungen eine sinnvolle Ergänzung zu BOLD MRT-Messungen in der Bestimmung der sprachrelevanten Areale darstellen können. Sprachlateralisierung könnte mit ihrer Hilfe in Zukunft exakt und nichtinvasiv bestimmt werden. Es stellte sich jedoch auch heraus, dass das in der vorliegenden Arbeit angewendete Verfahren in der Praxis sehr aufwendig ist, ohne eine entschieden höhere Aussagekraft über die Sprachlateralisierung zu erbringen. In dieser Form wird die nichtinvasive Bildgebung den Wada-Test als Goldstandard für den klinischen Alltag noch nicht ersetzen können. 


\section{ABKüRZUNGEN}

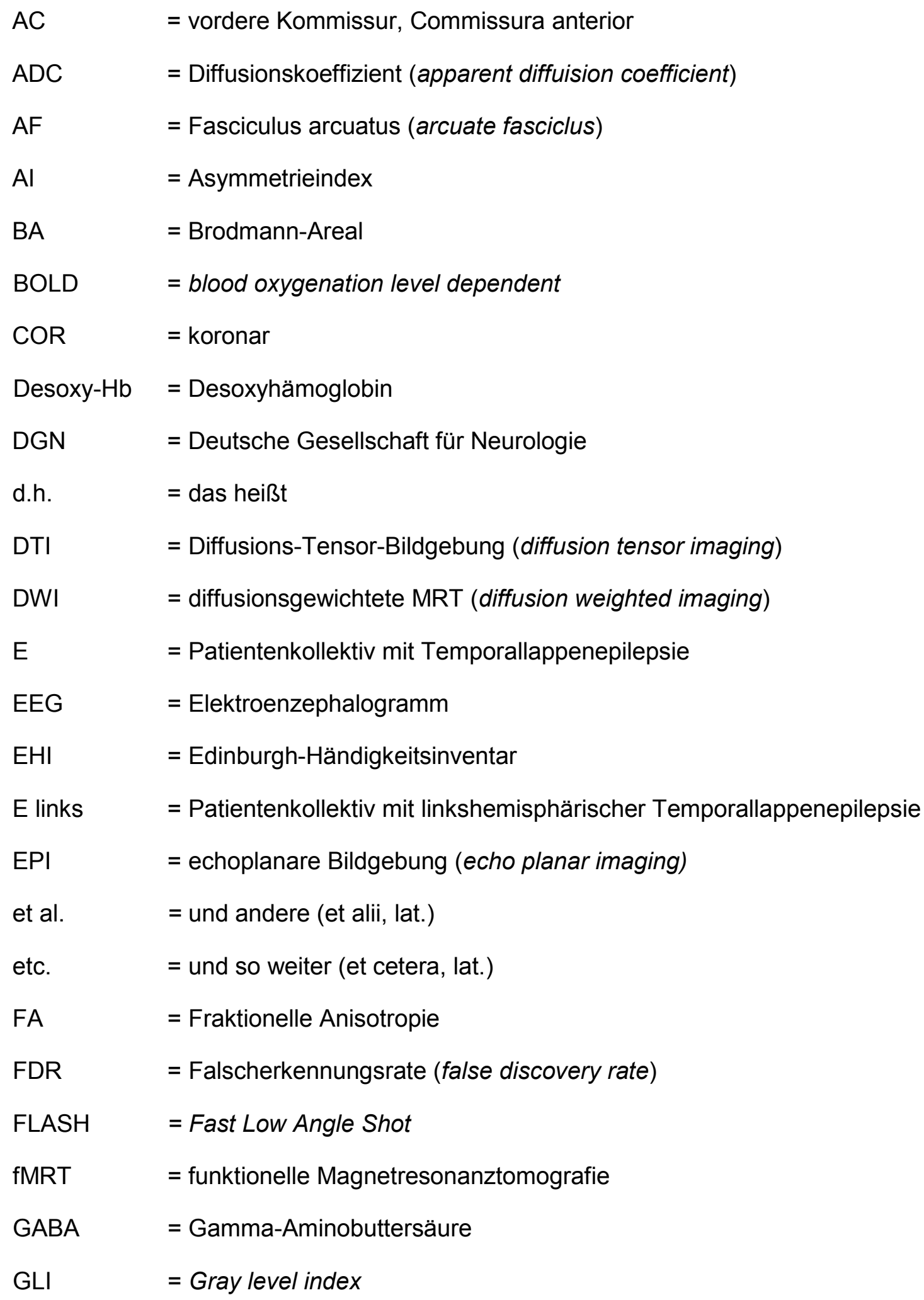




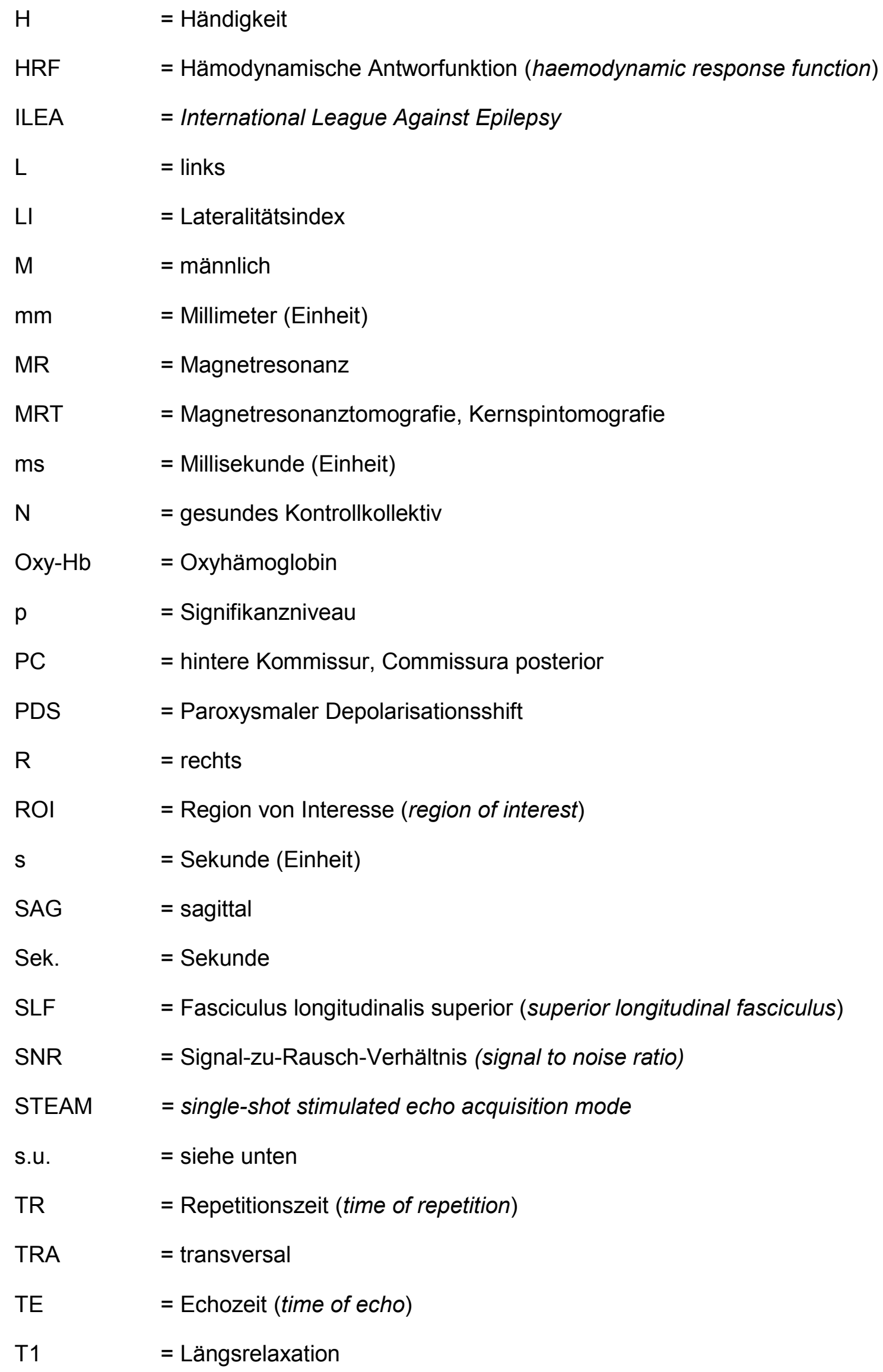


= Querrelaxation

UMG = Universitätsmedizin Göttingen

$\mathrm{W} \quad=$ weiblich

z. B. = zum Beispiel

Z. n. = Zustand nach

ZNS = zentrales Nervensystem 


\section{ANHANG}

\begin{tabular}{|c|c|c|c|c|c|}
\hline Proband & Gruppe & Alter [Jahre] & Geschlecht & $\mathrm{EHI}$ & Händigkeit* \\
\hline 1 & $\mathrm{~N}$ & 24 & M & 100 & $\mathrm{R}$ \\
\hline 2 & $\mathrm{~N}$ & 23 & M & 100 & $\mathrm{R}$ \\
\hline 3 & $\mathrm{~N}$ & 22 & W & 100 & $\mathrm{R}$ \\
\hline 4 & $\mathrm{~N}$ & 23 & M & 100 & $\mathrm{R}$ \\
\hline 5 & $\mathrm{~N}$ & 26 & M & 100 & $\mathrm{R}$ \\
\hline 6 & $\mathrm{~N}$ & 39 & M & 100 & $\mathrm{R}$ \\
\hline 7 & $\mathrm{~N}$ & 37 & M & 100 & $\mathrm{R}$ \\
\hline 8 & $\mathrm{~N}$ & 55 & W & 100 & $\mathrm{R}$ \\
\hline 9 & $\mathrm{~N}$ & 30 & W & 82 & $\mathrm{R}$ \\
\hline 10 & $\mathrm{~N}$ & 42 & M & 100 & $\mathrm{R}$ \\
\hline 11 & $\mathrm{~N}$ & 29 & M & 60 & $R$ \\
\hline 12 & $\mathrm{~N}$ & 28 & M & 100 & $\mathrm{R}$ \\
\hline 13 & $\mathrm{~N}$ & 59 & M & 100 & $\mathrm{R}$ \\
\hline 14 & $\mathrm{~N}$ & 41 & M & 100 & $\mathrm{R}$ \\
\hline 15 & $\mathrm{~N}$ & 38 & M & 87 & $\mathrm{R}$ \\
\hline 16 & $\mathrm{~N}$ & 33 & M & 80 & $\mathrm{R}$ \\
\hline 17 & $\mathrm{~N}$ & 42 & M & 60 & $\mathrm{R}$ \\
\hline 18 & $\mathrm{~N}$ & 33 & M & 100 & $\mathrm{R}$ \\
\hline 19 & $\mathrm{~N}$ & 23 & W & 100 & $\mathrm{R}$ \\
\hline 20 & $\mathrm{~N}$ & 26 & M & 60 & $R$ \\
\hline 21 & $\mathrm{~N}$ & 40 & W & 100 & $\mathrm{R}$ \\
\hline 22 & $\mathrm{~N}$ & 34 & M & 100 & $\mathrm{R}$ \\
\hline 23 & $\mathrm{~N}$ & 31 & W & 70 & $\mathrm{R}$ \\
\hline 24 & $E$ & 21 & $M$ & 100 & $\mathrm{R}$ \\
\hline 25 & E & 45 & M & 80 & $\mathrm{R}$ \\
\hline 26 & E & 41 & M & 80 & $\mathrm{R}$ \\
\hline 27 & E & 31 & M & 100 & $\mathrm{R}$ \\
\hline 28 & E & 51 & w & 100 & $\mathrm{R}$ \\
\hline 29 & $E$ & 28 & M & 100 & $R$ \\
\hline 30 & $E$ & 38 & M & 53 & $\mathrm{R}$ \\
\hline 31 & E & 40 & w & 100 & $R$ \\
\hline 32 & E & 36 & M & 70 & $R$ \\
\hline 33 & E & 57 & w & 100 & $\mathrm{R}$ \\
\hline 34 & $E$ & 39 & M & 100 & $R$ \\
\hline 35 & E & 48 & M & 50 & $\mathrm{R}$ \\
\hline
\end{tabular}




\begin{tabular}{|c|c|c|c|c|c|c|c|c|c|}
\hline $\begin{array}{l}\text { Pro- } \\
\text { band }\end{array}$ & $\begin{array}{l}\text { Klassi- } \\
\text { fikation }\end{array}$ & Ätiologie & Epileptogense & MRT-Befund & EEG-Befund & Medikation & Anfallsart & Anfallsfrequenz & Anmerkungen \\
\hline 24 & $\begin{array}{l}\text { fokale } \\
\text { Epilepsie }\end{array}$ & $\begin{array}{l}\text { Z. n. Variazella- } \\
\text { Zoster- } \\
\text { Enzephalitis }\end{array}$ & $\begin{array}{l}\text { möglicherweise } \\
\text { rechts temporo- } \\
\text { okzipital }\end{array}$ & $\begin{array}{l}\text { Periventrikuläre } \\
\text { Läsionen, mit } \\
\text { postentzündlichen } \\
\text { Veränderungen } \\
\text { vereinbar }\end{array}$ & $\begin{array}{l}\text { interiktales EEG : } \\
\text { rechts temporo- } \\
\text { okzipitaler Herdbefund } \\
\text { ohne epilepsietypische } \\
\text { Muster }\end{array}$ & -Lamotrigin & $\begin{array}{l}\text { einfach-fokal } \\
\text { und sekundär } \\
\text { generalisiert }\end{array}$ & $1 \times$ pro $1 / 2$ Jahr & \\
\hline 25 & $\begin{array}{l}\text { fokale } \\
\text { Epilepsie }\end{array}$ & bisher unbekannt & $\begin{array}{l}\text { möglicherweise } \\
\text { rechts temporal }\end{array}$ & bisher nicht erfolgt & $\begin{array}{l}\text { interiktales EEG: } \\
\text { unauffällig }\end{array}$ & $\begin{array}{l}\text {-Levetiracetam } \\
\text {-Lamotrigin } \\
\text {-Topiramat }\end{array}$ & $\begin{array}{l}\text { komplex-fokal } \\
\text { und sekundär } \\
\text { generalisiert }\end{array}$ & $10 \times$ pro $1 / 2$ Jahr & $\begin{array}{l}\text { - sich } \\
\text { abzeichnende } \\
\text { Pharmako- } \\
\text { resistenz } \\
\text { - ggf. } \\
\text { Epilepsie- } \\
\text { chirurgie }\end{array}$ \\
\hline 26 & $\begin{array}{l}\text { fokale } \\
\text { Epilepsie }\end{array}$ & $\begin{array}{l}\text { Hippokampus- } \\
\text { sklerose links }\end{array}$ & $\begin{array}{l}\text { wahrscheinlich } \\
\text { links temporal }\end{array}$ & $\begin{array}{l}\text { Hippokampus- } \\
\text { sklerose links }\end{array}$ & $\begin{array}{l}\text { interiktales EEG: } \\
\text { Theta intermittierend } \\
\text { regional temporal links }\end{array}$ & $\begin{array}{l}\text {-Lamotrigin } \\
\text {-Levetiracetam } \\
\text {-Pregabalin }\end{array}$ & $\begin{array}{l}\text { komplex-fokal } \\
\text { und sekundär } \\
\text { generalisiert }\end{array}$ & $2 \times$ pro $1 / 2$ Jahr & \\
\hline 27 & $\begin{array}{l}\text { fokale } \\
\text { Epilepsie }\end{array}$ & bisher unbekannt & $\begin{array}{l}\text { wahrscheinlich } \\
\text { links temporal }\end{array}$ & unauffällig & $\begin{array}{l}\text { interiktales EEG: } \\
\text { Theta intermittierend } \\
\text { regional links fronto- } \\
\text { temporal }\end{array}$ & $\begin{array}{l}\text {-Carbamazepin } \\
\text {-Topiramat }\end{array}$ & $\begin{array}{l}\text { einfach-fokal, } \\
\text { komplex-fokal } \\
\text { und sekundär } \\
\text { generalisiert }\end{array}$ & teilweise täglich & $\begin{array}{l}\text { - sich } \\
\text { abzeichnende } \\
\text { Pharmako- } \\
\text { resistenz }\end{array}$ \\
\hline 28 & $\begin{array}{l}\text { fokale } \\
\text { Epilepsie }\end{array}$ & $\begin{array}{l}\text { Hippokampus- } \\
\text { sklerose links }\end{array}$ & $\begin{array}{l}\text { wahrscheinlich } \\
\text { links mesio- } \\
\text { temporal }\end{array}$ & $\begin{array}{l}\text { Hippokampus- } \\
\text { sklerose links }\end{array}$ & $\begin{array}{l}\text { interiktales EEG: } \\
\text { Theta links temporo- } \\
\text { parietal, Theta-Delta- } \\
\text { Aktivität links fronto- } \\
\text { temporal }\end{array}$ & $\begin{array}{l}\text {-Lamotrigin } \\
\text {-Levetiracetam }\end{array}$ & $\begin{array}{l}\text { einfach-fokal, } \\
\text { komplex-fokal } \\
\text { und sekundär } \\
\text { generalisiert }\end{array}$ & $1 \times$ pro Woche & $\begin{array}{l}\text { - zunehmende } \\
\text { Pharmako- } \\
\text { resistenz }\end{array}$ \\
\hline 29 & $\begin{array}{l}\text { fokale } \\
\text { Epilepsie }\end{array}$ & $\begin{array}{l}\text { Hippokampus- } \\
\text { sklerose links }\end{array}$ & $\begin{array}{l}\text { wahrscheinlich } \\
\text { links temporal }\end{array}$ & $\begin{array}{l}\text { Hippokampus- } \\
\text { sklerose links }\end{array}$ & $\begin{array}{l}\text { interiktales EEG: } \\
\text { Theta intermittierend } \\
\text { zentro-temporal links }\end{array}$ & -Lamotrigin & komplex-fokal & $3 \times$ pro Monat & $\begin{array}{l}\text { - Epilepsie- } \\
\text { chirurgie } \\
\text { geplant }\end{array}$ \\
\hline 30 & $\begin{array}{l}\text { fokale } \\
\text { Epilepsie }\end{array}$ & $\begin{array}{l}\text { Hippokampus- } \\
\text { sklerose links }\end{array}$ & $\begin{array}{l}\text { wahrscheinlich } \\
\text { links mesio- } \\
\text { temporal }\end{array}$ & $\begin{array}{l}\text { Hippokampus- } \\
\text { sklerose links }\end{array}$ & $\begin{array}{l}\text { interiktales EEG: } \\
\text { Theta intermittierend bi- } \\
\text { fronto-temporal, } \\
\text { epilepsietypische Muster } \\
\text { links fronto- } \\
\text { temporal/temporo-basal }\end{array}$ & $\begin{array}{l}\text {-Levetiracetam } \\
\text {-Pregabalin } \\
\text {-Mirtazapin }\end{array}$ & $\begin{array}{l}\text { komplex-fokal } \\
\text { und sekundär } \\
\text { generalisiert }\end{array}$ & 3-4 x pro Woche & $\begin{array}{l}\text { - Pharmako- } \\
\text { resistenz } \\
\text { - Epilepsie- } \\
\text { chirurgie } \\
\text { geplant }\end{array}$ \\
\hline
\end{tabular}




\begin{tabular}{|c|c|c|c|c|c|c|c|c|c|}
\hline $\begin{array}{l}\text { Pro- } \\
\text { band }\end{array}$ & $\begin{array}{l}\text { Klassi- } \\
\text { fikation }\end{array}$ & Ätiologie & Epileptogense & MRT-Befund & EEG-Befund & Medikation & Anfallsart & Anfallsfrequenz & Anmerkungen \\
\hline 31 & $\begin{array}{l}\text { fokale } \\
\text { Epilepsie }\end{array}$ & bisher unbekannt & bisher unbekannt & bisher nicht erfolgt & $\begin{array}{l}\text { interiktales EEG: } \\
\text { Theta intermittierend bi- } \\
\text { temporal links betont, } \\
\text { Sharp Waves } \\
\text { intermittierend regional } \\
\text { rechts temporo-basal } \\
\text { und bi-temporal }\end{array}$ & -Lamotrigin & komplex-fokal & $\begin{array}{l}\text { 3-4 } x \text { pro Monat, } \\
\text { seit } 1 \text { Jahr keine } \\
\text { Anfälle mehr }\end{array}$ & \\
\hline 32 & $\begin{array}{l}\text { fokale } \\
\text { Epilepsie }\end{array}$ & bisher unbekannt & $\begin{array}{l}\text { wahrscheinlich } \\
\text { links temporal }\end{array}$ & $\begin{array}{l}\text { postentzündliche } \\
\text { Läsion links } \\
\text { temporal }\end{array}$ & $\begin{array}{l}\text { Schlaf-EEG nach } \\
\text { Schlafentzug: } \\
\text { Theta intermittierend } \\
\text { fronto-temporal links, } \\
\text { Herdbefund links } \\
\text { temporal }\end{array}$ & -Lamotrigin & $\begin{array}{l}\text { sekundär } \\
\text { generalisiert }\end{array}$ & 1x pro Jahr & \\
\hline 33 & $\begin{array}{l}\text { fokale } \\
\text { Epilepsie }\end{array}$ & $\begin{array}{l}\text { Hippokampus- } \\
\text { sklerose links }\end{array}$ & $\begin{array}{l}\text { wahrscheinlich } \\
\text { links temporal }\end{array}$ & $\begin{array}{l}\text { Hippokampus- } \\
\text { sklerose links }\end{array}$ & $\begin{array}{l}\text { interiktales EEG: } \\
\text { Theta intermittierend } \\
\text { fronto-temporal beidseits }\end{array}$ & $\begin{array}{l}\text {-Carbamazepin } \\
\text {-Levetiracetam }\end{array}$ & $\begin{array}{l}\text { komplex-fokal } \\
\text { und sekundär } \\
\text { generalisiert }\end{array}$ & $2 \times$ pro Monat & \\
\hline 34 & $\begin{array}{l}\text { fokale } \\
\text { Epilepsie }\end{array}$ & $\begin{array}{l}\text { Hippokampus- } \\
\text { sklerose links }\end{array}$ & $\begin{array}{l}\text { wahrscheinlich } \\
\text { links mesio- } \\
\text { temporal }\end{array}$ & $\begin{array}{l}\text { Hippokampus- } \\
\text { asymmetrie } \\
\text { zuungunsten links }\end{array}$ & $\begin{array}{l}\text { interiktales EEG: } \\
\text { Theta intermittierend } \\
\text { fronto-temporal rechts }\end{array}$ & -Carbamazepin & $\begin{array}{l}\text { komplex-fokal } \\
\text { und sekundär } \\
\text { generalisiert }\end{array}$ & $\begin{array}{l}\text { mehrere pro } \\
\text { Monat }\end{array}$ & \\
\hline 35 & $\begin{array}{l}\text { fokale } \\
\text { Epilepsie }\end{array}$ & $\begin{array}{l}\text { Z. n. Meningo- } \\
\text { enzaphalitis mit } \\
\text { linkstemporaler } \\
\text { Abszessbildung }\end{array}$ & $\begin{array}{l}\text { wahrscheinlich } \\
\text { links temporal }\end{array}$ & $\begin{array}{l}\text { Abszess links } \\
\text { temporal bei Z. n. } \\
\text { Meningo- } \\
\text { enzephalitis }\end{array}$ & $\begin{array}{l}\text { iktales EEG: } \\
\text { Theta links frontozentral, } \\
\text { z.T. bi-frontotemporal }\end{array}$ & $\begin{array}{l}\text {-Levetiracetam } \\
\text {-Carbamazepin } \\
\text {-Primidon }\end{array}$ & $\begin{array}{l}\text { komplex-fokal } \\
\text { und sekundär } \\
\text { generalisiert }\end{array}$ & $\begin{array}{l}8-13 \times \text { pro } \\
\text { Monat }\end{array}$ & $\begin{array}{l}\text { - ggf. } \\
\text { Epilepsie- } \\
\text { chirurgie }\end{array}$ \\
\hline
\end{tabular}




\begin{tabular}{|c|c|c|c|c|c|c|c|c|}
\hline Proband & $x$ & $\mathrm{Y}$ & z & Seite & Gyrus & BA & Voxel & $p$-Wert \\
\hline 1 & -50.0 & 18.0 & 10.0 & L & Gyrus frontalis inferior & 45 & 5284 & $<0,004379$ \\
\hline 2 & -51.0 & 21.0 & 5.0 & L & Gyrus frontalis inferior & 45 & 14449 & $<0,000532$ \\
\hline 3 & -49.0 & 24.0 & 23.0 & L & Gyrus frontalis medialis & 46 & 5182 & $<0,000002$ \\
\hline 4 & -48.0 & 24.0 & 13.0 & L & Gyrus frontalis inferior & 45 & 7513 & $<0,000001$ \\
\hline 5 & -42.0 & 20.0 & 11.0 & L & Gyrus frontalis inferior & 45 & 4664 & $<0,000001$ \\
\hline 6 & -48.0 & 18.0 & 13.0 & L & Gyrus frontalis inferior & 45 & 5131 & $<0,000205$ \\
\hline 7 & -47.0 & 23.0 & 3.0 & L & Gyrus frontalis inferior & 47 & 4622 & $<0,011844$ \\
\hline 8 & -55.0 & 20.0 & 10.0 & L & Gyrus frontalis inferior & 45 & 2300 & $<0,001274$ \\
\hline 9 & -34.0 & 16.0 & 15.0 & L & Insel & 13 & 9456 & $<0,047192$ \\
\hline 10 & -39.0 & 18.0 & 7.0 & L & Insel & 13 & 8914 & $<0,000097$ \\
\hline 11 & -39.0 & 25.0 & 11.0 & L & Gyrus frontalis inferior & 13 & 5726 & $<0,000851$ \\
\hline 12 & -48.0 & 17.0 & 16.0 & L & Gyrus frontalis inferior & 45 & 5055 & $<1,2738 \cdot 10^{-08}$ \\
\hline 13 & -51.0 & 15.0 & 4.0 & L & Gyrus frontalis inferior & 45 & 1663 & $<1,2738 \cdot 10^{-08}$ \\
\hline 14 & -48.0 & 18.0 & 25.0 & L & Gyrus frontalis inferior & 9 & 6031 & $<0,000020$ \\
\hline 15 & -46.0 & 12.0 & 17.0 & L & Gyrus frontalis inferior & 44 & 3830 & $<0,000001$ \\
\hline 16 & -48.0 & 13.0 & 15.0 & L & Gyrus frontalis inferior & 44 & 6328 & $<0,047192$ \\
\hline 17 & -36.0 & 23.0 & 15.0 & L & Insel & 13 & 3850 & $<0,000424$ \\
\hline 18 & & & & & & & 0 & \\
\hline 19 & -44.0 & 23.0 & 6.0 & L & Gyrus frontalis inferior & 13 & 3564 & $<6,6637 \cdot 10^{-11}$ \\
\hline 20 & -39.0 & 18.0 & 11.0 & L & Insel & 13 & 5954 & 0,000097 \\
\hline 21 & -40.0 & 18.0 & 15.0 & L & Gyrus frontalis inferior & 45 & 12328 & $<0,003132$ \\
\hline 22 & -40.0 & 26.0 & 17.0 & L & Gyrus frontalis medialis & 46 & 4652 & $<5,739 \cdot 10^{-10}$ \\
\hline 23 & -32.0 & 29.0 & 10.0 & L & Gyrus frontalis inferior & 45 & 3073 & $<0,000097$ \\
\hline
\end{tabular}




\begin{tabular}{|c|c|c|c|c|c|c|c|c|}
\hline Proband & $x$ & Y & Z & Seite & Gyrus & BA & Voxel & $p$ \\
\hline 1 & 49.0 & 21.0 & 20.0 & $\mathrm{R}$ & Gyrus frontalis medialis & 46 & 2015 & $<0,004379$ \\
\hline 2 & 43.0 & 14.0 & 5.0 & $R$ & Insel & 13 & 4055 & $<0,000532$ \\
\hline 3 & 49.0 & 19.0 & 4.0 & $R$ & Gyrus frontalis inferior & 45 & 326 & $<0,000002$ \\
\hline 4 & & & & & & & 0 & $<0,000001$ \\
\hline 5 & & & & & & & 0 & $<0,000001$ \\
\hline 6 & & & & & & & 0 & $<0,000205$ \\
\hline 7 & 51.0 & 21.0 & 2.0 & $R$ & Gyrus frontalis inferior & 47 & 802 & $<0,011844$ \\
\hline 8 & 42.0 & 17.0 & 5.0 & $R$ & Insel & 13 & 4733 & $<0,001274$ \\
\hline 9 & 45.0 & 16.0 & 10.0 & $R$ & Gyrus frontalis inferior & 44 & 7040 & $<0,047192$ \\
\hline 10 & 41.0 & 18.0 & 5.0 & $R$ & Insel & 13 & 3221 & $<0,000097$ \\
\hline 11 & 36.0 & 21.0 & 9.0 & $R$ & Gyrus frontalis inferior & 13 & 3146 & $<0,000851$ \\
\hline 12 & & & & & & & 0 & $<1,2738 \cdot 10^{-08}$ \\
\hline 13 & & & & & & & 0 & $<1,2738 \cdot 10^{-08}$ \\
\hline 14 & 33.0 & 18.0 & 15.0 & $R$ & Insel & 13 & 2747 & $<0,000020$ \\
\hline 15 & & & & & & & 0 & $<0,000001$ \\
\hline 16 & & & & & & & 0 & $<0,047192$ \\
\hline 17 & 40.0 & 25.0 & 14.0 & $R$ & Insel & 13 & 5074 & $<0,000424$ \\
\hline 18 & & & & & & & 0 & \\
\hline 19 & & & & & & & 0 & $<6,6637 \cdot 10^{-11}$ \\
\hline 20 & 43.0 & 15.0 & 6.0 & $R$ & Gyrus praecentralis & 44 & 2290 & 0,000097 \\
\hline 21 & 38.0 & 17.0 & 13.0 & $R$ & Insel & 13 & 2155 & $<0,003132$ \\
\hline 22 & 40.0 & 18.0 & 10.0 & $R$ & Insel & 13 & 124 & $<5,739 \cdot 10^{-10}$ \\
\hline 23 & & & & & & & 0 & $<0,000097$ \\
\hline
\end{tabular}




\begin{tabular}{|c|c|c|c|c|c|c|c|c|}
\hline Proband & $x$ & $\mathrm{Y}$ & Z & Seite & Gyrus & BA & Voxel & $p$-Wert \\
\hline 24 & -39.0 & 15.0 & 10.0 & L & Insel & 13 & 9888 & $<0,005740$ \\
\hline 25 & -42.0 & 2.0 & 36.0 & L & Gyrus praecentralis & 6 & 5069 & $<0,017544$ \\
\hline 26 & -48.0 & 19.0 & 15.0 & L & Gyrus frontalis inferior & 45 & 5255 & $<0,000097$ \\
\hline 27 & -48.0 & 7.0 & 12.0 & $L$ & Gyrus praecentralis & 44 & 14118 & $<0,000009$ \\
\hline 28 & -42.0 & 25.0 & 12.0 & L & Gyrus frontalis inferior & 13 & 16986 & $<0,003132$ \\
\hline 29 & -42.0 & 18.0 & 11.0 & $L$ & Gyrus frontalis inferior & 45 & 11848 & $<0,047192$ \\
\hline 30 & -40.0 & 9.0 & 13.0 & $L$ & Insel & 13 & 6578 & $<0,017544$ \\
\hline 31 & -41.0 & 16.0 & 19.0 & L & Gyrus frontalis medialis & 46 & 9090 & $<0,047192$ \\
\hline 32 & -32.0 & 21.0 & 4.0 & L & Insel & 13 & 7917 & $<0,010195$ \\
\hline 33 & -35.0 & 13.0 & 7.0 & L & Insel & 13 & 3592 & $<0,010195$ \\
\hline 34 & -38.0 & 19.0 & 9.0 & L & Insel & 13 & 9144 & $<0,047192$ \\
\hline 35 & -46.0 & 18.0 & 4.0 & L & Gyrus frontalis inferior & 45 & 561 & $<0,010195$ \\
\hline
\end{tabular}

\begin{tabular}{|c|c|c|c|c|c|c|c|c|}
\hline Proband & $x$ & $\mathrm{Y}$ & $Z$ & Seite & Gyrus & BA & Voxel & $p$ \\
\hline 24 & 37.0 & 16.0 & 14.0 & $R$ & Insel & 13 & 6553 & $<0,005740$ \\
\hline 25 & 49.0 & 8.0 & 19.0 & $R$ & Gyrus frontalis inferior & 44 & 570 & $<0,017544$ \\
\hline 26 & 51.0 & 24.0 & 14.0 & $R$ & Gyrus frontalis inferior & 45 & 6935 & $<0,000097$ \\
\hline 27 & 31.0 & 9.0 & 9.0 & $R$ & Claustrum & * & 10137 & $<0,000009$ \\
\hline 28 & 43.0 & 37.0 & 18.0 & $R$ & Gyrus frontalis medialis & 46 & 2278 & $<0,003132$ \\
\hline 29 & 35.0 & 15.0 & 11.0 & $R$ & Insel & 13 & 3812 & $<0,047192$ \\
\hline 30 & 40.0 & 13.0 & 7.0 & $R$ & Insel & 13 & 9251 & $<0,017544$ \\
\hline 31 & & & & & & & 0 & $<0,047192$ \\
\hline 32 & 31.0 & 20.0 & 3.0 & $R$ & Insel & * & 2964 & $<0,010195$ \\
\hline 33 & 50.0 & 14.0 & 13.0 & $R$ & Gyrus frontalis inferior & 44 & 3546 & $<0,010195$ \\
\hline 34 & 32.0 & 26.0 & 5.0 & $\mathrm{R}$ & Gyrus frontalis inferior & 45 & 1130 & $<0,047192$ \\
\hline 35 & 34.0 & 24.0 & 0.0 & $\mathrm{R}$ & Gyrus frontalis inferior & 47 & 4116 & $<0,010195$ \\
\hline \multicolumn{9}{|c|}{$\begin{array}{l}\text { Wichtige Informationen über die ausgewertete ROI im Gebiet der Broca-Region der rechten Hemisphäre der fMRT-Daten: } \\
\text { Talairachkoordinaten des Aktivitätsschwerpunktes, Seitenlokalisation der Aktivierung, anatomische Lage und zugehöriges } \\
\text { BA des Aktivitätsschwerpunktes, Anzahl der signifikant aktivierten Voxel, statistische Schwelle }\end{array}$} \\
\hline \multicolumn{9}{|c|}{ * Aktivitätsschwerpunkt konnte keinem BA zugeordnet werden } \\
\hline
\end{tabular}




\begin{tabular}{|c|c|c|c|c|c|}
\hline Proband & Gruppe & Voxel links & Voxel rechts & LI ROI „Wort Broca“ & Lateralisierung \\
\hline 1 & $\mathrm{~N}$ & 5284 & 2015 & 0,447869571 & L \\
\hline 2 & $\mathrm{~N}$ & 14449 & 4055 & 0,561716386 & L \\
\hline 3 & $\mathrm{~N}$ & 5182 & 326 & 0,881626725 & L \\
\hline 4 & $\mathrm{~N}$ & 7513 & 0 & 1 & L \\
\hline 5 & $\mathrm{~N}$ & 4664 & 0 & 1 & L \\
\hline 6 & $\mathrm{~N}$ & 5131 & 0 & 1 & L \\
\hline 7 & $\mathrm{~N}$ & 4622 & 802 & 0,704277286 & L \\
\hline 8 & $\mathrm{~N}$ & 2300 & 4733 & $-0,345940566$ & $\mathrm{R}$ \\
\hline 9 & $\mathrm{~N}$ & 9456 & 7040 & 0,146459748 & L \\
\hline 10 & $\mathrm{~N}$ & 8914 & 3221 & 0,469138855 & L \\
\hline 11 & $\mathrm{~N}$ & 5726 & 3146 & 0,290802525 & L \\
\hline 12 & $\mathrm{~N}$ & 5055 & 0 & 1 & L \\
\hline 13 & $\mathrm{~N}$ & 1663 & 0 & 1 & L \\
\hline 14 & $\mathrm{~N}$ & 6031 & 2747 & 0,374117111 & L \\
\hline 15 & $\mathrm{~N}$ & 3830 & 0 & 1 & L \\
\hline 16 & $\mathrm{~N}$ & 6328 & 0 & 1 & L \\
\hline 17 & $\mathrm{~N}$ & 3850 & 5074 & $-0,137158225$ & $\mathrm{R}$ \\
\hline 18 & $\mathrm{~N}$ & 0 & 0 & & \\
\hline 19 & $\mathrm{~N}$ & 3564 & 0 & 1 & L \\
\hline 20 & $\mathrm{~N}$ & 5954 & 2290 & 0,444444444 & L \\
\hline 21 & $\mathrm{~N}$ & 12328 & 2155 & 0,702409722 & L \\
\hline 22 & $N$ & 4652 & 124 & 0,948073702 & L \\
\hline 23 & $\mathrm{~N}$ & 3073 & 0 & 1 & L \\
\hline 24 & $\mathrm{E}$ & 9888 & 6553 & 0,202846542 & L \\
\hline 25 & $\mathrm{E}$ & 5069 & 570 & 0,797836496 & L \\
\hline 26 & E & 5255 & 6935 & $-0,137817884$ & $\mathrm{R}$ \\
\hline 27 & $E$ & 14118 & 10137 & 0,164131107 & L \\
\hline 28 & E & 16986 & 2278 & 0,763496678 & L \\
\hline 29 & E & 11848 & 3812 & 0,513154534 & L \\
\hline 30 & $\mathrm{E}$ & 6578 & 9251 & $-0,168867269$ & $\mathrm{R}$ \\
\hline 31 & E & 9090 & 0 & 1 & L \\
\hline 32 & $\mathrm{E}$ & 7917 & 2964 & 0,455197133 & L \\
\hline 33 & E & 3592 & 3546 & 0,006444382 & $\mathrm{~S}$ \\
\hline 34 & E & 9144 & 1130 & 0,780027253 & L \\
\hline 35 & $\mathrm{E}$ & 561 & 4116 & $-0,76010263$ & $R$ \\
\hline
\end{tabular}




\begin{tabular}{|c|c|c|c|c|c|c|c|c|}
\hline Proband & $x$ & $\mathrm{Y}$ & $z$ & Seite & Gyrus & BA & Voxel & $p$-Wert \\
\hline 1 & -59.0 & -41.0 & 13.0 & $\mathrm{~L}$ & Gyrus temporalis superior & 22 & 1816 & $<0,004379$ \\
\hline 2 & -61.0 & -17.0 & 0.0 & L & Gyrus temporalis superior & 22 & 3517 & $<0,000532$ \\
\hline 3 & -53.0 & -35.0 & 7.0 & L & Gyrus temporalis medialis & 22 & 4135 & $<0,000002$ \\
\hline 4 & -54.0 & -37.0 & 11.0 & L & Gyrus temporalis superior & 22 & 1964 & $<0,000001$ \\
\hline 5 & -59.0 & -38.0 & 12.0 & L & Gyrus temporalis superior & 22 & 6716 & $<0,000001$ \\
\hline 6 & -57.0 & -27.0 & 0.0 & L & Gyrus temporalis superior & * & 476 & $<0,000205$ \\
\hline 7 & -56.0 & -34.0 & 19.0 & L & Insel & & 1885 & $<0,011844$ \\
\hline 8 & & & & & & & 0 & $<0,001274$ \\
\hline 9 & -52.0 & -30.0 & 13.0 & L & Gyrus temporalis superior & 41 & 842 & $<0,047192$ \\
\hline 10 & -58.0 & -41.0 & 4.0 & L & Gyrus temporalis medialis & 22 & 5395 & $<0,000097$ \\
\hline 11 & -55.0 & -30.0 & 1.0 & L & Gyrus temporalis medialis & 21 & 3046 & $<0,000851$ \\
\hline 12 & -53.0 & -32.0 & 5.0 & L & Gyrus temporalis medialis & 22 & 1725 & $<1,2738 \cdot 10^{-08}$ \\
\hline 13 & -60.0 & -34.0 & 3.0 & L & Gyrus temporalis medialis & 22 & 2468 & $<1,2738 \cdot 10^{-08}$ \\
\hline 14 & -53.0 & -43.0 & -2.0 & L & Gyrus temporalis medialis & 22 & 598 & $<0,000020$ \\
\hline 15 & -61.0 & -49.0 & 14.0 & L & ** & & 709 & $<0,000001$ \\
\hline 16 & -55.0 & -10.0 & -8.0 & L & Gyrus temporalis medialis & 21 & 1770 & $<0,047192$ \\
\hline 17 & & & & & & & 0 & $<0,000424$ \\
\hline 18 & -64.0 & -26.0 & 12.0 & L & Gyrus temporalis superior & 42 & 575 & $<0,003132$ \\
\hline 19 & -48.0 & -43.0 & 5.0 & L & Gyrus temporalis medialis & 22 & 757 & $<6,6637 \cdot 10^{-11}$ \\
\hline 20 & -54.0 & -33.0 & 13.0 & L & Gyrus temporalis superior & 42 & 991 & 0,000097 \\
\hline 21 & -64.0 & -23.0 & 9.0 & L & Gyrus temporalis superior & 42 & 418 & $<0,003132$ \\
\hline 22 & -59.0 & -26.0 & 9.0 & L & Gyrus temporalis superior & 41 & 640 & $<5,739 \cdot 10^{-10}$ \\
\hline 23 & -65.0 & -27.0 & 6.0 & L & Gyrus temporalis superior & 42 & 240 & $<0,000097$ \\
\hline \multicolumn{9}{|c|}{$\begin{array}{l}\text { Wichtige Informationen über die ausgewertete Region of Interest im Gebiet der Wernicke-Region der linken Hemisphäre de } \\
\text { funktionellen MRT-Daten: Talairachkoordinaten des Aktivitätsschwerpunktes, Seitenlokalisation der Aktivierung, } \\
\text { anatomische Lage und zugehöriges BA des Aktivitätsschwerpunktes, Anzahl der signifikant aktivierten Voxel, statistische } \\
\text { Schwelle } \\
\text { * Aktivitätsschwerpunkt konnte keinem BA zugeordnet werden; }{ }^{* *} \text { Aktivitätsschwerpunkt konnte keine graue Substanz } \\
\text { zugeordnet werden }\end{array}$} \\
\hline
\end{tabular}




\begin{tabular}{|c|c|c|c|c|c|c|c|c|}
\hline Proband & $x$ & $\mathrm{Y}$ & Z & Seite & Gyrus & BA & Voxel & $p$-Wert \\
\hline 1 & 47.0 & 9.0 & -35.0 & $R$ & Gyrus temporalis medialis & 21 & 6034 & $<0,004379$ \\
\hline 2 & 59.0 & -30.0 & 3.0 & $R$ & Gyrus temporalis medialis & 22 & 3570 & $<0,000532$ \\
\hline 3 & 51.0 & -31.0 & 4.0 & $R$ & Gyrus temporalis superior & 22 & 5088 & $<0,000002$ \\
\hline 4 & & & & & & & 0 & $<0,000001$ \\
\hline 5 & 56.0 & -35.0 & 6.0 & $R$ & Gyrus temporalis medialis & 22 & 1400 & $<0,000001$ \\
\hline 6 & & & & & & & 0 & $<0,000205$ \\
\hline 7 & 63.0 & -34.0 & 16.0 & $R$ & Gyrus temporalis superior & 42 & 1121 & $<0,011844$ \\
\hline 8 & 48.0 & -26.0 & -2.0 & $R$ & Gyrus temporalis superior & 21 & 840 & $<0,001274$ \\
\hline 9 & 51.0 & -27.0 & 4.0 & $R$ & Gyrus temporalis superior & 22 & 1099 & $<0,047192$ \\
\hline 10 & 49.0 & -32.0 & 10.0 & $R$ & Gyrus temporalis superior & 41 & 1690 & $<0,000097$ \\
\hline 11 & 51.0 & -30.0 & 4.0 & $R$ & Gyrus temporalis superior & 22 & 1231 & $<0,000851$ \\
\hline 12 & & & & & & & 0 & $<1,2738 \cdot 10^{-08}$ \\
\hline 13 & & & & & & & 0 & $<1,2738 \cdot 10^{-08}$ \\
\hline 14 & & & & & & & 0 & $<0,000020$ \\
\hline 15 & & & & & & & 0 & $<0,000001$ \\
\hline 16 & & & & & & & 0 & $<0,047192$ \\
\hline 17 & 49.0 & -23.0 & -5.0 & $R$ & Gyrus temporalis superior & 21 & 858 & $<0,000424$ \\
\hline 18 & 46.0 & -25.0 & 10.0 & $R$ & Gyrus temporalis superior & 41 & 388 & $<0,003132$ \\
\hline 19 & & & & & & & 0 & $<6,6637 \cdot 10^{-11}$ \\
\hline 20 & 48.0 & -25.0 & 0.0 & $R$ & Gyrus temporalis superior & 21 & 1302 & 0,000097 \\
\hline 21 & & & & & & & 0 & $<0,003132$ \\
\hline 22 & & & & & & & 0 & $<5,739 \cdot 10^{-10}$ \\
\hline 23 & 49.0 & -31.0 & 3.0 & $R$ & Gyrus temporalis superior & 22 & 27 & $<0,000097$ \\
\hline \multicolumn{9}{|c|}{$\begin{array}{l}\text { Wichtige Informationen über die ausgewertete Region of Interest im Gebiet der Wernicke-Region der rechten Hemisphäre } \\
\text { der funktionellen MRT-Daten: Talairachkoordinaten des Aktivitätsschwerpunktes, Seitenlokalisation der Aktivierung, } \\
\text { anatomische Lage und zugehöriges BA des Aktivitätsschwerpunktes, Anzahl der signifikant aktivierten Voxel, statistische } \\
\text { Schwelle }\end{array}$} \\
\hline
\end{tabular}




\begin{tabular}{|c|c|c|c|c|c|c|c|c|}
\hline Proband & $x$ & Y & Z & Seite & Gyrus & BA & Voxel & $p$-Wert \\
\hline 24 & -48.0 & -33.0 & 6.0 & L & Gyrus temporalis superior & 22 & 1012 & $<0,005740$ \\
\hline 25 & -62.0 & -41.0 & 6.0 & L & Gyrus temporalis medialis & 22 & 1560 & $<0,017544$ \\
\hline 26 & -53.0 & -40.0 & 2.0 & L & Gyrus temporalis medialis & 22 & 4518 & $<0,000097$ \\
\hline 27 & -53.0 & -40.0 & -5.0 & L & subgyral & 37 & 3334 & $<0,000009$ \\
\hline 28 & -57.0 & -31.0 & 9.0 & L & Gyrus temporalis superior & 42 & 5965 & $<0,003132$ \\
\hline 29 & -50.0 & -41.0 & 6.0 & L & Gyrus temporalis medialis & 22 & 275 & $<0,047192$ \\
\hline 30 & -49.0 & -38.0 & 8.0 & L & Gyrus temporalis superior & 22 & 512 & $<0,017544$ \\
\hline 31 & -47.0 & -46.0 & 15.0 & L & Gyrus temporalis superior & 13 & 765 & $<0,047192$ \\
\hline 32 & -51.0 & -25.0 & 9.0 & L & Gyrus temporalis superior & 41 & 1785 & $<0,010195$ \\
\hline 33 & -47.0 & -41.0 & 8.0 & L & Gyrus temporalis medialis & 22 & 1974 & $<0,010195$ \\
\hline 34 & -48.0 & -37.0 & -4.0 & L & Gyrus temporalis medialis & * & 2825 & $<0,047192$ \\
\hline 35 & -57.0 & -40.0 & -7.0 & L & Gyrus temporalis medialis & 20 & 1373 & $<0,010195$ \\
\hline \multicolumn{9}{|c|}{$\begin{array}{l}\text { Wichtige Informationen über die ausgewertete ROI im Gebiet der Wernicke-Region der linken Hemisphäre der funktionellen } \\
\text { MRT-Daten: Talairachkoordinaten des Aktivitätsschwerpunktes, Seitenlokalisation der Aktivierung, anatomische Lage und } \\
\text { zugehöriges BA des Aktivitätsschwerpunktes, Anzahl der signifikant aktivierten Voxel, statistische Schwelle } \\
\text { * Aktivitätsschwerpunkt konnte keinem BA zugeordnet werden }\end{array}$} \\
\hline
\end{tabular}

\begin{tabular}{|l|l|l|l|l|l|l|l|l|}
\hline \multicolumn{7}{|l|}{ Tabelle 11: Lage der Aktivitätsschwerpunkte der ROI „Wort Wernicke“ der rechten Hemisphäre; Gruppe E } \\
\hline Proband & $\mathrm{X}$ & $\mathrm{Y}$ & $\mathrm{Z}$ & Seite & Gyrus & BA & Voxel & $\mathrm{p}$-Wert \\
\hline 24 & 49.0 & -23.0 & 2.0 & $\mathrm{R}$ & Gyrus temporalis superior & 22 & 2494 & $<0,005740$ \\
25 & 52.0 & -36.0 & 3.0 & $\mathrm{R}$ & Gyrus temporalis medialis & 22 & 2688 & $<0,017544$ \\
26 & 49.0 & -41.0 & 4.0 & $\mathrm{R}$ & Gyrus temporalis medialis & 22 & 1639 & $<0,000097$ \\
27 & & & & & & & 0 & $<0,000009$ \\
28 & & & & & & & & $<0,003132$ \\
29 & 44.0 & -37.0 & 10.0 & $\mathrm{R}$ & Gyrus temporalis superior & 41 & 157 & $<0,017544$ \\
30 & 52.0 & -31.0 & 13.0 & $\mathrm{R}$ & Gyrus temporalis superior & 41 & 605 & $<0,047192$ \\
31 & 45.0 & -29.0 & 3.0 & $\mathrm{R}$ & Gyrus temporalis superior & 41 & 381 & $<0,010195$ \\
32 & 52.0 & -25.0 & 4.0 & $\mathrm{R}$ & Gyrus temporalis superior & 22 & 844 & $<0,010195$ \\
33 & 50.0 & -45.0 & 21.0 & $\mathrm{R}$ & Gyrus temporalis superior & 13 & 574 & $<0,047192$ \\
34 & 62.0 & -25.0 & 0.0 & $\mathrm{R}$ & Gyrus temporalis superior & 22 & 2289 & $<0,010195$ \\
35 &
\end{tabular}




\begin{tabular}{|c|c|c|c|c|c|}
\hline Proband & Gruppe & Voxel links & Voxel rechts & LI „Wort Wernicke“ & Lateralisierung \\
\hline 1 & $\mathrm{~N}$ & 1816 & 6034 & $-0,537324841$ & $R$ \\
\hline 2 & $\mathrm{~N}$ & 3517 & 3570 & $-0,007478482$ & $S$ \\
\hline 3 & $\mathrm{~N}$ & 4135 & 5088 & $-0,103328635$ & $R$ \\
\hline 4 & $\mathrm{~N}$ & 1964 & 0 & 1 & L \\
\hline 5 & $\mathrm{~N}$ & 6716 & 1400 & 0,655002464 & L \\
\hline 6 & $\mathrm{~N}$ & 476 & 0 & 1 & L \\
\hline 7 & $\mathrm{~N}$ & 1885 & 1121 & 0,25415835 & L \\
\hline 8 & $\mathrm{~N}$ & 0 & 840 & -1 & $R$ \\
\hline 9 & $\mathrm{~N}$ & 842 & 1099 & $\mid-0,132405976$ & $\mathrm{R}$ \\
\hline 10 & N & 5395 & 1690 & 0,52293578 & L \\
\hline 11 & N & 3046 & 1231 & 0,424362871 & L \\
\hline 12 & $\mathrm{~N}$ & 1725 & 0 & 1 & L \\
\hline 13 & N & 2468 & 0 & 1 & L \\
\hline 14 & N & 598 & 0 & 1 & L \\
\hline 15 & $\mathrm{~N}$ & 709 & 0 & 1 & L \\
\hline 16 & N & 1770 & 0 & 1 & L \\
\hline 17 & N & 0 & 858 & -1 & $R$ \\
\hline 18 & N & 575 & 388 & 0,194184839 & L \\
\hline 19 & N & 757 & 0 & 1 & L \\
\hline 20 & N & 991 & 1302 & $-0,135630179$ & $R$ \\
\hline 21 & N & 418 & 0 & 1 & L \\
\hline 22 & N & 640 & 0 & 1 & L \\
\hline 23 & N & 240 & 27 & 0,797752809 & L \\
\hline 24 & $E$ & 1012 & 2494 & $-0,422703936$ & $R$ \\
\hline 25 & $E$ & 1560 & 2688 & $-0,265536723$ & $R$ \\
\hline 26 & $E$ & 4518 & 1639 & 0,467597856 & L \\
\hline 27 & $E$ & 3334 & 0 & 1 & L \\
\hline 28 & $E$ & 5965 & 0 & 1 & L \\
\hline 29 & $E$ & 275 & 0 & 1 & L \\
\hline 30 & E & 512 & 157 & 0,53064275 & L \\
\hline 31 & $E$ & 765 & 605 & 0,116788321 & L \\
\hline 32 & $E$ & 1785 & 381 & 0,648199446 & L \\
\hline 33 & $E$ & 1974 & 844 & 0,400993612 & L \\
\hline 34 & $E$ & 2825 & 574 & 0,662253604 & L \\
\hline 35 & E & 1373 & 2289 & $\mid-0,250136537$ & $R$ \\
\hline
\end{tabular}




\begin{tabular}{|c|c|c|c|c|c|c|c|c|}
\hline Proband & $x$ & $\mathrm{Y}$ & $z$ & Seite & Gyrus & BA & Voxel & $p$-Wert \\
\hline 1 & -50.0 & 23.0 & 11.0 & L & Gyrus frontalis inferior & 45 & 6316 & $<0,000168$ \\
\hline 2 & -50.0 & 23.0 & 7.0 & L & Gyrus frontalis inferior & 45 & 12508 & $<0,000066$ \\
\hline 3 & -52.0 & 18.0 & 22.0 & L & Gyrus frontalis inferior & 9 & 6694 & $<0,000066$ \\
\hline 4 & -48.0 & 25.0 & 12.0 & L & Gyrus frontalis inferior & 45 & 6566 & $<0,000097$ \\
\hline 5 & -43.0 & 21.0 & 8.0 & L & Gyrus frontalis inferior & 13 & 3769 & $<0,000001$ \\
\hline 6 & -50.0 & 22.0 & 11.0 & L & Gyrus frontalis inferior & 45 & 8032 & $<1,2738 \cdot 10^{-08}$ \\
\hline 7 & -45.0 & 14.0 & 2.0 & L & Insel & 13 & 987 & $<0,022423$ \\
\hline 8 & -48.0 & 26.0 & 8.0 & L & Gyrus frontalis inferior & 45 & 5197 & $<0,000151$ \\
\hline 9 & -34.0 & 24.0 & 13.0 & L & Insel & 13 & 2514 & $<0,047192$ \\
\hline 10 & -48.0 & 20.0 & 16.0 & L & Gyrus frontalis inferior & 45 & 6701 & $<0,000097$ \\
\hline 11 & -52.0 & 26.0 & 14.0 & L & Gyrus frontalis inferior & 46 & 7725 & $<0,000097$ \\
\hline 12 & -49.0 & 23.0 & 5.0 & L & Gyrus frontalis inferior & 45 & 2439 & $<6,6637 \cdot 10^{-11}$ \\
\hline 13 & -54.0 & 16.0 & 6.0 & L & Gyrus praecentralis & 44 & 1956 & $<6,6637 \cdot 10^{-11}$ \\
\hline 14 & -47.0 & 23.0 & 18.0 & L & Gyrus frontalis inferior & 45 & 3034 & $<0,000001$ \\
\hline 15 & -47.0 & 12.0 & 16.0 & L & Gyrus frontalis inferior & 44 & 5633 & $<6,6637 \cdot 10^{-11}$ \\
\hline 16 & -42.0 & 21.0 & 13.0 & L & Gyrus frontalis inferior & 45 & 7864 & $<0,000044$ \\
\hline 17 & -49.0 & 22.0 & 3.0 & L & Gyrus frontalis inferior & 47 & 430 & $<0,000424$ \\
\hline \multicolumn{9}{|l|}{18} \\
\hline 19 & -45.0 & 21.0 & 6.0 & L & Gyrus frontalis inferior & 45 & 3460 & $<6,6637 \cdot 10^{-11}$ \\
\hline 20 & -42.0 & 19.0 & 10.0 & L & Gyrus frontalis inferior & 45 & 6418 & 0,000001 \\
\hline 21 & -40.0 & 21.0 & 7.0 & L & Gyrus frontalis inferior & 13 & 10941 & $<0,003132$ \\
\hline 22 & -42.0 & 25.0 & 17.0 & L & Gyrus frontalis medialis & 46 & 5624 & $<7,5117 \cdot 10^{-13}$ \\
\hline 23 & -34.0 & 27.0 & 10.0 & L & Gyrus frontalis inferior & 13 & 3642 & $<0,003132$ \\
\hline
\end{tabular}




\begin{tabular}{|c|c|c|c|c|c|c|c|c|}
\hline Proband & $x$ & Y & Z & Seite & Gyrus & BA & Voxel & $p$-Wert \\
\hline 24 & -44.0 & 23.0 & 7.0 & L & Gyrus frontalis inferior & 13 & 2374 & $<0,005740$ \\
\hline 25 & -45.0 & 8.0 & 23.0 & L & Gyrus frontalis inferior & 9 & 5043 & $<0,003132$ \\
\hline 26 & -44.0 & 17.0 & 15.0 & L & Gyrus frontalis inferior & 45 & 6441 & $<0,000424$ \\
\hline 27 & -48.0 & 13.0 & 13.0 & L & Gyrus frontalis inferior & 44 & 12898 & $<0,000020$ \\
\hline 28 & -41.0 & 29.0 & 6.0 & L & Gyrus frontalis inferior & 13 & 18297 & $<0,003132$ \\
\hline 29 & -42.0 & 24.0 & 8.0 & L & Gyrus frontalis inferior & 13 & 13004 & $<0,017544$ \\
\hline 30 & -42.0 & 15.0 & 10.0 & L & Gyrus praecentralis & 44 & 1756 & $<0,000851$ \\
\hline 31 & -48.0 & 18.0 & 5.0 & L & Gyrus frontalis inferior & 45 & 3074 & $<0,000424$ \\
\hline 32 & -42.0 & 21.0 & 14.0 & L & Gyrus frontalis inferior & 45 & 9295 & $<0,000097$ \\
\hline 33 & -29.0 & 16.0 & 10.0 & L & Insel & 13 & 2040 & $<0,003132$ \\
\hline 34 & -34.0 & 29.0 & 8.0 & L & Gyrus frontalis inferior & 45 & 7118 & $<0,010195$ \\
\hline 35 & -38.0 & 18.0 & 9.0 & L & Insel & 13 & 5522 & $<0,000097$ \\
\hline
\end{tabular}

\begin{tabular}{|l|l|l|l|l|l|l|l|l|}
\hline \multicolumn{7}{|l|}{ Tabelle 16: Lage der Aktivitätsschwerpunkte der ROI „Satz Broca“ der rechten Hemisphäre; Gruppe E } \\
\hline Proband & $\mathrm{X}$ & $\mathrm{Y}$ & $\mathrm{Z}$ & Seite & Gyrus & BA & Voxel & p-Wert \\
\hline 24 & 52.0 & 27.0 & 11.0 & $\mathrm{R}$ & Gyrus frontalis inferior & 46 & 436 & $<0,005740$ \\
25 & 46.0 & 6.0 & 27.0 & $\mathrm{R}$ & Gyrus frontalis inferior & 9 & 4482 & $<0,003132$ \\
26 & 53.0 & 18.0 & 14.0 & $\mathrm{R}$ & Gyrus frontalis inferior & 45 & 7243 & $<0,000424$ \\
27 & 42.0 & 28.0 & 13.0 & $\mathrm{R}$ & Gyrus frontalis medialis & 46 & 5270 & $<0,000020$ \\
28 & 43.0 & 29.0 & 14.0 & $\mathrm{R}$ & Gyrus frontalis medialis & 46 & 8689 & $<0,003132$ \\
29 & 31.0 & 21.0 & 17.0 & $\mathrm{R}$ & Insel & 13 & 3805 & $<0,017544$ \\
30 & 44.0 & 16.0 & 4.0 & $\mathrm{R}$ & Insel & 13 & 4088 & $<0,000851$ \\
31 & 45.0 & 33.0 & 24.0 & $\mathrm{R}$ & Gyrus frontalis medialis & 46 & 1045 & $<0,000424$ \\
32 & 34.0 & 18.0 & 5.0 & $\mathrm{R}$ & Insel & 13 & 1703 & $<0,000097$ \\
33 & 44.0 & 14.0 & 13.0 & $\mathrm{R}$ & Gyrus frontalis inferior & 44 & 6708 & $<0,003132$ \\
34 & 40.0 & 24.0 & 3.0 & $\mathrm{R}$ & Gyrus frontalis inferior & 45 & 1822 & $<0,010195$ \\
35 & 36.0 & 19.0 & 11.0 & $\mathrm{R}$ & Insel & 13 & 3219 & $<0,000097$ \\
\hline
\end{tabular}




\begin{tabular}{|c|c|c|c|c|c|}
\hline \multicolumn{6}{|c|}{ Tabelle 17: Vergleich der LIs der ROI „Satz Broca“ } \\
\hline Proband & Gruppe & Voxel links & Voxel rechts & LI ROI „Satz Broca“ & Lateralisierung \\
\hline 1 & $\mathrm{~N}$ & 6316 & 0 & 1 & L \\
\hline 2 & $\mathrm{~N}$ & 12508 & 0 & 1 & L \\
\hline 3 & $\mathrm{~N}$ & 6694 & 3327 & 0,335994412 & L \\
\hline 4 & $\mathrm{~N}$ & 6566 & 0 & 1 & L \\
\hline 5 & $\mathrm{~N}$ & 3769 & 0 & 1 & L \\
\hline 6 & $\mathrm{~N}$ & 8032 & 0 & 1 & L \\
\hline 7 & $\mathrm{~N}$ & 987 & 0 & 1 & $\mathrm{~L}$ \\
\hline 8 & $\mathrm{~N}$ & 5197 & 762 & 0,744252391 & L \\
\hline 9 & $\mathrm{~N}$ & 2514 & 1542 & 0,23964497 & L \\
\hline 10 & $\mathrm{~N}$ & 6701 & 1843 & 0,568586142 & L \\
\hline 11 & N & 7725 & 1405 & 0,692223439 & L \\
\hline 12 & $\mathrm{~N}$ & 2439 & 0 & 1 & L \\
\hline 13 & $N$ & 1956 & 0 & 1 & L \\
\hline 14 & $\mathrm{~N}$ & 3034 & 0 & 1 & L \\
\hline 15 & $\mathrm{~N}$ & 5633 & 0 & 1 & L \\
\hline 16 & N & 7864 & 0 & 1 & L \\
\hline 17 & $N$ & 430 & 3772 & $-0,795335554$ & $R$ \\
\hline 18 & $\mathrm{~N}$ & 0 & 0 & & \\
\hline 19 & $N$ & 3460 & 0 & 1 & L \\
\hline 20 & $\mathrm{~N}$ & 6418 & 811 & 0,775625951 & L \\
\hline 21 & $\mathrm{~N}$ & 10941 & 2381 & 0,642546164 & L \\
\hline 22 & $N$ & 5624 & 0 & 1 & L \\
\hline 23 & $\mathrm{~N}$ & 3642 & 0 & 1 & L \\
\hline 24 & $E$ & 2374 & 436 & 0,689679715 & $L$ \\
\hline 25 & E & 5043 & 4482 & 0,058897638 & s \\
\hline 26 & E & 6441 & 7243 & $-0,058608594$ & s \\
\hline 27 & $E$ & 12898 & 5270 & 0,419859093 & L \\
\hline 28 & E & 18297 & 8689 & 0,356036463 & L \\
\hline 29 & E & 13004 & 3805 & 0,547266345 & L \\
\hline 30 & E & 1756 & 4088 & $-0,399041752$ & $R$ \\
\hline 31 & $E$ & 3074 & 1045 & 0,49259529 & L \\
\hline 32 & E & 9295 & 1703 & 0,690307329 & L \\
\hline 33 & E & 2040 & 6708 & $-0,533607682$ & $R$ \\
\hline 34 & E & 7118 & 1822 & 0,592393736 & L \\
\hline 35 & E & 5522 & 3219 & 0,263470999 & L \\
\hline
\end{tabular}




\begin{tabular}{|c|c|c|c|c|c|c|c|c|}
\hline Proband & $x$ & $\mathrm{Y}$ & $z$ & Seite & Gyrus & $B A$ & Voxel & $p$-Wert \\
\hline 1 & -56.0 & -38.0 & 12.0 & L & Gyrus temporalis superior & 22 & 1763 & $<0,000168$ \\
\hline 2 & -61.0 & -21.0 & -1.0 & L & Gyrus temporalis superior & 21 & 3711 & $<0,000066$ \\
\hline 3 & -53.0 & -33.0 & 5.0 & L & Gyrus temporalis medialis & 22 & 8035 & $<0,000066$ \\
\hline 4 & -53.0 & -37.0 & 11.0 & L & Gyrus temporalis superior & 22 & 1593 & $<0,000001$ \\
\hline 5 & -57.0 & -39.0 & 15.0 & L & Gyrus temporalis superior & 22 & 3916 & $<0,000001$ \\
\hline 6 & -56.0 & -29.0 & 1.0 & L & Gyrus temporalis medialis & 21 & 2211 & $<1,2738 \cdot 10^{-08}$ \\
\hline 7 & -48.0 & -29.0 & 19.0 & L & Insel & 13 & 463 & $<0,022423$ \\
\hline 8 & & & & & & & 0 & $<0,001274$ \\
\hline 9 & -53.0 & -34.0 & 13.0 & L & Gyrus temporalis superior & 42 & 331 & $<0,047192$ \\
\hline 10 & -57.0 & -43.0 & 4.0 & L & Gyrus temporalis medialis & 22 & 6263 & $<0,000097$ \\
\hline 11 & -54.0 & -33.0 & 3.0 & L & Gyrus temporalis medialis & 22 & 3391 & $<0,000097$ \\
\hline 12 & -57.0 & -35.0 & 8.0 & L & Gyrus temporalis superior & 22 & 3034 & $<6,6637 \cdot 10^{-11}$ \\
\hline 13 & -57.0 & -41.0 & 3.0 & L & Gyrus frontalis medialis & 22 & 2383 & $<6,6637 \cdot 10^{-11}$ \\
\hline 14 & -55.0 & -25.0 & -3.0 & L & Gyrus temporalis superior & 21 & 638 & $<0,000001$ \\
\hline 15 & -51.0 & -30.0 & 0.0 & L & Gyrus temporalis medialis & 21 & 1225 & $<6,6637 \cdot 10^{-11}$ \\
\hline 16 & -47.0 & -26.0 & 2.0 & L & Gyrus temporalis superior & 22 & 324 & $<0,000044$ \\
\hline 17 & -55.0 & -37.0 & 7.0 & L & Gyrus temporalis medialis & 22 & 3117 & $<0,000424$ \\
\hline 18 & -62.0 & -24.0 & 11.0 & L & Gyrus temporalis superior & 42 & 283 & $<0,047192$ \\
\hline 19 & -48.0 & -44.0 & 5.0 & L & Gyrus temporalis medialis & 22 & 883 & $<6,6637 \cdot 10^{-11}$ \\
\hline 20 & -49.0 & -39.0 & 0.0 & L & Gyrus temporalis medialis & 22 & 3990 & 0,000001 \\
\hline 21 & -63.0 & -24.0 & 1.0 & L & Gyrus temporalis superior & 22 & 2111 & $<0,003132$ \\
\hline 22 & -59.0 & -27.0 & 9.0 & L & Gyrus temporalis superior & 42 & 949 & $<7,5117 \cdot 10^{-13}$ \\
\hline 23 & -62.0 & -32.0 & 6.0 & L & Gyrus temporalis superior & 22 & 1264 & $<0,003132$ \\
\hline
\end{tabular}




\begin{tabular}{|c|c|c|c|c|c|c|c|c|}
\hline Proband & $x$ & $\mathrm{Y}$ & Z & Seite & Gyrus & BA & Voxel & p-Wert \\
\hline 1 & 46.0 & -31.0 & 9.0 & $\mathrm{R}$ & Gyrus temporalis superior & 41 & 2013 & $<0,000168$ \\
\hline 2 & 59.0 & -31.0 & 3.0 & $\mathrm{R}$ & Gyrus temporalis medialis & 22 & 3008 & $<0,000066$ \\
\hline 3 & 50.0 & -33.0 & 4.0 & $\mathrm{R}$ & Gyrus temporalis superior & 22 & 8031 & $<0,000066$ \\
\hline 4 & & & & & & & 0 & $<0,000001$ \\
\hline 5 & 63.0 & -32.0 & 21.0 & $\mathrm{R}$ & Gyrus temporalis superior & 42 & 288 & $<0,000001$ \\
\hline 6 & 49.0 & -27.0 & -3.0 & $R$ & Gyrus temporalis superior & 21 & 545 & $<1,2738 \cdot 10^{-08}$ \\
\hline 7 & & & & & & & 0 & $<0,022423$ \\
\hline 8 & 47.0 & -27.0 & -1.0 & $\mathrm{R}$ & Gyrus temporalis superior & 22 & 1787 & $<0,001274$ \\
\hline 9 & 47.0 & -33.0 & 5.0 & $\mathrm{R}$ & Gyrus temporalis superior & 22 & 1036 & $<0,047192$ \\
\hline 10 & 49.0 & -31.0 & 7.0 & $\mathrm{R}$ & Gyrus temporalis superior & 22 & 3265 & $<0,000097$ \\
\hline 11 & 53.0 & -30.0 & 5.0 & $\mathrm{R}$ & Gyrus temporalis superior & 22 & 2680 & $<0,000097$ \\
\hline 12 & & & & & & & 0 & $<6,6637 \cdot 10^{-11}$ \\
\hline 13 & & & & & & & 0 & $<6,6637 \cdot 10^{-11}$ \\
\hline 14 & & & & & & & 0 & $<0,000001$ \\
\hline 15 & & & & & & & 0 & $<6,6637 \cdot 10^{-11}$ \\
\hline 16 & 45.0 & -26.0 & 0.0 & $\mathrm{R}$ & Gyrus temporalis superior & 22 & 488 & $<0,000044$ \\
\hline 17 & 46.0 & -27.0 & -1.0 & $\mathrm{R}$ & Gyrus temporalis superior & 22 & 998 & $<0,000424$ \\
\hline 18 & & & & & & & 0 & $<0,047192$ \\
\hline 19 & & & & & & & 0 & $<6,6637 \cdot 10^{-11}$ \\
\hline 20 & 48.0 & -27.0 & 2.0 & $\mathrm{R}$ & Gyrus temporalis superior & 22 & 557 & 0,000001 \\
\hline 21 & & & & & & & 0 & $<0,003132$ \\
\hline 22 & & & & & & & 0 & $<7,5117 \cdot 10^{-13}$ \\
\hline 23 & 51.0 & -31.0 & 3.0 & $\mathrm{R}$ & Gyrus temporalis superior & 22 & 299 & $<0,003132$ \\
\hline \multicolumn{9}{|c|}{$\begin{array}{l}\text { Wichtige Informationen über die ausgewertete ROI im Gebiet der Wernicke-Region der rechten Hemisphäre der } \\
\text { funktionellen MRT-Daten: Talairachkoordinaten des Aktivitätsschwerpunktes, Seitenlokalisation der Aktivierung, } \\
\text { anatomische Lage und zugehöriges BA des Aktivitätsschwerpunktes, Anzahl der signifikant aktivierten Voxel, statistische } \\
\text { Schwelle }\end{array}$} \\
\hline
\end{tabular}




\begin{tabular}{|c|c|c|c|c|c|c|c|c|}
\hline Proband & $x$ & $\mathrm{Y}$ & Z & Seite & Gyrus & BA & Voxel & p-Wert \\
\hline 24 & -48.0 & -34.0 & 9.0 & L & Gyrus temporalis superior & 41 & 1175 & $<0,005740$ \\
\hline 25 & -56.0 & -48.0 & 10.0 & L & Gyrus temporalis superior & 22 & 5408 & $<0,003132$ \\
\hline 26 & -50.0 & -42.0 & 2.0 & L & Gyrus temporalis medialis & 22 & 3547 & $<0,000424$ \\
\hline 27 & -47.0 & -38.0 & 10.0 & L & Gyrus temporalis superior & 41 & 517 & $<0,000020$ \\
\hline 28 & -56.0 & -35.0 & 1.0 & L & Gyrus temporalis medialis & 22 & 9026 & $<0,003132$ \\
\hline 29 & -47.0 & -42.0 & -3.0 & L & Sub-Gyral & 37 & 4956 & $<0,017544$ \\
\hline 30 & & & & & & & 0 & $<0,000851$ \\
\hline 31 & -53.0 & -33.0 & 10.0 & L & Gyrus temporalis superior & 41 & 4545 & $<0,000424$ \\
\hline 32 & -54.0 & -31.0 & 10.0 & L & Gyrus temporalis superior & 41 & 3504 & $<0,000097$ \\
\hline 33 & -46.0 & -44.0 & 6.0 & L & Gyrus temporalis medialis & 22 & 2881 & $<0,003132$ \\
\hline 34 & -48.0 & -38.0 & -5.0 & L & ** & & 2134 & $<0,010195$ \\
\hline 35 & -52.0 & -40.0 & 17.0 & L & Gyrus temporalis superior & 13 & 2227 & $<0,000097$ \\
\hline \multicolumn{9}{|c|}{$\begin{array}{l}\text { Wichtige Informationen über die ausgewertete ROI im Gebiet der Wernicke-Region der linken Hemisphäre der funktioneller } \\
\text { MRT-Daten: Talairachkoordinaten des Aktivitätsschwerpunktes, Seitenlokalisation der Aktivierung, anatomische Lage und } \\
\text { zugehöriges BA des Aktivitätsschwerpunktes, Anzahl der signifikant aktivierten Voxel, statistische Schwelle } \\
\text { ** Aktivitätsschwerpunkt konnte keine graue Substanz zugeordnet werden }\end{array}$} \\
\hline
\end{tabular}

\begin{tabular}{|c|c|c|c|c|c|c|c|c|}
\hline Proband & $x$ & $\mathrm{Y}$ & Z & Seite & Gyrus & BA & Voxel & $p$-Wert \\
\hline 24 & 49.0 & -22.0 & 2.0 & $\mathrm{R}$ & Gyrus temporalis superior & 22 & 1999 & $<0,005740$ \\
\hline 25 & 46.0 & -36.0 & -3.0 & $\mathrm{R}$ & Gyrus temporalis medialis & * & 4713 & $<0,003132$ \\
\hline 26 & 55.0 & -46.0 & 10.0 & $\mathrm{R}$ & Gyrus temporalis superior & 22 & 4326 & $<0,000424$ \\
\hline 27 & 43.0 & -37.0 & 4.0 & $\mathrm{R}$ & Gyrus temporalis superior & 41 & 1260 & $<0,000020$ \\
\hline 28 & & & & & & & 0 & $<0,003132$ \\
\hline 29 & & & & & & & 0 & $<0,017544$ \\
\hline 30 & 44.0 & -37.0 & 10.0 & $\mathrm{R}$ & Gyrus temporalis superior & 41 & 351 & $<0,000851$ \\
\hline 31 & 53.0 & -29.0 & 10.0 & $\mathrm{R}$ & Gyrus temporalis superior & 41 & 1044 & $<0,000424$ \\
\hline 32 & 46.0 & -26.0 & 2.0 & $\mathrm{R}$ & Gyrus temporalis superior & 22 & 421 & $<0,000097$ \\
\hline 33 & 53.0 & -31.0 & -2.0 & $\mathrm{R}$ & Gyrus temporalis medialis & 21 & 2268 & $<0,003132$ \\
\hline 34 & & & & & & & 0 & $<0,010195$ \\
\hline 35 & 54.0 & -36.0 & 5.0 & $\mathrm{R}$ & Gyrus temporalis medialis & 22 & 3914 & $<0,000097$ \\
\hline \multicolumn{9}{|c|}{$\begin{array}{l}\text { Wichtige Informationen über die ausgewertete ROI im Gebiet der Wernicke-Region der rechten Hemisphäre der } \\
\text { funktionellen MRT-Daten: Talairachkoordinaten des Aktivitätsschwerpunktes, Seitenlokalisation der Aktivierung, } \\
\text { anatomische Lage und zugehöriges BA des Aktivitätsschwerpunktes, Anzahl der signifikant aktivierten Voxel, statistische } \\
\text { Schwelle }\end{array}$} \\
\hline
\end{tabular}




\begin{tabular}{|c|c|c|c|c|c|}
\hline Proband & Gruppe & Voxel links & Voxel rechts & LI ROI „Satz Wernicke“ & Lateralisierung \\
\hline 1 & $N$ & 1763 & 2013 & $\mid-0,066207627$ & $\mathrm{~s}$ \\
\hline 2 & $\mathrm{~N}$ & 3711 & 3008 & 0,104628665 & L \\
\hline 3 & $\mathrm{~N}$ & 8035 & 8031 & 0,000248973 & $S$ \\
\hline 4 & $\mathrm{~N}$ & 1593 & 0 & 1 & $\mathrm{~L}$ \\
\hline 5 & $\mathrm{~N}$ & 3916 & 288 & 0,862987631 & $\mathrm{L}$ \\
\hline 6 & $\mathrm{~N}$ & 2211 & 545 & 0,604499274 & L \\
\hline 7 & $\mathrm{~N}$ & 463 & 0 & 1 & L \\
\hline 8 & $\mathrm{~N}$ & 0 & 1787 & -1 & $\mathrm{R}$ \\
\hline 9 & $\mathrm{~N}$ & 331 & 1036 & $-0,515727871$ & $\mathrm{R}$ \\
\hline 10 & $\mathrm{~N}$ & 6263 & 3265 & 0,314651553 & L \\
\hline 11 & $\mathrm{~N}$ & 3391 & 2680 & 0,117114149 & L \\
\hline 12 & $\mathrm{~N}$ & 3034 & 0 & 1 & L \\
\hline 13 & $\mathrm{~N}$ & 2383 & 0 & 1 & L \\
\hline 14 & $\mathrm{~N}$ & 638 & 0 & 1 & L \\
\hline 15 & $\mathrm{~N}$ & 1225 & 0 & 1 & $\mathrm{~L}$ \\
\hline 16 & $\mathrm{~N}$ & 324 & 488 & $\mid-0,201970443$ & $\mathrm{R}$ \\
\hline 17 & N & 3117 & 998 & 0,514945322 & L \\
\hline 18 & $\mathrm{~N}$ & 283 & 0 & 1 & L \\
\hline 19 & $\mathrm{~N}$ & 883 & 0 & 1 & $\mathrm{~L}$ \\
\hline 20 & $\mathrm{~N}$ & 3990 & 557 & 0,755003299 & $\mathrm{~L}$ \\
\hline 21 & $\mathrm{~N}$ & 2111 & 0 & 1 & L \\
\hline 22 & N & 949 & 0 & 1 & L \\
\hline 23 & $N$ & 1264 & 299 & 0,617402431 & L \\
\hline 24 & E & 1175 & 1999 & $-0,259609326$ & $\mathrm{R}$ \\
\hline 25 & E & 5408 & 4713 & 0,068669104 & $S$ \\
\hline 26 & E & 3547 & 4326 & -0,098945764 & S \\
\hline 27 & E & 517 & 1260 & $-0,418120428$ & $R$ \\
\hline 28 & E & 9026 & 0 & 1 & $\mathrm{~L}$ \\
\hline 29 & E & 4956 & 0 & 1 & L \\
\hline 30 & E & 0 & 351 & -1 & $\mathrm{R}$ \\
\hline 31 & E & 4545 & 1044 & 0,626409018 & L \\
\hline 32 & E & 3504 & 421 & 0,785477707 & L \\
\hline 33 & E & 2881 & 2268 & 0,119052243 & $\mathrm{~L}$ \\
\hline 34 & E & 2134 & 0 & 1 & L \\
\hline 35 & E & 2227 & 3914 & $\mid-0,274710959$ & $R$ \\
\hline
\end{tabular}




\begin{tabular}{|l|l|l|l|l|}
\hline Tabelle 23: Deskriptive Statistik der LIs der ROI „Wort Broca“ \\
\hline & Gültige Anzahl & Median & Minimum & Maximum \\
\hline Lateralisierung links Gruppe N & 20 & 0,915 & 0,146 & 1,000 \\
Lateralisierung links Gruppe E & 8 & 0,638 & 0,164 & 1,000 \\
\hline Lateralisierung rechts Gruppe N & 2 & $-0,242$ & $-0,346$ & $-0,137$ \\
Lateralisierung rechts Gruppe E & 3 & $-0,169$ & $-0,760$ & $-0,138$ \\
\hline Lateralisierung symmetrisch Gruppe E & 1 & 0,006 & & \\
\hline
\end{tabular}

\begin{tabular}{|l|l|l|l|l|}
\hline Tabelle 24: Deskriptive Statistik: LIs der ROI „Wort Wernicke“ \\
\hline & Gültige Anzahl & Median & Minimum & Maximum \\
\hline Lateralisierung links Gruppe N & 16 & 1,000 & 0,194 & 1,000 \\
Lateralisierung links Gruppe E & 9 & 0,648 & 0,117 & 1,000 \\
\hline Lateralisierung rechts Gruppe N & 6 & $-0,336$ & $-1,000$ & $-0,103$ \\
Lateralisierung rechts Gruppe E & 3 & $-0,266$ & $-0,423$ & $-0,250$ \\
\hline Lateralisierung symmetrisch Gruppe N & 1 & $-0,007$ & & \\
\hline
\end{tabular}

\begin{tabular}{|l|l|l|l|l|}
\hline Tabelle 25: Deskriptive Statistik LIs der ROI „Satz Broca“ \\
\hline & Gültige Anzahl & Median & Minimum & Maximum \\
\hline Lateralisierung links Gruppe N & 21 & 1,000 & 0,240 & 1,000 \\
Lateralisierung links Gruppe E & 8 & 0,520 & 0,263 & 0,690 \\
\hline Lateralisierung rechts Gruppe N & 1 & $-0,795336$ & & \\
Lateralisierung rechts Gruppe E & 2 & $-0,466$ & $-0,534$ & $-0,399$ \\
\hline Lateralisierung symmetrisch Gruppe E & 2 & 0,00014 & $-0,0586$ & 0,0589 \\
\hline
\end{tabular}

\begin{tabular}{|l|l|l|l|l|}
\hline \multicolumn{4}{|l|}{ Tabelle 26: Deskriptive Statistik der LIs der ROI „Satz Wernicke“ } \\
\hline & Gültige Anzahl & Median & Minimum & Maximum \\
\hline Lateralisierung links Gruppe N & 18 & 1,000 & 0,105 & 1,000 \\
Lateralisierung links Gruppe E & 6 & 0,893 & 0,119 & 1,000 \\
\hline Lateralisierung rechts Gruppe N & 3 & $-0,516$ & $-1,000$ & $-0,202$ \\
Lateralisierung rechts Gruppe E & 4 & $-0,346$ & $-1,000$ & $-0,260$ \\
\hline Lateralisierung symmetrisch Gruppe N & 2 & $-0,0330$ & $-0,0662$ & 0,00025 \\
Lateralisierung symmetrisch Gruppe E & 2 & $-0,0151$ & $-0,0989$ & 0,0687 \\
\hline
\end{tabular}




\begin{tabular}{|l|l|l|l|}
\hline Tabelle 27: Mann-Whitney U-Test der LIs für die Gruppe N gegen E \\
\hline & $p$-Wert & Gültige Anzahl Gruppe N & Gültige Anzahl Gruppe E \\
\hline LI ROI „Wort Broca“ & 0,040951 & 22 & 12 \\
\hline LI ROI „Satz Broca“ & 0,000201 & 22 & 12 \\
\hline LI ROI „Wort Wernicke“ & 0,604943 & 23 & 12 \\
\hline LI ROI „Satz Wernicke“ & 0,164113 & 23 & 12 \\
\hline gruppiert nach Gruppe, Test signifikant ab p <0,05 \\
\hline
\end{tabular}

Tabelle 28: Mann-Whitney U-Test der Lls für die Gruppe N gegen E links

\begin{tabular}{|l|l|l|l|}
\hline & $p-W e r t$ & Gültige Anzahl Gruppe N & Gültige Anzahl Gruppe E links \\
\hline LI ROI „Wort Broca“ & 0,017702 & 22 & 9 \\
\hline LI ROI „Satz Broca“ & 0,001017 & 22 & 9 \\
\hline LI ROI „Wort Wernicke“ & 0,801445 & 23 & 9 \\
\hline LI ROI „Satz Wernicke“ & 0,345660 & 23 & 9 \\
\hline \multicolumn{2}{|l}{ gruppiert nach Gruppe, Test signifikant ab p <0,05 } \\
\hline
\end{tabular}




\begin{tabular}{|c|c|c|c|c|c|}
\hline Proband & Gruppe & FA mean links & FA mean rechts & AI ROI „Wort Broca“ & Verteilung der Als \\
\hline 1 & $N$ & 0,185262 & 0,336471 & $-0,289820655$ & $R$ \\
\hline 2 & $N$ & 0,293555 & 0,265178 & 0,050788122 & $S$ \\
\hline 3 & $N$ & 0,246723 & 0,146739 & 0,254113485 & $L$ \\
\hline 4 & $N$ & 0,184243 & 0 & 1 & $L$ \\
\hline 5 & $N$ & 0,181599 & 0 & 1 & $L$ \\
\hline 6 & $N$ & 0,264545 & 0 & 1 & $L$ \\
\hline 7 & $N$ & 0,286633 & 0,099863 & 0,483239154 & $L$ \\
\hline 8 & $N$ & 0,381447 & 0,185311 & 0,346066575 & $L$ \\
\hline 9 & $N$ & 0,35676 & 0,404523 & $-0,062740137$ & $S$ \\
\hline 10 & $N$ & 0,134353 & 0,207976 & $-0,21506504$ & $R$ \\
\hline 11 & $N$ & 0,230042 & 0,137148 & 0,252986192 & L \\
\hline 12 & $N$ & 0,306926 & 0 & 1 & $L$ \\
\hline 13 & $N$ & 0,136472 & 0 & 1 & L \\
\hline 14 & $N$ & 0,335679 & 0,279149 & 0,091944414 & $S$ \\
\hline 15 & $N$ & 0,352494 & 0 & 1 & L \\
\hline 16 & $N$ & 0,281949 & 0 & 1 & $L$ \\
\hline 17 & $N$ & 0,350373 & 0,293575 & 0,088202774 & $S$ \\
\hline 18 & $N$ & 0,267772 & 0 & 1 & L \\
\hline 19 & $N$ & 0,281893 & 0 & 1 & $L$ \\
\hline 20 & $N$ & 0,295202 & 0,168491 & 0,273264854 & $L$ \\
\hline 21 & $N$ & 0,342132 & 0,269648 & 0,1184805 & $L$ \\
\hline 22 & $N$ & 0,27438 & 0 & 1 & $L$ \\
\hline 23 & $N$ & 0,309817 & 0 & 1 & L \\
\hline 24 & $E$ & 0,332821 & 0,322719 & 0,015410196 & $S$ \\
\hline 25 & $E$ & 0,332568 & 0,25102 & 0,139735567 & $L$ \\
\hline 26 & $E$ & 0,28231 & 0,312952 & $-0,051476493$ & $S$ \\
\hline 27 & $E$ & 0,459727 & 0,379766 & 0,095249156 & $S$ \\
\hline 28 & $E$ & 0,362166 & 0,248606 & 0,185928628 & L \\
\hline 29 & $E$ & 0,332632 & 0,20138 & 0,245784739 & L \\
\hline 30 & $E$ & 0,342198 & 0,425648 & $-0,108680647$ & $R$ \\
\hline 31 & $E$ & 0,377522 & 0 & 1 & L \\
\hline 32 & $E$ & 0,280997 & 0,181495 & 0,215143181 & L \\
\hline 33 & $E$ & 0,200506 & 0,279233 & $-0,164103815$ & $\mathrm{R}$ \\
\hline 34 & $E$ & 0,414928 & 0,23961 & 0,267849995 & L \\
\hline 35 & $E$ & 0,063239 & 0,198229 & $-0,516277326$ & $R$ \\
\hline
\end{tabular}




\begin{tabular}{|c|c|c|c|c|c|}
\hline Proband & Gruppe & FA mean links & FA mean rechts & Al ROI "Satz Broca“ & Verteilung der Als \\
\hline 1 & $\mathrm{~N}$ & 0,43068 & 0 & 1 & L \\
\hline 2 & $\mathrm{~N}$ & 0,293555 & 0 & 1 & L \\
\hline 3 & $\mathrm{~N}$ & 0,396367 & 0,343523 & 0,071421428 & $S$ \\
\hline 4 & N & 0,220099 & 0 & 1 & L \\
\hline 5 & $\mathrm{~N}$ & 0,166697 & 0 & 1 & L \\
\hline 6 & $\mathrm{~N}$ & 0,25493 & 0 & 1 & L \\
\hline 7 & $\mathrm{~N}$ & 0,106482 & 0 & 1 & $\mathrm{~L}$ \\
\hline 8 & $\mathrm{~N}$ & 0,397952 & 0,066213 & | 0,714700591 & $\mathrm{~L}$ \\
\hline 9 & $\mathrm{~N}$ & 0,333209 & 0,117021 & 0,480172356 & L \\
\hline 10 & $N$ & 0,390947 & 0,2081 & 0,305229807 & L \\
\hline 11 & $N$ & 0,261812 & 0,25379 & 0,015558512 & $S$ \\
\hline 12 & N & 0,117173 & 0 & 1 & L \\
\hline 13 & $\mathrm{~N}$ & 0,133845 & 0 & 1 & L \\
\hline 14 & $N$ & 0,284652 & 0 & 1 & L \\
\hline 15 & $\mathrm{~N}$ & 0,272192 & 0 & 1 & L \\
\hline 16 & $N$ & 0,286353 & 0 & 1 & L \\
\hline 17 & N & 0 & 0,302872 & -1 & $R$ \\
\hline 18 & N & 0,213449 & 0,324002 & $\mid-0,205698752$ & $R$ \\
\hline 19 & $N$ & 0,274612 & 0 & 1 & L \\
\hline 20 & $N$ & 0,286001 & 0,158783 & 0,286021979 & L \\
\hline 21 & $N$ & 0,246692 & 0,135535 & 0,290814097 & L \\
\hline 22 & N & 0,27438 & 0 & 1 & L \\
\hline 23 & $N$ & 0,290168 & 0 & 1 & L \\
\hline 24 & $E$ & 0,1279 & 0,160324 & $\mid-0,112495837$ & $R$ \\
\hline 25 & $E$ & 0,434912 & 0,403439 & 0,037541555 & $S$ \\
\hline 26 & $E$ & 0,27729 & 0,276727 & 0,001016214 & $S$ \\
\hline 27 & $E$ & 0,459727 & 0,367425 & 0,111590131 & L \\
\hline 28 & E & 0,367257 & 0,394627 & $-0,035924104$ & S \\
\hline 29 & $E$ & 0,283948 & 0,290255 & $\mid-0,01098392$ & $S$ \\
\hline 30 & $E$ & 0,254293 & 0,214905 & 0,083947502 & $S$ \\
\hline 31 & $E$ & 0,115392 & 0,253143 & $\mid-0,373779967$ & $R$ \\
\hline 32 & $E$ & 0,367718 & 0,148134 & 0,42567248 & L \\
\hline 33 & E & 0,316081 & 0,345751 & $-0,044830108$ & S \\
\hline 34 & $E$ & 0,329954 & 0,194907 & 0,257300504 & L \\
\hline 35 & E & 0,198879 & 0,353177 & $\mid-0,279497008$ & $\mathrm{R}$ \\
\hline
\end{tabular}




\begin{tabular}{|c|c|c|c|c|c|}
\hline Proband & Gruppe & FA mean links & FA mean rechts & Al ROI „Wort Wernicke“ & Verteilung der Als \\
\hline 1 & $\mathrm{~N}$ & 0,174425 & 0,37621 & $-0,366458725$ & $\mathrm{R}$ \\
\hline 2 & $\mathrm{~N}$ & 0,19162 & 0,297667 & $-0,216737825$ & $\mathrm{R}$ \\
\hline 3 & $\mathrm{~N}$ & 0,33027 & 0,315015 & 0,023640717 & $\mathrm{~s}$ \\
\hline 4 & $\mathrm{~N}$ & 0,178777 & 0 & 1 & L \\
\hline 5 & N & 0,3107 & 0,178936 & 0,26910603 & L \\
\hline 6 & $N$ & 0,230403 & 0 & 1 & L \\
\hline 7 & N & 0,278741 & 0,188738 & 0,192528434 & L \\
\hline 8 & N & 0 & 0,196524 & -1 & $R$ \\
\hline 9 & $\mathrm{~N}$ & 0,17707 & 0,364517 & $-0,346106904$ & $\mathrm{R}$ \\
\hline 10 & $N$ & 0,264027 & 0,3794 & $\mid-0,179310163$ & $R$ \\
\hline 11 & $N$ & 0,215327 & 0,203035 & 0,029381254 & $S$ \\
\hline 12 & N & 0,252994 & 0 & 1 & L \\
\hline 13 & N & 0,294631 & 0 & 1 & L \\
\hline 14 & $N$ & 0,238227 & 0 & 1 & L \\
\hline 15 & $N$ & 0,202661 & 0 & 1 & L \\
\hline 16 & $N$ & 0,333968 & 0 & 1 & L \\
\hline 17 & N & 0 & 0,114141 & -1 & $R$ \\
\hline 18 & N & 0,178676 & 0,174821 & 0,010905326 & $S$ \\
\hline 19 & $N$ & 0,195187 & 0 & 1 & L \\
\hline 20 & $N$ & 0,220253 & 0,229886 & $\mid-0,021400056$ & $S$ \\
\hline 21 & $N$ & 0,118044 & 0 & 1 & L \\
\hline 22 & N & 0,113776 & 0 & 1 & L \\
\hline 23 & $N$ & 0,194014 & 0 & 1 & L \\
\hline 24 & $E$ & 0,245017 & 0,29033 & $\mid-0,084642297$ & $S$ \\
\hline 25 & $E$ & 0,111822 & 0,319464 & $\mid-0,481448505$ & $R$ \\
\hline 26 & $E$ & 0,231625 & 0,230922 & 0,001519846 & $S$ \\
\hline 27 & $E$ & 0,339256 & 0 & 1 & L \\
\hline 28 & E & 0,254378 & 0 & 1 & L \\
\hline 29 & $E$ & 0,134119 & 0 & 1 & L \\
\hline 30 & $E$ & 0,138844 & 0,178587 & $\mid-0,125202012$ & $R$ \\
\hline 31 & $E$ & 0,225772 & 0,22217 & 0,00804122 & $S$ \\
\hline 32 & $E$ & 0,18813 & 0,147293 & 0,121747763 & L \\
\hline 33 & $E$ & 0,313941 & 0,332418 & $\mid-0,028586281$ & $S$ \\
\hline 34 & $E$ & 0,249459 & 0,224156 & 0,05342525 & $S$ \\
\hline 35 & E & 0,087885 & 0,150066 & $-0,261318507$ & $\mathrm{R}$ \\
\hline
\end{tabular}




\begin{tabular}{|c|c|c|c|c|c|}
\hline Proband & Gruppe & FA mean links & FA mean rechts & Al ROI „Satz Wernicke“ & Verteilung der Als \\
\hline 1 & $N$ & 0,227627 & 0,227987 & $-0,000790143$ & $S$ \\
\hline 2 & $N$ & 0,407373 & 0,297667 & 0,155602519 & $\mathrm{~L}$ \\
\hline 3 & $\mathrm{~N}$ & 0,33027 & 0,286873 & 0,070319197 & $S$ \\
\hline 4 & $N$ & 0,215854 & 0 & 1 & L \\
\hline 5 & $N$ & 0,275833 & 0,100629 & 0,465396242 & $\mathrm{~L}$ \\
\hline 6 & $\mathrm{~N}$ & 0,336282 & 0,178488 & 0,306533015 & $L$ \\
\hline 7 & $\mathrm{~N}$ & 0,162631 & 0 & 1 & $\mathrm{~L}$ \\
\hline 8 & $N$ & 0 & 0,241979 & -1 & $R$ \\
\hline 9 & $N$ & 0,280536 & 0,278835 & 0,003040916 & $S$ \\
\hline 10 & $N$ & 0,273037 & 0,394144 & $-0,181520457$ & $R$ \\
\hline 11 & $N$ & 0,176765 & 0,246591 & $-0,164934476$ & $R$ \\
\hline 12 & $N$ & 0,226214 & 0 & 1 & L \\
\hline 13 & $N$ & 0,235522 & 0 & 1 & L \\
\hline 14 & $N$ & 0,26701 & 0 & 1 & $\mathrm{~L}$ \\
\hline 15 & $N$ & 0,192207 & 0 & 1 & $L$ \\
\hline 16 & $N$ & 0,222474 & 0,239206 & $-0,036241553$ & $S$ \\
\hline 17 & $N$ & 0,26871 & 0,234058 & 0,068922445 & $S$ \\
\hline 18 & $N$ & 0,172233 & 0 & 1 & L \\
\hline 19 & $N$ & 0,195187 & 0 & 1 & L \\
\hline 20 & $N$ & 0,176435 & 0,148948 & 0,084475833 & $S$ \\
\hline 21 & $N$ & 0,217413 & 0 & 1 & L \\
\hline 22 & $N$ & 0,114203 & 0 & 1 & L \\
\hline 23 & $N$ & 0,273207 & 0,106046 & 0,440763817 & L \\
\hline 24 & $E$ & 0,22424 & 0,199601 & 0,058132649 & $S$ \\
\hline 25 & $E$ & 0,327055 & 0,363375 & $-0,052604898$ & $S$ \\
\hline 26 & $E$ & 0,17743 & 0,202367 & $-0,06565876$ & $S$ \\
\hline 27 & E & 0,236761 & 0,239999 & $-0,006791677$ & $S$ \\
\hline 28 & $E$ & 0,250612 & 0 & 1 & L \\
\hline 29 & E & 0,254149 & 0 & 1 & L \\
\hline 30 & E & 0 & 0,160996 & -1 & $R$ \\
\hline 31 & E & 0,273035 & 0,105892 & 0,441095514 & L \\
\hline 32 & $E$ & 0,193145 & 0,138247 & 0,165658797 & L \\
\hline 33 & E & 0,315217 & 0,29986 & 0,024967606 & $S$ \\
\hline 34 & $E$ & 0,249459 & 0 & 1 & L \\
\hline 35 & E & 0,184901 & 0,307382 & $-0,24880201$ & $R$ \\
\hline
\end{tabular}




\begin{tabular}{|l|l|l|l|l|}
\hline \multicolumn{2}{|l|}{ Tabelle 33: Deskriptive Statistik der Als der strukturellen ROI „Wort Broca“ } \\
\hline & Gültige Anzahl & Median & Minimum & Maximum \\
\hline Linkshemisphärisch höhere FA Gruppe N & 17 & 1,000 & 0,118 & 1,000 \\
Linkshemisphärisch höhere FA Gruppe E & 6 & 0,230 & 0,140 & 1,000 \\
\hline Rechtshemisphärisch höhere FA Gruppe N & 2 & $-0,252$ & $-0,290$ & $-0,215$ \\
Rechtshemisphärisch höhere FA Gruppe E & 3 & $-0,164$ & $-0,516$ & $-0,109$ \\
\hline Symmetrisch verteilte FA Gruppe N & 4 & 0,0695 & $-0,0627$ & 0,0919 \\
Symmetrisch verteilte FAGruppe E & 3 & 0,0154 & $-0,0515$ & 0,0952 \\
\hline
\end{tabular}

\begin{tabular}{|l|l|l|l|l|}
\hline \multicolumn{2}{|l|}{ Tabelle 34: Deskriptive Statistik der Als der strukturellen ROI „Satz Broca“ } \\
\hline & Gültige Anzahl & Median. & Minimum & Maximum \\
\hline Linkshemisphärisch höhere FA Gruppe N & 19 & 1,000 & 0,286 & 1,000 \\
Linkshemisphärisch höhere FA Gruppe E & 3 & 0,257 & 0,112 & 0,426 \\
\hline Rechtshemisphärisch höhere FA Gruppe N & 2 & $-0,603$ & $-1,000$ & $-0,206$ \\
Rechtshemisphärisch höhere FA Gruppe E & 3 & $-0,279$ & $-0,374$ & $-0,112$ \\
\hline Symmetrisch verteilte FA Gruppe N & 2 & 0,0435 & 0,0156 & 0,0714 \\
Symmetrisch verteilte FA Gruppe E & 6 & $-0,00498$ & $-0,0448$ & 0,0839 \\
\hline
\end{tabular}

\begin{tabular}{|l|l|l|l|l|}
\hline \multicolumn{2}{|l|}{ Tabelle 35: Deskriptive Statistik der Als der strukturellen ROI „Wort Wernicke“ } \\
\hline & Gültige Anzahl & Median & Minimum & Maximum \\
\hline Linkshemisphärisch höhere FA Gruppe N & 13 & 1,000 & 0,193 & 1,000 \\
Linkshemisphärisch höhere FA Gruppe E & 4 & 1,000 & 0,122 & 1,000 \\
\hline Rechtshemisphärisch höhere FA Gruppe N & 6 & $-0,356$ & $-1,000$ & $-0,179$ \\
Rechtshemisphärisch höhere FA Gruppe E & 3 & $-0,261$ & $-0,481$ & $-0,125$ \\
\hline Symmetrisch verteilte FA Gruppe N & 4 & 0,0173 & $-0,0214$ & 0,0294 \\
Symmetrisch verteilte FA Gruppe E & 5 & 0,00152 & $-0,0846$ & 0,0534 \\
\hline
\end{tabular}

\begin{tabular}{|l|l|l|l|l|}
\hline \multicolumn{2}{|l|}{ Tabelle 36: Deskriptive Statistik der Als der strukturellen ROI „Satz Wernicke“ } \\
\hline & Gültige Anzahl & Median. & Minimum & Maximum \\
\hline Linkshemisphärisch höhere FA Gruppe N & 14 & 1,000 & 0,156 & 1,000 \\
Linkshemisphärisch höhere FA Gruppe E & 5 & 1,000 & 0,166 & 1,000 \\
\hline Rechtshemisphärisch höhere FA Gruppe N & 3 & $-0,182$ & $-1,000$ & $-0,165$ \\
Rechtshemisphärisch höhere FA Gruppe E & 2 & $-0,624$ & $-1,000$ & $-0,249$ \\
\hline Symmetrisch verteilte FA Gruppe N & 6 & 0,0360 & $-0,0362$ & 0,0845 \\
Symmetrisch verteilte FA Gruppe E & 5 & $-0,00679$ & $-0,0657$ & 0,0581 \\
\hline
\end{tabular}




\begin{tabular}{|l|l|l|l|}
\hline \multicolumn{2}{|l|}{ Tabelle 37: Mann-Whitney U-Test der Als für die Gruppe N gegen E } \\
\hline & $p$-Wert & Gültige Anzahl Gruppe N & Gültige Anzahl Gruppe E \\
\hline Al ROI „Wort Broca“ & 0,017479 & 23 & 12 \\
\hline Al ROI „Satz Broca“ & 0,000201 & 23 & 12 \\
\hline Al ROI "Wort Wernicke & 0,332150 & 23 & 12 \\
\hline Al ROI „Satz Wernicke“ & 0,175196 & 23 & 12 \\
\hline \multicolumn{2}{|l}{ gruppiert nach Gruppe, Test signifikant ab p <0,05000 } \\
\hline
\end{tabular}

Tabelle 38: Mann-Whitney U-Test der Als für die Gruppe N gegen E links

\begin{tabular}{|l|l|l|l|}
\hline & $p$-Wert & Gültige Anzahl Gruppe N & Gültige Anzahl Gruppe E links \\
\hline Al ROI „Wort Broca“ & 0,011911 & 23 & 9 \\
\hline Al ROI „Satz Broca“ & 0,001670 & 23 & 9 \\
\hline Al ROI „Wort Wernicke“ & 0,753257 & 23 & 9 \\
\hline Al ROI „Satz Wernicke“ & 0,304483 & 23 & 9 \\
\hline \multicolumn{2}{|l}{ gruppiert nach Gruppe, Test signifikant ab p <0,05 }
\end{tabular}

Tabelle 39: Vorzeichentest Gruppen N, E und E links; signifikant ab $p<0,05$

\begin{tabular}{|l|l|l|l|}
\hline & $p$-Werte Gruppe N & $p$-Werte Gruppe E & $p$-Werte Gruppe E links \\
\hline LI sb \& Al sb & 0,077100 & 0,148915 & 0,504985 \\
LI sw \& Al sw & 0,772830 & 0,723674 & 1,000000 \\
LI wb \& Al wb & 0,096092 & 0,227800 & 0,504985 \\
LI ww \& Al ww & 0,546494 & 0,045500 & 0,041227 \\
\hline sb = ROI „Satz Broca“; sw = ROI „Satz Wernicke“; wb = ROI „Wort Broca“; ww = ROI „Wort Wernicke“
\end{tabular}




\begin{tabular}{|c|c|c|c|c|c|c|c|c|}
\hline Proband & Gruppe & $\begin{array}{l}\text { Rekru- } \\
\text { tierung }\end{array}$ & $\begin{array}{l}\text { MRT- } \\
\text { Daten- } \\
\text { erhebung }\end{array}$ & $\begin{array}{l}\text { fMRT- } \\
\text { Datenver- } \\
\text { arbeitung }\end{array}$ & $\begin{array}{l}\text { fMRT- } \\
\text { Daten- } \\
\text { analyse }\end{array}$ & $\begin{array}{l}\text { DTI- } \\
\text { Daten- } \\
\text { analyse }\end{array}$ & $\begin{array}{l}\text { Berechnung } \\
\text { Lls und Als }\end{array}$ & $\begin{array}{l}\text { Statistische } \\
\text { Auswertung }\end{array}$ \\
\hline 1 & $\mathrm{~N}$ & Bo & Bo & Bo & Bo & Bo & $\mathrm{Zi}$ & $\mathrm{Zi}$ \\
\hline 2 & $\mathrm{~N}$ & Bo & Bo & Bo & Bo & Bo & $\mathrm{Zi}$ & $\mathrm{Zi}$ \\
\hline 3 & $\mathrm{~N}$ & Bo & Bo & Bo & Bo & Bo & $\mathrm{Zi}$ & $\mathrm{Zi}$ \\
\hline 4 & $\mathrm{~N}$ & Bo & Bo & Bo & Bo & Bo & $\mathrm{Zi}$ & $\mathrm{Zi}$ \\
\hline 5 & $\mathrm{~N}$ & Bo & Bo & Bo & Bo & Bo & $\mathrm{Zi}$ & Zi \\
\hline 6 & $\mathrm{~N}$ & Bo & Bo & Bo & Bo & Bo & $\mathrm{Zi}$ & Zi \\
\hline 7 & $\mathrm{~N}$ & Bo & Bo & Bo & Bo & Bo & $\mathrm{Zi}$ & $\mathrm{Zi}$ \\
\hline 8 & $\mathrm{~N}$ & Bo & Bo & Bo & Bo & Bo & $\mathrm{Zi}$ & $\mathrm{Zi}$ \\
\hline 9 & $\mathrm{~N}$ & Bo & Bo & Bo & Bo & Bo & $\mathrm{Zi}$ & $\mathrm{Zi}$ \\
\hline 10 & $\mathrm{~N}$ & Bo & Bo & $\mathrm{Zi}$ & $\mathrm{Zi}$ & $\mathrm{Zi}$ & $\mathrm{Zi}$ & $\mathrm{Zi}$ \\
\hline 11 & $\mathrm{~N}$ & $\mathrm{Zi}$ & Zi & $\mathrm{Zi}$ & $\mathrm{Zi}$ & $\mathrm{Zi}$ & $\mathrm{Zi}$ & Zi \\
\hline 12 & $\mathrm{~N}$ & $\mathrm{Zi}$ & $\mathrm{Zi}$ & $Z i$ & $\mathrm{Zi}$ & $Z i$ & $\mathrm{Zi}$ & $\mathrm{Zi}$ \\
\hline 13 & $\mathrm{~N}$ & $\mathrm{Zi}$ & $\mathrm{Zi}$ & $\mathrm{Zi}$ & $\mathrm{Zi}$ & Zi & $\mathrm{Zi}$ & $\mathrm{Zi}$ \\
\hline 14 & $\mathrm{~N}$ & $\mathrm{Zi}$ & $\mathrm{Zi}$ & $\mathrm{Zi}$ & $\mathrm{Zi}$ & $\mathrm{Zi}$ & $\mathrm{Zi}$ & $\mathrm{Zi}$ \\
\hline 15 & $\mathrm{~N}$ & Bo & Bo & $\mathrm{Zi}$ & $\mathrm{Zi}$ & $\mathrm{Zi}$ & $\mathrm{Zi}$ & Zi \\
\hline 16 & $\mathrm{~N}$ & Bo & Bo & Bo & Bo & Bo & $\mathrm{Zi}$ & Zi \\
\hline 17 & $\mathrm{~N}$ & $\mathrm{Zi}$ & $\mathrm{Zi}$ & Bo & $\mathrm{Zi}$ & $\mathrm{Zi}$ & $\mathrm{Zi}$ & $\mathrm{Zi}$ \\
\hline 18 & $\mathrm{~N}$ & Bo & Bo & Bo & $\mathrm{Zi}$ & Zi & $\mathrm{Zi}$ & $\mathrm{Zi}$ \\
\hline 19 & $\mathrm{~N}$ & $\mathrm{Zi}$ & $\mathrm{Zi}$ & Bo & $\mathrm{Zi}$ & $\mathrm{Zi}$ & $\mathrm{Zi}$ & $\mathrm{Zi}$ \\
\hline 20 & $\mathrm{~N}$ & $\mathrm{Zi}$ & $\mathrm{Zi}$ & Bo & $\mathrm{Zi}$ & $\mathrm{Zi}$ & $\mathrm{Zi}$ & $\mathrm{Zi}$ \\
\hline 21 & $\mathrm{~N}$ & Bo & Bo & Bo & $\mathrm{Zi}$ & Zi & $\mathrm{Zi}$ & $\mathrm{Zi}$ \\
\hline 22 & $\mathrm{~N}$ & $\mathrm{Zi}$ & $\mathrm{Zi}$ & $\mathrm{Zi}$ & $\mathrm{Zi}$ & $\mathrm{Zi}$ & $\mathrm{Zi}$ & $\mathrm{Zi}$ \\
\hline 23 & $\mathrm{~N}$ & Bo & Bo & $\mathrm{Zi}$ & $\mathrm{Zi}$ & Zi & $\mathrm{Zi}$ & $\mathrm{Zi}$ \\
\hline 24 & $\mathrm{E}$ & $Z i$ & $\mathrm{Zi}$ & $Z i$ & $Z i$ & $\mathrm{Zi}$ & $\mathrm{Zi}$ & $Z i$ \\
\hline 25 & E & $\mathrm{Zi}$ & Zi & $\mathrm{Zi}$ & Zi & $Z i$ & $\mathrm{Zi}$ & Zi \\
\hline 26 & E & $\mathrm{Zi}$ & $\mathrm{Zi}$ & $Z i$ & $\mathrm{Zi}$ & $\mathrm{Zi}$ & $\mathrm{Zi}$ & $\mathrm{Zi}$ \\
\hline 27 & E & $\mathrm{Zi}$ & $\mathrm{Zi}$ & $\mathrm{Zi}$ & $\mathrm{Zi}$ & Zi & $\mathrm{Zi}$ & $\mathrm{Zi}$ \\
\hline 28 & E & $\mathrm{Zi}$ & $\mathrm{Zi}$ & $\mathrm{Zi}$ & $\mathrm{Zi}$ & $\mathrm{Zi}$ & $\mathrm{Zi}$ & $\mathrm{Zi}$ \\
\hline 29 & $\mathrm{E}$ & $Z i$ & $Z i$ & $Z i$ & $Z i$ & $\mathrm{Zi}$ & $\mathrm{Zi}$ & $Z i$ \\
\hline 30 & E & $\mathrm{Zi}$ & Zi & $\mathrm{Zi}$ & Zi & $\mathrm{Zi}$ & $\mathrm{Zi}$ & Zi \\
\hline 31 & $\mathrm{E}$ & $\mathrm{Zi}$ & $\mathrm{Zi}$ & $\mathrm{Zi}$ & $\mathrm{Zi}$ & $\mathrm{Zi}$ & $\mathrm{Zi}$ & $\mathrm{Zi}$ \\
\hline 32 & E & $\mathrm{Zi}$ & $\mathrm{Zi}$ & $\mathrm{Zi}$ & $\mathrm{Zi}$ & Zi & $\mathrm{Zi}$ & $\mathrm{Zi}$ \\
\hline 33 & E & $\mathrm{Zi}$ & $\mathrm{Zi}$ & $\mathrm{Zi}$ & $\mathrm{Zi}$ & $\mathrm{Zi}$ & $\mathrm{Zi}$ & $\mathrm{Zi}$ \\
\hline 34 & $\mathrm{E}$ & $\mathrm{Zi}$ & $Z i$ & $Z i$ & $Z i$ & $\mathrm{Zi}$ & $\mathrm{Zi}$ & $Z i$ \\
\hline 35 & E & $\mathrm{Zi}$ & $\mathrm{Zi}$ & $\mathrm{Zi}$ & $\mathrm{Zi}$ & Zi & $\mathrm{Zi}$ & $\mathrm{Zi}$ \\
\hline
\end{tabular}




\section{LITERATURVERZEICHNIS}

Aboitiz F, Garcia VR (1997): The evolutionary origin of the language areas in the human brain. A neuroanatomical perspective. Brain Res Brain Res Rev $\underline{25}$, 381-96

Adcock JE, Wise RG, Oxbury JM, Oxbury SM, Matthews PM (2003): Quantitative fMRI assessment of the differences in lateralization of language-related brain activation in patients with temporal lobe epilepsy. Neuroimage $\underline{18}, 423-38$

Amunts K, Schleicher A, Burgel U, Mohlberg H, Uylings HB, Zilles K (1999): Broca's region revisited: cytoarchitecture and intersubject variability. J Comp Neurol $\underline{412}, 319-$ 41

Amunts K, Lenzen M, Friederici AD, Schleicher A, Morosan P, Palomero-Gallagher N, Zilles K (2010): Broca's region: novel organizational principles and multiple receptor mapping. PLoS Biol $\underline{8}$,

Assaf BA, Mohamed FB, Abou-Khaled KJ, Williams JM, Yazeji MS, Haselgrove J, Faro $\mathrm{SH}$ (2003): Diffusion tensor imaging of the hippocampal formation in temporal lobe epilepsy. AJNR Am J Neuroradiol 24, 1857-62

Avoli M, Gloor P: Epilepsy; in: Encyclopedia of neuroscience, Band 1; hrsg. v. Adelmann G; Bikäuser, Basel 1987, 400-403

Beaulieu C (2002): The basis of anisotropic water diffusion in the nervous system - a technical review. NMR Biomed $\underline{15}$, 435-55

Bell B, Hermann B, Seidenberg M, Davies K, Cariski D, Rosenbek J, Woodard A, Rutecki P, Bishop M (2002): Ipsilateral Reorganization of Language in Early-Onset Left Temporal Lobe Epilepsy. Epilepsy Behav $\underline{3}, 158-164$

Benjamini Y, Hochberg Y (1995): Controlling the False Discovery Rate: A Practical and Powerful Approach to Multiple Testing. J R Stat Soc Series B (Methodological) $\underline{57}$, 289300

Berg AT, Berkovic SF, Brodie MJ, Buchhalter J, Cross JH, van Emde Boas W, Engel J, French J, Glauser TA, Mathern GW, et al. (2010): Revised terminology and concepts for organization of seizures and epilepsies: report of the ILAE Commission on Classification and Terminology, 2005-2009. Epilepsia 51, 676-85 
Binder JR, Swanson SJ, Hammeke TA, Morris GL, Mueller WM, Fischer M, Benbadis S, Frost JA, Rao SM, Haughton VM (1996): Determination of language dominance using functional MRI: a comparison with the Wada test. Neurology $\underline{46}, 978-84$

Bonnkirch N: Sprachlateralisierung bei Patienten mit idiopathischem Stottern und bei gesunden Probanden: Ein Vergleich der Ergebnisse funktioneller Magnetresonanztomografie mit denen der Diffusionstensorbildgebung. Med. Diss. Göttingen (in Vorbereitung ${ }^{1}$ )

Bookheimer S (2002): Functional MRI of language: new approaches to understanding the cortical organization of semantic processing. Annu Rev Neurosci $\underline{25}, 151-88$

Breier JI, Castillo EM, Simos PG, Billingsley-Marshall RL, Pataraia E, Sarkari S, Wheless JW, Papanicolaou AC (2005): Atypical language representation in patients with chronic seizure disorder and achievement deficits with magnetoencephalography. Epilepsia $\underline{46}, 540-8$

Briellmann RS, Mitchell LA, Waites AB, Abbott DF, Pell GS, Saling MM, Jackson GD (2003): Correlation between language organization and diffusion tensor abnormalities in refractory partial epilepsy. Epilepsia $\underline{44}, 1541-5$

Brodmann K: Vergleichende Lokalisationslehre der Großhirnrinde in ihren Prinzipien dargestellt auf Grund des Zellenbaues. 1. Auflage; Johann Ambrosius Barth Verlag, Leipzig 1909

Concha L, Beaulieu C, Collins DL, Gross DW (2009): White-matter diffusion abnormalities in temporal-lobe epilepsy with and without mesial temporal sclerosis. J Neurol Neurosurg Psychiatry $\underline{80}$, 312-9

Cousin E, Baciu M, Pichat C, Kahane P, Le Bas JF (2008): Functional MRI evidence for language plasticity in adult epileptic patients: Preliminary results. Neuropsychiatr Dis Treat $\underline{4}, 235-46$

Demonet JF, Thierry G, Cardebat D (2005): Renewal of the neurophysiology of language: functional neuroimaging. Physiol Rev $\underline{85}$, 49-95

Detre JA (2004): fMRI: applications in epilepsy. Epilepsia 45 Suppl 4, 26-31

\footnotetext{
${ }^{1}$ Mit freundlicher Genehmigung des Verfassers.
} 
Diner HC, Weimar C: Erster epileptischer Anfall und Epilepsien im Erwachsenenalter; in: Leitlinien für Diagnostik und Therapie in der Neurologie, 5. Auflage; hrsg. v. Diener HC, Weimar C, Kommission Leitlinien der DGN; Thieme Verlag, Stuttgart 2012, 28-47

Dronkers NF, Pinker S, Damasio A: Kapitel 59: Language and the Aphasias; in: Principles of neural science, 4. Auflage; hrsg. v. Kandel ER, Schwartz JH, Jessell TM; McGraw-Hill, Health Professions Division, New York 2000, 1169 -1187

Elger CE, Speckmann EJ (1983): Penicillin-induced epileptic foci in the motor cortex: vertical inhibition. Electroencephalogr Clin Neurophysiol $\underline{56}, 604-22$

Ellmore TM, Beauchamp MS, Breier JI, Slater JD, Kalamangalam GP, O'Neill TJ, Disano MA, Tandon N (2010): Temporal lobe white matter asymmetry and language laterality in epilepsy patients. Neuroimage $\underline{49}, 2033-44$

Engel J, Jr. (2001): Mesial temporal lobe epilepsy: what have we learned? Neuroscientist $\underline{7}, 340-52$

Fisher RS, van Emde Boas W, Blume W, Elger C, Genton P, Lee P, Engel J, Jr. (2005): Epileptic seizures and epilepsy: definitions proposed by the International League Against Epilepsy (ILAE) and the International Bureau for Epilepsy (IBE). Epilepsia 46, 470-2

Friston KJ, Jezzard P, Turner R (1994): Analysis of functional MRI time-series. Hum Brain Mapp 1, 153-171

Genovese CR, Lazar NA, Nichols T (2002): Thresholding of statistical maps in functional neuroimaging using the false discovery rate. Neuroimage $\underline{15}, 870-8$

Geschwind N (1970): The organization of language and the brain. Science $\underline{170}, 940-4$

Goebel R, Esposito F, Formisano E (2006): Analysis of functional image analysis contest (FIAC) data with brainvoyager QX: From single-subject to cortically aligned group general linear model analysis and self-organizing group independent component analysis. Hum Brain Mapp 27, 392-401

Good CD, Johnsrude I, Ashburner J, Henson RN, Friston KJ, Frackowiak RS (2001): Cerebral asymmetry and the effects of sex and handedness on brain structure: a voxelbased morphometric analysis of 465 normal adult human brains. Neuroimage $14,685-$ 700 
Hauser WA, Annegers JF, Kurland LT (1991): Prevalence of epilepsy in Rochester, Minnesota: 1940-1980. Epilepsia 32, 429-45

Helmstaedter C, Kurthen M, Linke DB, Elger CE (1997): Patterns of language dominance in focal left and right hemisphere epilepsies: relation to MRI findings, EEG, sex, and age at onset of epilepsy. Brain Cogn $\underline{33}, 135-50$

Helmstaedter C, Wietzke J, Lutz MT (2009): Unique and shared validity of the "Wechsler logical memory test", the "California verbal learning test", and the "verbal learning and memory test" in patients with epilepsy. Epilepsy Res $\underline{87}$, 203-12

Hietala SO, Silfvenius H, Aasly J, Olivecrona M, Jonsson L (1990): Brain perfusion with intracarotid injection of $99 \mathrm{mTc}-\mathrm{HM}-\mathrm{PAO}$ in partial epilepsy during amobarbital testing. Eur J Nucl Med 16, 683-7

Hufschmidt A, Lücking $\mathrm{CH}$, Rauer $\mathrm{SH}$ : Neurologie compact: Leitlinine für Klinik und Praxis. 5. Auflage; Thieme Verlag, Stuttgart 2009

Jansen A, Menke R, Sommer J, Forster AF, Bruchmann S, Hempleman J, Weber B, Knecht S (2006): The assessment of hemispheric lateralization in functional MRIrobustness and reproducibility. Neuroimage $\underline{33}, 204-17$

Jenkinson M, Bannister P, Brady M, Smith S (2002): Improved optimization for the robust and accurate linear registration and motion correction of brain images. Neuroimage 17, 825-41

Kwan P, Brodie MJ (2000): Early identification of refractory epilepsy. N Engl J Med $\underline{342}, 314-9$

Kwan P, Arzimanoglou A, Berg AT, Brodie MJ, Allen Hauser W, Mathern G, Moshe SL, Perucca E, Wiebe S, French J (2010): Definition of drug resistant epilepsy: consensus proposal by the ad hoc Task Force of the ILAE Commission on Therapeutic Strategies. Epilepsia 51, 1069-77

Lancaster JL, Rainey LH, Summerlin JL, Freitas CS, Fox PT, Evans AC, Toga AW, Mazziotta JC (1997): Automated labeling of the human brain: a preliminary report on the development and evaluation of a forward-transform method. Hum Brain Mapp $\underline{5}$, 238-42

Lancaster JL, Woldorff MG, Parsons LM, Liotti M, Freitas CS, Rainey L, Kochunov PV, Nickerson D, Mikiten SA, Fox PT (2000): Automated Talairach atlas labels for functional brain mapping. Hum Brain Mapp 10, 120-31 
Le Bihan D, Breton E, Lallemand D, Grenier P, Cabanis E, Laval-Jeantet M (1986): MR imaging of intravoxel incoherent motions: application to diffusion and perfusion in neurologic disorders. Radiology 161, 401-7

Logothetis NK, Pauls J, Augath M, Trinath T, Oeltermann A (2001): Neurophysiological investigation of the basis of the fMRI signal. Nature $\underline{412}, 150-157$

Lurito JT, Dzemidzic M (2001): Determination of cerebral hemisphere language dominance with functional magnetic resonance imaging. Neuroimaging Clin N Am 11, 355-63, x

Makris N, Kennedy DN, Mclnerney S, Sorensen AG, Wang R, Caviness VS, Jr., Pandya DN (2005): Segmentation of subcomponents within the superior longitudinal fascicle in humans: a quantitative, in vivo, DT-MRI study. Cereb Cortex $\underline{15}$, 854-69

Mathern GW, Babb TL, Armstrong DL: Hippocampal Sclerosis; in: Epilepsy: A Comprehensive Textbook; hrsg. v. Engle JJ, Pedley TA; Lippincott-Raven, Philadelphia 1997, 133-155

Matsumoto H, Ajmone-Marsan C (1964): Cortical cellular phenomena in experimental epilepsy: Ictal manifestations. Exp Neurol $\underline{9}, 305-326$

May T, Pfäffin M: Epidemiologie der Epilepsien; in: Modelle zur Versorgung schwer behandelbarer Epilepsien; Abschlusspublikation zu den Modellförderungen in der prächirurgischen Epilepsiediagnostik und Epilepsiechirurgie im Rahmen des Modellprogramms der Bundesregierung zur Verbesserung der Versorgung chronisch Kranker, Band 123: Schriftenreihe des Bundesministeriums für Gesundheit; hrsg. v. Stefan H, BMG; Nomos, Baden-Baden 2000, 13-22

Mori S, van Zijl PCM (2002): Fiber tracking: principles and strategies - a technical review. NMR Biomed $\underline{15}, 468-480$

Moseley ME, Kucharczyk J, Asgari HS, Norman D (1991): Anisotropy in diffusionweighted MRI. Magn Reson Med 19, 321-6

Muller RA, Rothermel RD, Behen ME, Muzik O, Chakraborty PK, Chugani HT (1999): Language organization in patients with early and late left-hemisphere lesion: a PET study. Neuropsychologia $\underline{37}, 545-57$

Ogawa S, Lee TM, Kay AR, Tank DW (1990): Brain magnetic resonance imaging with contrast dependent on blood oxygenation. Proc Nat Acad Scie USA 7, 9868-9872 
Okazaki MM, Evenson DA, Nadler JV (1995): Hippocampal mossy fiber sprouting and synapse formation after status epilepticus in rats: visualization after retrograde transport of biocytin. J Comp Neurol $\underline{352}, 515-34$

Oldfield RC (1971): The assessment and analysis of handedness: The Edinburgh inventory. Neuropsychologia $\underline{9}, 97-113$

Pataraia E, Simos PG, Castillo EM, Billingsley-Marshall RL, McGregor AL, Breier JI, Sarkari S, Papanicolaou AC (2004): Reorganization of language-specific cortex in patients with lesions or mesial temporal epilepsy. Neurology $\underline{63}, 1825-32$

Powell HW, Parker GJ, Alexander DC, Symms MR, Boulby PA, Wheeler-Kingshott CA, Barker GJ, Noppeney U, Koepp MJ, Duncan JS (2006): Hemispheric asymmetries in language-related pathways: a combined functional MRI and tractography study. Neuroimage $\underline{32}, 388-99$

Prince DA, Wilder BJ (1967): Control mechanisms in cortical epileptogenic foci. "Surround" inhibition. Arch Neurol 16, 194-202

Rieseberg S, Merboldt KD, Kuntzel M, Frahm J (2005): Diffusion tensor imaging using partial Fourier STEAM MRI with projection onto convex subsets reconstruction. Magn Reson Med $\underline{54}$, 486-90

Rodrigo S, Oppenheim C, Chassoux F, Hodel J, de Vanssay A, Baudoin-Chial S, Devaux B, Meder JF (2008): Language lateralization in temporal lobe epilepsy using functional MRI and probabilistic tractography. Epilepsia $\underline{49}$, 1367-76

Scharfman HE, Goodman JH, Sollas AL (2000): Granule-like neurons at the hilar/CA3 border after status epilepticus and their synchrony with area CA3 pyramidal cells: functional implications of seizure-induced neurogenesis. J Neurosci $\underline{20}, 6144-58$

Skudlarski P, Constable RT, Gore JC (1999): ROC analysis of statistical methods used in functional MRI: individual subjects. Neuroimage $\underline{9}, 311-29$

Sloviter RS (1987): Decreased hippocampal inhibition and a selective loss of interneurons in experimental epilepsy. Science $\underline{235}$, 73-6

Spreer J, Arnold S, Quiske A, Wohlfarth R, Ziyeh S, Altenmuller D, Herpers M, Kassubek J, Klisch J, Steinhoff BJ, et al. (2002): Determination of hemisphere dominance for language: comparison of frontal and temporal fMRI activation with intracarotid amytal testing. Neuroradiology $\underline{44}, 467-74$ 
Springer JA, Binder JR, Hammeke TA, Swanson SJ, Frost JA, Bellgowan PS, Brewer CC, Perry HM, Morris GL, Mueller WM (1999): Language dominance in neurologically normal and epilepsy subjects: a functional MRI study. Brain 122 ( Pt 11), 2033-46

Stefan H: Epilepsien: Diagnose und Behandlung. 2. Auflage; Chapman\&Hall, Weinheim 1995

Stefan H (2000): Pre-surgical evaluation for epilepsy surgery - European standards. European Federation of Neurological Societies Task Force. Eur J Neurol $\underline{7}, 119-22$

Stippich C, Mohammed J, Kress B, Hahnel S, Gunther J, Konrad F, Sartor K (2003): Robust localization and lateralization of human language function: an optimized clinical functional magnetic resonance imaging protocol. Neurosci Lett $\underline{346}, 109-13$

Sundgren PC, Dong Q, Gomez-Hassan D, Mukherji SK, Maly P, Welsh R (2004): Diffusion tensor imaging of the brain: review of clinical applications. Neuroradiology $\underline{46}$, $339-50$

Szaflarski JP, Binder JR, Possing ET, McKiernan KA, Ward BD, Hammeke TA (2002): Language lateralization in left-handed and ambidextrous people: fMRI data. Neurology $\underline{59}, 238-44$

Talairach J, Tournoux P: Co-planar stereotaxic atlas of the human brain: 3-dimensional proportional system: an approach to cerebral imaging. 1. Auflage; Thieme Medical Publishers, New York 1988

Thivard L, Lehericy S, Krainik A, Adam C, Dormont D, Chiras J, Baulac M, Dupont S (2005): Diffusion tensor imaging in medial temporal lobe epilepsy with hippocampal sclerosis. Neuroimage $\underline{28}, 682-90$

Urbach H, Von Oertzen J, Klemm E, Koenig R, Linke DB, Kurthen M, Schramm J, Elger CE (2002): Selective middle cerebral artery Wada tests as a part of presurgical evaluation in patients with drug-resistant epilepsies. Epilepsia $\underline{43}, 1217-23$

Vernooij MW, Smits M, Wielopolski PA, Houston GC, Krestin GP, van der Lugt A (2007): Fiber density asymmetry of the arcuate fasciculus in relation to functional hemispheric language lateralization in both right- and left-handed healthy subjects: a combined fMRI and DTI study. Neuroimage $\underline{35}, 1064-76$

Wada J, Rasmussen T (1960): Intracarotid Injection of Sodium Amytal for the Lateralization of Cerebral Speech Dominance. J Neurosurg 17, 266-282 
Woermann FGM, Jokeit HP, Luerding RM, Freitag HM, Schulz RM, Guertler S, Okujava MMDP, Wolf PM, Tuxhorn IM, Ebner AM (2003): Language lateralization by Wada test and fMRI in 100 patients with epilepsy. Neurology $\underline{61}, 699-701$

Yoo SY, Chang KH, Song IC, Han MH, Kwon BJ, Lee SH, Yu IK, Chun CK (2002): Apparent diffusion coefficient value of the hippocampus in patients with hippocampal sclerosis and in healthy volunteers. AJNR Am J Neuroradiol $\underline{23}, 809-12$ 


\section{Danksagung}

Ich danke der Abteilung „Klinische Neurophysiologie“ der Universitätsmedizin Göttingen, insbesondere Herrn Prof. Dr. Frithjof Tergau und Herrn Prof. Dr. Walter Paulus, für die interessante Aufgabenstellung und die wissenschaftliche und fachliche Unterstützung.

Besonderer Dank gilt Herrn Dr. Stephan Henning für die exzellente und konstante Betreuung in allen Phasen der Dissertation. Von der Einführung in die Epileptologie über die computergestützte Datenverarbeitung und Auswertung bis hin zum sorgfältigen Korrigieren der Dissertation stand mir Dr. Stephan Henning stets mit Rat und Tat zur Seite. Ohne sein unermüdliches Engagement wäre die Fertigstellung dieser Arbeit nicht möglich gewesen.

Der Epilepsie-Ambulanz der Abteilung „Klinische Neurophysiologie“ der Universitätsmedizin Göttingen danke ich für die Unterstützung bei der Rekrutierung der Studienteilnehmer und für die fachliche Betreuung.

Darüber hinaus danke ich der Forschungsgruppe „MR-Forschung in der Neurologie und Psychiatrie" der Universitätsmedizin Göttingen für die hervorragende Zusammenarbeit bei der Datenerhebung und technischen Durchführung der Studie.

Herrn Dr. Peter Dechent, Herrn Dr. Jürgen Baudewig und Herrn Dr. Gunther Helms danke ich für fruchtbare Diskussionen.

Insbesondere danke ich Frau Pfahlert, die wertvolle Stunden ihres Wochenendes für die Durchführung von Messungen geopfert hat.

Ein besonderes Dankeschön gilt den teilnehmenden Patienten und Probanden, ohne deren Hilfe diese Arbeit nicht hätte durchgeführt werden können. 


\section{Lebenslauf}

Am 11. November 1983 wurde ich, Dorothee Zingel, in Meppen geboren.

Von 1990 bis 1994 besuchte ich die Paul-Gerhard Grundschule in Meppen. Anschließend besuchte ich von 1994 bis 1996 das Gymnasium Marianum in Meppen. Im Juli 1996 zog meine Familie nach Edewecht. Hier besuchte ich von 1996 bis 2003 das Gymnasium Bad Zwischenahn/Edewecht. Meine Schullaufbahn beendete ich im Juni 2003 erfolgreich mit dem Abitur.

Zum Wintersemester 2003/2004 begann ich das Studium der Humanmedizin an der Georg-August-Universität in Göttingen. Im September 2005 bestand ich den ersten Abschnitt der Ärztlichen Prüfung. Mein Praktisches Jahr erfolgte von August 2008 bis Juli 2009. Das chirurgische Tertial absolvierte ich im Westmead Hospital in Sydney, Australien, und im Städtischen Klinikum Lüneburg. Anschließend verbrachte ich mein Wahltertial in der Gynäkologie im Städtischen Klinikum Lüneburg. Die Innere Medizin absolvierte ich je zur Hälfte im Gilbert Bain Hospital in Shetland, Schottland, sowie im Städtischen Klinikum Lüneburg.

Im November 2009 schloss ich mein Studium mit dem zweiten Abschnitt der Ärztlichen Prüfung ab.

Als approbierte Ärztin begann ich zunächst eine basischirurgische Weiterbildung. Von Juli 2010 bis August 2011 war ich als Assistenzärztin in der Herz-, Thorax-, Transplantations- und Gefäßchirurgie an der Medizinischen Hochschule Hannover tätig.

Seit September 2011 bin ich als Assistenzärztin in der Henriettenstiftung Hannover angestellt und befinde mich aktuell in der Facharztweiterbildung Gynäkologie und Geburtshilfe. 